\title{
ON THE HOMOTOPY THEORY FOR LIE $\infty$-GROUPOIDS, WITH AN APPLICATION TO INTEGRATING $L_{\infty}$-ALGEBRAS
}

\author{
CHRISTOPHER L. ROGERS AND CHENCHANG ZHU
}

\begin{abstract}
Lie $\infty$-groupoids are simplicial Banach manifolds that satisfy an analog of the Kan condition for simplicial sets. An explicit construction of Henriques produces certain Lie $\infty$-groupoids called "Lie $\infty$-groups" by integrating finite type Lie $n$-algebras. In order to study the compatibility between this integration procedure and the homotopy theory of Lie $n$-algebras introduced in the companion paper [36], we present a homotopy theory for Lie $\infty$-groupoids. Unlike Kan simplicial sets and the higher geometric groupoids of Behrend and Getzler, Lie $\infty$-groupoids do not form a category of fibrant objects (CFO), since the category of manifolds lacks pullbacks. Instead, we show that Lie $\infty$-groupoids form an "incomplete category of fibrant objects" in which the weak equivalences correspond to "stalkwise" weak equivalences of simplicial sheaves. This homotopical structure enjoys many of the same properties as a $\mathrm{CFO}$, such as having, in the presence of functorial path objects, a convenient realization of its simplicial localization. We further prove that the acyclic fibrations are precisely the hypercovers, which implies that many of Behrend and Getzler's results also hold in this more general context. As an application, we show that Henriques' integration functor is an exact functor with respect to a class of distinguished fibrations which we call "quasi-split fibrations". Such fibrations include acyclic fibrations as well as fibrations that arise in string-like extensions. In particular, integration sends $L_{\infty}$ quasi-isomorphisms to weak equivalences, quasi-split fibrations to Kan fibrations, and preserves acyclic fibrations, as well as pullbacks of acyclic/quasi-split fibrations.
\end{abstract}

\section{Contents}

1. Introduction

1.1. Summary of main results

1.2. Outline of paper

1.3. Acknowledgments

2. Incomplete categories of fibrant objects 6

2.1. Factorization 7

2.2. Simplicial localization $\quad 10$

3. Higher groupoids and Kan fibrations $\quad 15$

3.1. Preliminaries and notation $\quad 15$

3.2. Pretopologies 16

3.3. Pretopologies for Banach manifolds 16

$\begin{array}{lll}\text { 3.4. Kan fibrations and higher groupoids in }(\mathrm{M}, \mathcal{T}) & 17\end{array}$

3.5. Representability results $\quad 18$

4. Points for categories with pretopologies 19

2000 Mathematics Subject Classification. 55U35; 18G30; 22A22; 17B55.

Key words and phrases. simplicial manifold, Lie $\infty$-groupoid, $L_{\infty}$-algebra, category of fibrant objects, hypercover. 
4.1. Points for Banach manifolds 20

4.2. Matching objects and stalkwise Kan fibrations 21

5. Stalkwise weak equivalences 24

5.1. Simplicial homotopy groups for higher groups 24

5.2. Stalkwise acyclic fibrations 25

6. Hypercovers 26

6.1. Locally stalkwise pretopologies $\quad 27$

6.2. Locally stalkwise pretopologies for Banach manifolds 28

7. Higher groupoids as an incomplete category of fibrant objects 31

7.1. Path object 31

7.2. The iCFO structure 34

7.3. Alternative characterization of weak equivalences 40

8. Lie $n$-algebras $\quad 42$

8.1. $L_{\infty}$-algebras and their morphisms $\quad 42$

8.2. Finite type Lie $n$-algebras $\quad 43$

8.3. Fibrations of Lie $n$-algebras $\quad 45$

8.4. Postnikov tower for Lie $n$-algebras 46

8.5. Quasi-split fibrations and decomposition of towers 47

8.6. Maurer-Cartan elements 49

9. Integration of Lie $n$-algebras $\quad 51$

9.1. From Lie $n$-algebras to Lie $\infty$-groups $\quad 51$

9.2. Integrating fibrations $\quad 52$

9.3. Integrating weak equivalences 60

9.4. The exactness of integration 62

Appendix A. Proof of Lemma 7.14 63

Appendix B. Sheaves on large categories $\quad 66$

B.1. Grothendieck universes 66

B.2. Sheaves on locally $\mathcal{U}$-small categories 66

B.3. Independence of choice of ambient universe 68

$\begin{array}{ll}\text { References } & 69\end{array}$

\section{INTRODUCTION}

Lie $\infty$-groupoids, introduced by Henriques [17], are simplicial Banach manifolds that satisfy a certain diffeo-geometric analog of the "horn filling" condition for Kan simplicial sets. A Lie $\infty$-groupoid for which all horns of dimension $>n$ are filled uniquely is called a "Lie $n$-groupoid". A Lie 0 -groupoid is a just a Banach manifold, while a Lie 1-groupoid is the nerve of a Lie groupoid. In general, Lie $n$-groupoids serve as models for differentiable $n$-stacks.

Important examples of Lie $n$-groupoids are "Lie $n$-groups". These are Lie $n$ groupoids that have a single 0 -simplex (i.e. reduced Lie $n$-groupoids). Lie $n$-groups have been used to construct diffeo-geometric models for the higher stages of the Whitehead tower of the orthogonal group. The most famous of these is the "String Lie 2-group": its geometric realization is a topological group whose homotopy type is the 3-connected cover of the orthogonal group. Initial interest in the String 2group stemmed from its appearance in string theory and possible applications to 
geometric models of elliptic cohomology. (See Sec. 1.2 of [17] for a summary and also Sec. 7 of [42].)

With these applications in mind, Henriques developed a smooth analog of Sullivan's realization functor from rational homotopy theory which produces Lie $n$ groups by "integrating" finite type Lie $n$-algebras. Lie $n$-algebras are non-negatively graded chain complexes concentrated in the first $n-1$ degrees, equipped with a collection of multi-linear brackets which satisfy a coherent homotopy analog of the Jacobi identity for differential graded Lie algebras. (These are also known as $n$-term $L_{\infty}$-algebras, or $n$-term homotopy Lie algebras $[6,24]$.) A Lie 1-algebra is just a Lie algebra.

Finite type Lie $n$-algebras have a good notion of a homotopy theory which can be modeled in a variety of ways $[9,36,39]$. In these contexts, morphisms between Lie $n$-algebras are significantly "weaker" than just linear maps which preserve the brackets. But every morphism between Lie $n$-algebras induces a chain map between the underlying complexes. A morphism between Lie $n$-algebras is a weak equivalence when the induced chain map gives an isomorphism on the corresponding homology groups. (Such morphisms are also known as " $L_{\infty}$-quasi-isomorphisms".)

What is still missing from this story is a full understanding of the relationship between the homotopy theory of Lie $n$-algebras and the homotopy theory of Lie $n$ groups. The situation is understood in some special cases, for example, for strict Lie 2-algebras and Lie 2-groups [31]. But in general, one would hope that Henriques' integration functor would send a weak equivalence between Lie $n$-algebras to a weak equivalence between Lie $n$-groups.

It turns out that presenting a user-friendly homotopy theory for Lie $n$-groupoids is a bit subtle. This is mostly due to the fact that the category of Banach manifolds lacks several desirable properties, such as the existence of pullbacks. Recently, Behrend and Getzler [3] showed that higher groupoids internal to certain geometric contexts called "descent categories" form a category of fibrant objects (CFO) for a homotopy theory, in the sense of Brown [5]. Roughly, a descent category is a category of "spaces" which has all finite limits equipped with a distinguished class of morphisms called "covers" satisfying some axioms. Examples include the category of schemes with surjective étale morphisms as covers, as well as the category of Banach analytic spaces, with surjective submersions as covers. The fibrations in the Behrend-Getzler CFO structure for $n$-groupoids are natural generalizations of Kan fibrations, while the acyclic fibrations are precisely the so-called "hypercovers". As Behrend and Getzler show, this data completely determines the weak equivalences between geometric $n$-groupoids via a very nice combinatorial characterization [3, Thm. 5.1].

Unfortunately, the category of Banach manifolds - regardless of the choice of covers - does not form a descent category, since it lacks finite limits. Hence, Behrend and Getzler's results do not apply directly in this context. (Nor, unfortunately, does the related work of Pridham [32].) It is worth emphasizing that finite-dimensional Lie $n$-groupoids form a full subcategory of the category of $n$-groupoids internal to the category of $C^{\infty}$-schemes (in the sense of Dubuc [10]). The latter category, as Behrend and Getzler note, is a descent category. Kan fibrations between Lie $n$-groupoids first appeared in the work of Henriques [17], while hypercovers for Lie $n$-groupoids are featured prominently in the work of the second author [43] and Wolfson [42]. In particular, the second author defines two Lie $n$-groupoids to be 
"Morita equivalent" if they are connected by a span of hypercovers. Wolfson's work on $n$-bundles is also quite relevant here: he generalizes aspects of Behrend and Getzler's machinery to the context of Lie $n$-groupoids, but he did not require an explicit presentation of their homotopy theory.

1.1. Summary of main results. In Theorem 7.1, we show that Lie $n$-groupoids form what we call - for lack of better terminology - an "incomplete category of fibrant objects" (iCFO) for a homotopy theory. Just like in a category of fibrant objects, an iCFO is equipped with two important classes of morphisms: weak equivalences and fibrations, and as usual, the morphisms which lie in the intersection of the two are called acyclic fibrations. The axioms of an iCFO (Def. 2.1) are identical to those of a CFO, except we do not assume the existence of pullbackseven along fibrations. We define the weak equivalences of Lie $n$-groupoids to be those smooth simplicial morphisms which correspond, via the Yoneda embedding, to "stalkwise weak equivalences" between the associated simplicial sheaves. Stalkwise weak equivalences (Def. 5.1) are a natural choice for weak equivalences between simplicial sheaves, appearing in the work of Brown [5], and specifically in diffeogeometric contexts in the work of Dugger [11], Nikolaus, Schreiber, and Stevenson [29], and Freed and Hopkins [14]

The fibrations in our iCFO structure for Lie $n$-groupoids are the "Kan fibrations" (Def. 3.3) introduced by Henriques. The acyclic fibrations are, by definition, those Kan fibrations which are also stalkwise weak equivalences. However, we show in Prop. 6.7 and Prop. 6.12 that any morphism which is both a Kan fibration and a stalkwise weak equivalence is a hypercover (Def. 6.1). This is because the category of Banach manifolds can be given the structure of what we call a "locally stalkwise pretopology" (Def. 6.5). This is a diffeo-geometric result, and relies on the fact that the inverse function theorem holds for Banach manifolds. And so the acyclic fibrations in our iCFO structure are precisely the hypercovers, just like in the work of Behrend and Getzler. Hence, using their results, we demonstrate in Sec. 7.3 that the weak equivalences between Lie $n$-groupoids can be characterized completely by combinatorial data that only involves maps between Banach manifolds. No reference to simplicial sheaf theory is actually needed.

One advantage of defining the weak equivalences to be stalkwise weak equivalences is that it allows us to connect to the homotopy theory of Lie $n$-algebras, via Henriques' integration functor, in a straightforward way. We can give a rather concise proof that the integration of a $L_{\infty}$ quasi-isomorphism is a stalkwise weak equivalence (Thm. 9.15).

This is, in fact, only a part of a much stronger result. The first author has shown in a companion paper [36] that the category of finite type Lie $n$-algebras admits a CFO structure that, roughly speaking, lifts the projective model structure on non-negatively graded chain complexes. In particular, the weak equivalences are precisely the $L_{\infty}$ quasi-isomorphisms, and the fibrations are those $L_{\infty}$-morphisms whose linear term is surjective in all positive degrees. We summarize this result in Thm. 8.3 of the present paper. Furthermore, in Remark 8.4, we use some preliminary results concerning the differentiation of Lie $\infty$-groups to explain why this notion of fibration between Lie $n$-algebras is the "correct" one for our applications.

It turns out that not every fibration of Lie $n$-algebras integrates to a Kan fibration (Remark 9.11). So the integration functor is not an exact functor, in the usual sense of homotopical algebra. However, in Def. 2.8, we introduce the notion of a functor 
being exact with respect to a class of distinguished fibrations. This is a mild and controllable generalization, and it provides us with a useful way to compare iCFOs.

Indeed, Thm. 9.16 demonstrates that integration is compatible with a substantial amount of the iCFO structure. More precisely, we show that Henriques' integration functor is exact with respect to the class of so-called "quasi-split fibrations". This class of fibrations includes all acyclic fibrations, as well as the fibrations which naturally arise in the construction of the string Lie 2-algebra. We interpret this result as step one of a larger project in progress whose goal is to prove an analog of "Lie's Second Theorem" for Lie $n$-groups and Lie $n$-algebras.

1.2. Outline of paper. Throughout, we try to write for a somewhat broader audience, which could include, for example, readers from differential/Poisson geometry with interests in both Lie groupoid theory and $L_{\infty}$-algebras. We attempt a selfcontained presentation, within reason, and use only a minimal amount of technical machinery. We recognize that some of the auxiliary results presented here can reside in a more general framework well known to experts in abstract homotopy theory.

We begin in Section 2, where we give the axioms for an incomplete category of fibrant objects (iCFO) for a homotopy theory. We show that many of the nice properties which hold for CFOs also hold for iCFOs. For example, we show that the mapping space between two objects in the Dwyer-Kan simplicial localization of a small iCFO, equipped with functorial path objects and functorial pullbacks of acyclic fibrations, can be described as the nerve of a category of spans. We also introduce in this section the notion of an functor being exact with respect to a class of distinguished fibrations. Such a functor has many of the same properties as an exact functor between CFOs. The main difference is that the functor is only required to preserve those fibrations contained in a distinguished subclass of fibrations in the source category.

In Section 3, we recall the definitions of an $n$-groupoid object and a Kan fibration in a large category (such as the category of Banach manifolds) equipped with a "pretopology". Many of the basic constructions in this section and throughout the paper require taking limits in this category which a priori do not exist. Hence, limits must be treated as limits of sheaves, and then shown to be representable. Furthermore, we later on define weak equivalences between $n$-groupoids in terms of the simplicial Yoneda embedding. We therefore need to deal with sheaves over large categories. To resolve any set-theoretical problems, we employ the standard workaround by passing to a larger Grothendieck universe. In theory, this could introduce a dependence on this "enlargement" (e.g., see [40]). So we show explicitly in Appendix B that, for the case of Lie $n$-groupoids, all of our results are independent of choice of Grothendieck universe.

In Section 4, we recall the notion of "points" for categories of sheaves, which generalize the notion of stalks. In particular, we consider a collection of points for sheaves over the category of Banach manifolds, which generalizes those found in the literature (e.g., [11, 29] ) for finite-dimensional manifolds. We also collect in this section some useful results regarding matching objects and various notions of epimorphisms and surjections for pretopologies equipped with a collection of points. We use these notions in Section 5 to define stalkwise weak equivalences between $n$-groupoids. We also show in this section that any morphism between $n$ groups (reduced $n$-groupoids) which induces an isomorphism of the corresponding 
simplicial homotopy group sheaves (in the sense of Joyal [21] and Henriques [17]) is a stalkwise weak equivalence. We use this result in Section 9 where we consider the integration $L_{\infty}$ quasi-isomorphisms.

The definition of hypercover is recalled in Section 6, and we introduce the notion of a category equipped with a "locally stalkwise pretopology". We show that the category of Banach manifolds equipped with the surjective submersion pretopology is an example. In Section 7 , we prove $\infty$-groupoids in a category equipped with a locally stalkwise pretopology form an iCFO. We note that this proof can be easily refined to show that $n$-groupoids for finite $n$ also have an iCFO structure. We also demonstrate in this section that the weak equivalences for this iCFO structure can be described without the need of simplicial sheaves, in analogy with a result of Behrend and Getzler.

In Section 8, we recall from [36] the CFO structure on the category of finite type Lie $n$-algebras. We also summarize a number of technical results from [36] concerning: the "strictification" of fibrations between Lie $n$-algebras, the properties of Maurer-Cartan sets, and the decomposition of Postnikov towers. We also introduce the notion of a quasi-split fibration between Lie $n$-algebras, and we provide some motivating examples.

Finally, in Section 9 we extend Henriques' results on the integration of Postnikov towers of Lie $n$-algebras. We use these to show that the integration of a quasi-split fibration is a Kan fibration, and that the integration of a $L_{\infty}$ quasi-isomorphism is a stalkwise weak equivalence. We conclude with Theorem 9.16, which is our main result: The integration functor is exact with respect to the class of quasi-split fibrations.

1.3. Acknowledgments. The authors gratefully thank Ezra Getzler for many helpful conversations, and especially for sharing an early version of his preprint with Kai Behrend. CLR thanks Dave Carchedi for helpful discussions. The authors also wish to thank an anonymous referee for their suggestions on improving the exposition and strengthening the results of this work.

Parts of this paper were written during CLR's stay at the Mathematical Research Institute of the University of Melbourne (MATRIX). He thanks the institute and the organizers of the 2016 Higher Structures in Geometry and Physics program for their hospitality and support. CLR acknowledges support by an AMS-Simons Travel Grant, and both authors acknowledge support by the DFG Individual Grant (ZH 274/1-1) "Homotopy Lie Theory".

\section{INCOMPLETE CATEGORIES OF FIBRANT OBJECTS}

In this section, we introduce a slight generalization of Brown's definition for a category of fibrant objects (CFO) for a homotopy theory [5, Sec. 1]. In particular, we do not assume the existence of certain limits in the underlying category, hence the term "incomplete". This is reasonable given our applications to $\infty$-groupoid objects in diffeo-geometric categories. We note that other variations on weakening Brown's axioms have already appeared in the literature, for example Horel's "partial Brown categories" [18]. (See Ex. 2.10 below.)

Definition 2.1. Let $\mathrm{C}$ be a category with finite products and terminal object $* \in \mathrm{C}$ equipped with two classes of morphisms called weak equivalences and fibrations. 
A morphism which is both a weak equivalence and a fibration is called an acyclic fibration. We say $\mathrm{C}$ is an incomplete category of fibrant objects (iCFO) iff:

(1) Every isomorphism in $\mathrm{C}$ is an acyclic fibration.

(2) The class of weak equivalences satisfies " 2 out of 3 ". That is, if $f$ and $g$ are composable morphisms in $\mathrm{C}$ and any two of $f, g, g \circ f$ are weak equivalences, then so is the third.

(3) The composition of two fibrations is a fibration.

(4) If the pullback of a fibration exists, then it is a fibration. That is, if $Y \stackrel{g}{\rightarrow}$ $Z \stackrel{f}{\leftarrow} X$ is a diagram in $\mathrm{C}$ with $f$ a fibration, and if $X \times_{Z} Y$ exists, then the induced projection $X \times_{Z} Y \rightarrow Y$ is a fibration.

(5) The pullback of an acyclic fibration exists, and is an acyclic fibration. That is, if $Y \stackrel{g}{\rightarrow} Z \stackrel{f}{\leftarrow} X$ is a diagram in $C$ with $f$ an acyclic fibration, then the pullback $X \times_{Z} Y$ exists, and the induced projection $X \times_{Z} Y \rightarrow Y$ is an acyclic fibration.

(6) For any object $X \in \mathrm{C}$ there exists a (not necessarily functorial) path object, that is, an object $X^{I}$ equipped with morphisms

$$
X \stackrel{s}{\rightarrow} X^{I} \stackrel{\left(d_{0}, d_{1}\right)}{\longrightarrow} X \times X
$$

such that $s$ is a weak equivalence, $\left(d_{0}, d_{1}\right)$ is a fibration, and their composite is the diagonal map.

(7) All objects of $\mathrm{C}$ are fibrant. That is, for any $X \in \mathrm{C}$ the unique map $X \rightarrow *$ is a fibration.

Remark 2.2. The only difference between the above and the original definition of Brown is axiom (4). Brown requires that the pullback of a fibration always exists.

2.1. Factorization. An important feature of a category of fibrant objects is Brown's factorization lemma in [5, Sec. 1]. The factorization lemma also holds for any iCFO.

Let $Y^{I}$ be a path object for an object $Y$ in an iCFO C. Since $Y \rightarrow *$ is a fibration, Def. 2.1 implies that the projections $\pi_{i}: Y \times Y \rightarrow Y$ are fibrations. Hence, the morphisms $d_{i}: Y^{I} \rightarrow Y$ are also fibrations. Moreover, since $d_{i} s=\operatorname{id}_{Y}$, and $s$ is a weak equivalence, the maps $d_{i}$ are acyclic fibrations. Thus we have proven

Lemma 2.3. The projection $d_{i}: Y^{I} \rightarrow Y$ is an acyclic fibration for $i=0,1$.

The proof of the next lemma is identical to the analogous lemma for categories of fibrant objects. Indeed, the proof does not require the existence of pullbacks along arbitrary fibrations.

Lemma 2.4. If $\mathrm{C}$ is an iCFO and $f: X \rightarrow Y$ is a morphism in $\mathrm{C}$, then $f$ can be factored as $f=p \circ i$, where $p$ is a fibration, and $i$ is a weak equivalence which is a section (right inverse) of an acyclic fibration.

Proof. Let $Y^{I}$ be a path object for $Y$. Lemma 2.3 implies that the pullback $X \times_{Y} Y^{I}$ of the diagram

$$
X \stackrel{f}{\rightarrow} Y \stackrel{d_{0}}{\longleftarrow} Y^{I}
$$


exists, and hence the projection $\operatorname{pr}_{1}: X \times_{Y} Y^{I} \rightarrow X$ is an acyclic fibration. Combining this fact with the commutative diagram

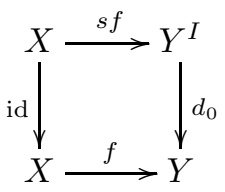

implies that $\mathrm{pr}_{1}$ has a right inverse

$$
i: X \rightarrow X \times_{Y} Y^{I}
$$

which is necessarily a weak equivalence. Moreover, if $p: X \times_{Y} Y^{I} \rightarrow Y$ is the composition

$$
X \times_{Y} Y^{I} \stackrel{\mathrm{pr}_{2}}{\longrightarrow} Y^{I} \stackrel{d_{1}}{\longrightarrow} Y
$$

then

$$
f=p \circ i \text {. }
$$

To show that $p$ is a fibration, we observe that $X \times_{Y} Y^{I}$ is also the pullback of the diagram

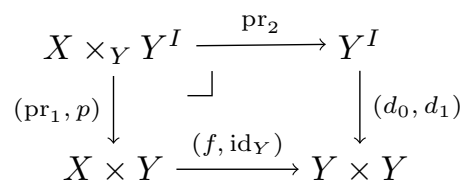

Since $\left(d_{0}, d_{1}\right)$ is a fibration, $\left(p r_{1}, p\right)$ is a fibration. Since the projection $X \times Y \rightarrow Y$ is a fibration (indeed $Y$ is fibrant), it follows that $p$ is also a fibration.

One simple fact which follows from the factorization lemma is that every weak equivalence in an iCFO yields a span of acyclic fibrations. Also, just like in the case with a CFO [3, Lemma 1.3], the weak equivalences in an iCFO are determined by the acyclic fibrations.

\section{Proposition 2.5.}

(1) If $f: X \rightarrow Y$ is a weak equivalence in an iCFO then there exists a span of acyclic fibrations $X \leftarrow Z \rightarrow Y$.

(2) A morphism $f: X \rightarrow Y$ in an iCFO is a weak equivalence if and only if it factors as $f=p \circ i$, where $p$ is an acyclic fibration, and $i$ is a section of an acyclic fibration.

Proof. If $f$ is a weak equivalence, then factor $f=p \circ i$ as in Lemma 2.4. Since $f$ and $i$ are weak equivalences, $p$ is an acyclic fibration. Hence, the legs of the span

$$
X \stackrel{\mathrm{pr}_{1}}{\longleftarrow} X \times_{Y} Y^{I} \stackrel{p}{\rightarrow} Y
$$

are acyclic fibrations.

Remark 2.6. Spans of acyclic fibrations are analogous to the notion of Morita equivalence between Lie groupoids. Indeed, in [43, Def. 2.12], two Lie $n$-groupoids are considered "Morita equivalent" iff they are connected by a span of maps called hypercovers, which we consider in Sec. 6. We will see in Sec. 7 that hypercovers are the acyclic fibrations in the iCFO structure for Lie $n$-groupoids. 
Next, we provide a working definition for the notion of an exact functor between iCFOs, which is well suited for the applications we have in mind.

Definition 2.7. Let $\mathrm{C}$ be an $\mathrm{iCFO}$ and denote by $\mathrm{fib}(\mathrm{C})$ the class of fibrations of C. A class of fibrations $S \subseteq \mathrm{fib}(\mathrm{C})$ is distinguished iff $S$ contains all acyclic fibrations and all morphisms into the terminal object of $C$.

Every iCFO has two obvious classes of distinguished fibrations: the class of all fibrations $S_{\max }=\mathrm{fib}(\mathrm{C})$, and the class $S_{\min }$ which only contains the acyclic fibrations and the morphisms into the terminal object.

Definition 2.8. Let $\mathrm{C}$ be an $\mathrm{iCFO}$ and $S$ a subclass of distinguished fibrations of C. A functor $F: C \rightarrow C^{\prime}$ between iCFOs is a (left) exact functor with respect to $S$ iff

(1) $F$ preserves the terminal object and acyclic fibrations,

(2) $F$ maps every fibration in $S$ to a fibration in $\mathrm{C}^{\prime}$, and

(3) any pullback square in $C$ of the form

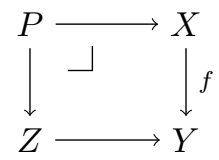

with $f$ a fibration in $S$, is mapped by $F$ to a pullback square in $C^{\prime}$.

Before we give a few examples of such functors, we note the following simple lemma:

Lemma 2.9. Let $F: C \rightarrow C^{\prime}$ be an exact functor with respect to a class $S$. Then $F$ preserves finite products and weak equivalences.

Proof. It follows from Def. 2.7 and axiom 3 above that $F$ preserves finite products. If $f: X \rightarrow Y$ is a weak equivalence in C, then Lemma 2.4 implies that $f=p \circ i$, where $p$ is an acyclic fibration and $i$ is a right inverse of an acyclic fibration. Since $F$ preserves acyclic fibrations, it follows that $F(f)$ is a weak equivalence.

Example 2.10.

(1) Let $F: C \rightarrow C^{\prime}$ be a functor between categories of fibrant objects (CFOs). Then $F$ is an exact functor between CFOs in the usual sense (Def. 2.3.3 [7]) if and only if $F$ is exact with respect to the class $S_{\max }$.

(2) An iCFO equipped with a functorial path object (see Sec. 2.2.1) has the structure of a "partial Brown category" (PBC), a notion introduced by Horel in [18, Rmk. 2.4, Prop. 2.6]. Following Horel's definition of an exact functor between PBCs [18, Def. 2.5], we say a functor between iCFOs is partially exact iff it sends weak equivalences to weak equivalences, acyclic fibrations to acyclic fibrations, and pullbacks of acyclic fibrations to pullbacks. In contrast with the first example, a product preserving functor $F: C \rightarrow C^{\prime}$ between iCFOs is partially exact if and only if $F$ is exact with respect to the class $S_{\min }$.

(3) In our main application, Thm. 9.16, we show that the integration functor from finite type Lie $n$-algebras to Lie $\infty$-groupoids is exact with respect to a class of distinguished fibrations called "quasi-split fibrations" (Def. 8.6), which lies properly between $S_{\min }$ and $S_{\max }$. 
2.2. Simplicial localization. In this section, we show that in certain cases the simplicial localization (or underlying $\infty$-category) of a small incomplete category of fibrant objects has a simple description in terms of the nerve of a category of spans. An example is a small category of $\infty$-groupoids equipped with the iCFO structure described in Sec. 7. In particular, the simplicial localization of the category of Lie $n$-groupoids is discussed in Sec. 7.2.1, and this will be useful for our future work concerning the higher category theory of Lie $\infty$-groupoids.

Recall that for any small category $\mathrm{C}$ and a wide subcategory $\mathrm{W}$ of weak equivalences, one may associate to it a simplicial category $L_{\mathrm{W}} \mathrm{C}$ (i.e., a category enriched in simplicial sets) via the Dwyer-Kan simplicial localization, or "hammock localization" [13]. The simplicial category $L_{\mathrm{W}} C$ has the same objects as $C$ and is universal with respect to the property that weak equivalences in $W$ are homotopy equivalences in $L_{\mathrm{W}} \mathrm{C}$. In particular, $\pi_{0} L_{\mathrm{W}} \mathrm{C}$, the category whose objects are those of C, and whose hom-sets are

$$
\pi_{0}\left(\operatorname{Map}_{L_{\mathrm{w}} \mathrm{C}}(X, Y)\right), \quad \forall X, Y \in \mathrm{C},
$$

is equivalent to the usual localization $\mathrm{C}\left[\mathrm{W}^{-1}\right]$, the category obtained by formally inverting the morphisms in $\mathrm{W}$.

The mapping space $\operatorname{Map}_{L_{W} C}(X, Y)$ is the direct limit of nerves of categories [13, Prop. 5.5]. Dwyer and Kan showed that when $C$ is a model category with functorial factorizations, $\operatorname{Map}_{L_{\mathrm{W}} \mathrm{C}}(X, Y)$ can be described more simply, up to weak homotopy equivalence, as the nerve of a single category of spans (e.g., Prop. 8.2 in [13] and subsequent corollaries). Weiss [41] later showed that Dwyer and Kan's proof extends to the case when $C$ is a Waldhausen category (i.e. a category with cofibrations and weak equivalences) equipped with both a cylinder functor, and canonical pushouts of cofibrations. Roughly speaking, the "opposite" of Weiss's argument is spelled out in detail for CFOs in the work of Nikolaus, Schreiber, and Stevenson [29, Thm. 3.61]. As we show below, an analogous result holds for iCFOs.

2.2.1. Functorial path objects and pullbacks. In this section, weak equivalences and fibrations will be denoted by $X \stackrel{\sim}{\rightarrow} Y$ and $X \rightarrow Y$, respectively. Furthermore, in this section, $\mathrm{C}$ will denote a small incomplete category of fibrant objects equipped with:

- functorial path objects: An assignment of a path object $X^{I}$ to each object $X \in \mathrm{C}$ and to each $f: X \rightarrow Y$, a morphism $f^{I}: X^{I} \rightarrow Y^{I}$ such that the following diagram commutes:

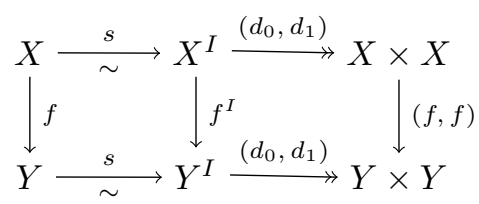

- functorial pullbacks of acyclic fibrations: An assignment to each diagram of the form $X \stackrel{f}{\rightarrow} Y \stackrel{g}{\leftarrow} Z$, in which $g$ is an acyclic fibration, a universal cone $X \stackrel{\tilde{g}}{\leftarrow} X \times_{Y} Z \stackrel{\tilde{f}}{\rightarrow} Z$ (which exists via the iCFO axioms, and in which $\tilde{g}$ is necessarily an acyclic fibration). The universal property 
implies that to each commutative diagram:

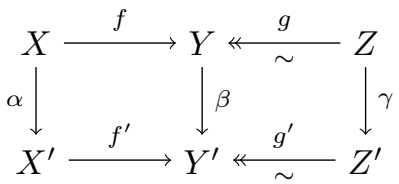

in which the right horizontal morphisms are acyclic fibrations, we obtain a unique commutative diagram

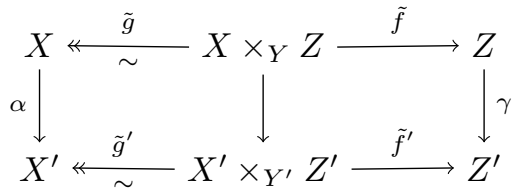

in which the left horizontal morphisms are acyclic fibrations.

Functorial path objects and pullbacks as above provide an iCFO with functorial factorizations in the sense of [34, Def. 12.1.1].

Proposition 2.11. Let $\mathrm{C}$ be an $i C F O$ with functorial path objects and functorial pullbacks of acyclic fibrations. Then each morphism $f: X \rightarrow Y$ in $\mathrm{C}$ can be canonically factored as

$$
X \stackrel{i_{f}}{\longrightarrow} X \times_{Y} Y^{I} \stackrel{p_{f}}{\longrightarrow} Y
$$

where $p_{f}$ is a fibration and $i_{f}$ is a right inverse of an acyclic fibration. Moreover, this factorization is natural: Given a commutative diagram

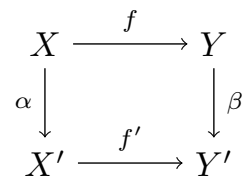

there exists a unique morphism $\gamma: X \times_{Y} Y^{I} \rightarrow X^{\prime} \times_{Y^{\prime}}{Y^{\prime}}^{I}$ such that the diagram

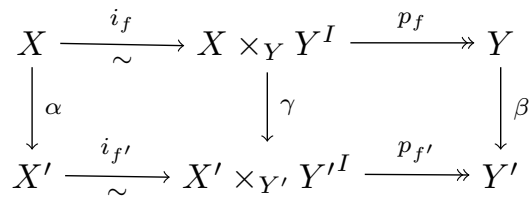

commutes.

Proof. One simply repeats the proof of the factorization lemma for an iCFO (Lemma 2.4) using the functorial path objects and pullbacks.

2.2.2. Categories of spans in $\mathrm{C}$. Denote by $\mathrm{W}_{\mathrm{f}} \subseteq \mathrm{W} \subseteq \mathrm{C}$ the subcategories of $\mathrm{C}$ consisting of acyclic fibrations and weak equivalences, respectively. For each pair of objects $X, Y \in \mathrm{C}$ we denote by $\mathrm{CW}_{f}^{-1}(X, Y)$ (respectively, $\mathrm{CW}^{-1}(X, Y)$ ) the category whose objects are spans in $C$ of the form

$$
X \leftarrow C \rightarrow Y
$$


in which the left arrow is an acyclic fibration (respectively, weak equivalence), and whose morphisms are commutative diagrams of the form

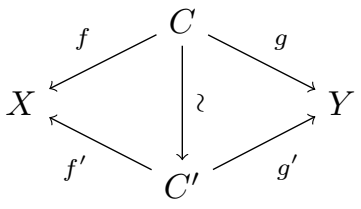

in which the vertical arrow is a weak equivalence.

We will also need generalizations of the above categories of spans. In what follows, we use Dwyer and Kan's notation for "hammock graphs" [13, Sec. 5.1]. Let $\mathbf{w}$ be a word of length $n \geq 1$ consisting of letters $\left\{C, W, W^{-1}, \mathrm{~W}_{\mathrm{f}}^{-1}\right\}$. For each pair of objects $X, Y \in \mathrm{C}$ we denote by $\mathbf{w}(X, Y)$ the category whose objects are diagrams in $\mathrm{C}$ of the form

$$
X \stackrel{f_{0}}{\longrightarrow} C_{1} \stackrel{f_{1}}{\longrightarrow} C_{2} \stackrel{f_{2}}{\longrightarrow} \cdots \frac{f_{n-2}}{2} C_{n-1} \stackrel{f_{n-1}}{=} Y
$$

in which the morphism $f_{i}$ goes to the right and is in C (resp. W) iff the $(n-i)$ th letter in $\mathbf{w}$ is $\mathbf{C}$ (resp. $\mathbf{W}$ ). Otherwise, the morphism $f_{i}$ goes to the left and is in $\mathbf{W}$ $\left(\right.$ resp. $\left.\mathrm{W}_{\mathrm{f}}\right)$ iff the $(n-i)$ th letter in $\mathbf{w}$ is $\mathbf{W}^{-1}\left(\right.$ resp. $\left.\mathbf{W}_{\mathrm{f}}^{-1}\right)$. Morphisms in $\mathbf{w}(X, Y)$ are commuting diagrams in which all vertical arrows are weak equivalences.

Proposition 2.12. Let $X, Y$ be objects of $\mathrm{C}$. The inclusion functors

$$
\begin{gathered}
\mathrm{CW}_{\mathrm{f}}^{-1}(X, Y) \stackrel{\iota}{\hookrightarrow} \mathrm{CW}^{-1}(X, Y) \\
\mathrm{WW}_{\mathrm{f}}^{-1}(X, Y) \stackrel{\iota}{\hookrightarrow} \mathrm{WW}^{-1}(X, Y)
\end{gathered}
$$

induce simplicial homotopy equivalences between the corresponding nerves

$$
N \mathrm{NW}_{\mathrm{f}}^{-1}(X, Y) \stackrel{\simeq}{\rightarrow} N \mathrm{CW}^{-1}(X, Y), \quad N \mathrm{WW}_{\mathrm{f}}^{-1}(X, Y) \stackrel{\simeq}{\rightarrow} N \mathrm{WW}^{-1}(X, Y)
$$

Proof. We use the fact that a natural transformation between functors induces a homotopy between the corresponding simplicial maps between nerves. Denote by $F: \mathrm{CW}^{-1}(X, Y) \rightarrow \mathrm{CW}_{\mathrm{f}}^{-1}(X, Y)$ the functor which assigns to a span of the form

$$
X \stackrel{f}{\sim} C \stackrel{g}{\longleftarrow} Y
$$

an object in $\mathrm{CW}_{\mathrm{f}}^{-1}(X, Y)$ via the following. First, apply the functorial factorization (Prop. 2.11) to the morphism $(f, g): C \rightarrow X \times Y$ to obtain

$$
C \stackrel{i}{\sim} C^{\prime} \stackrel{p}{\longrightarrow} X \times Y
$$

Then composing the fibration $p$ with the projections gives a span of fibrations:

$$
X \stackrel{f^{\prime}}{\longleftarrow} C^{\prime} \stackrel{g^{\prime}}{\longrightarrow} Y
$$


along with a commutative diagram

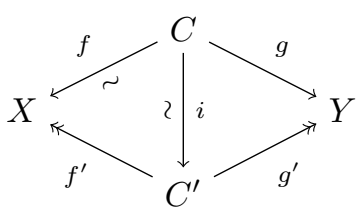

which, combined with the " 2 out of 3 " axiom implies that $f^{\prime}$ is an acyclic fibration. Hence, the diagram (2.1) is an object of $\mathrm{CW}_{\mathrm{f}}^{-1}(X, Y)$. It is easy to see that this assignment is indeed functorial, due to the use of functorial factorizations. Moreover, the weak equivalence $i$ in the diagram (2.2) gives natural transformations

$$
\operatorname{id}_{\mathrm{CW}-1(X, Y)} \rightarrow \iota \circ F, \quad \operatorname{id}_{\mathrm{CW}_{\mathrm{f}}-1(X, Y)} \rightarrow F \circ \iota
$$

Hence, $N \iota: N \mathrm{CW}_{\mathrm{f}}^{-1}(X, Y) \rightarrow N \mathrm{CW}^{-1}(X, Y)$ is a homotopy equivalence. To show $N \mathrm{WW}_{\mathrm{f}}^{-1}(X, Y) \stackrel{\simeq}{\rightarrow} N \mathrm{WW}^{-1}(X, Y)$ is a homotopy equivalence, we observe that the restriction of the functor $F$ to the subcategory $\mathrm{WW}^{-1}(X, Y)$ has as its target $\mathrm{WW}_{\mathrm{f}}^{-1}(X, Y)$, thanks to the commutative diagram $(2.2)$ and the "2 out of 3 " axiom.

2.2.3. C admits a homotopy calculus of right fractions. In the terminology of $[13$, Sec. 5.1], the $k$-simplices in $N C W^{-1}(X, Y)$ are hammocks between $X$ and $Y$ of width $k$ and type $\mathrm{CW}^{-1}$. There is simplicial map (i.e., the reduction map)

$$
r: \operatorname{NCW}^{-1}(X, Y) \rightarrow \operatorname{Map}_{L_{W} \mathrm{C}}(X, Y)
$$

sending such a hammock to a reduced hammock, in the sense of [13, Sec. 2.1]. The main theorem of this section is:

Theorem 2.13. Let $\mathrm{C}$ be a small incomplete category of fibrant objects with functorial path objects and functorial pullbacks of acyclic fibrations. Then for all objects $X, Y$ of $\mathrm{C}$, the maps

$$
N \mathrm{CW}_{\mathrm{f}}^{-1}(X, Y) \stackrel{N \iota}{\longrightarrow} N \mathrm{CW}^{-1}(X, Y) \stackrel{r}{\rightarrow} \operatorname{Map}_{L_{W} \mathrm{C}}(X, Y)
$$

are weak homotopy equivalences of simplicial sets.

Prop. 2.12 implies, of course, that the first map in (2.3) is a weak homotopy equivalence. To prove Thm. 2.13, we just need to show that the reduction map is a weak equivalence. We do this by showing that $\mathrm{C}$ admits a homotopy calculus of right fractions. Then our result will follow from a result of Dwyer and Kan [13, Prop. 6.2].

Let $i, j \geq 0$ be integers. Given objects $X, Y \in \mathrm{C}$ there is functor

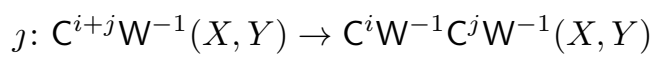

which sends a diagram of the form

$$
X \stackrel{f_{0}}{\sim} C_{1} \stackrel{f_{1}}{\longrightarrow} C_{2} \stackrel{f_{2}}{\longrightarrow} \cdots C_{j} \stackrel{f_{j}}{\longrightarrow} C_{j+1} \stackrel{f_{j+1}}{\longrightarrow} \cdots C_{i+j} \stackrel{f_{i+j}}{\longrightarrow} Y
$$

to the diagram

$$
X \stackrel{f_{0}}{\sim} C_{1} \stackrel{f_{1}}{\longrightarrow} C_{2} \stackrel{f_{2}}{\longrightarrow} \cdots C_{j} \stackrel{f_{j}}{\longrightarrow} C_{j+1} \stackrel{\mathrm{id}}{\sim} C_{j+1} \stackrel{f_{j+1}}{\longrightarrow} \cdots C_{i+j} \stackrel{f_{i+j}}{\longrightarrow} Y
$$


We abuse notation and denote by $\jmath: \mathrm{W}^{i+j} \mathrm{~W}^{-1}(X, Y) \rightarrow \mathrm{W}^{i} \mathrm{~W}^{-1} \mathrm{~W}^{j} \mathrm{~W}^{-1}(X, Y)$ the restriction of (2.4) to the subcategory $\mathrm{W}^{i+j} \mathrm{~W}^{-1}(X, Y)$. We recall [13, Sec. 6.1] that the pair $(C, W)$ admits a homotopy calculus of right fractions iff the induced maps on nerves

$$
\begin{gathered}
N C^{i+j} \mathrm{~W}^{-1}(X, Y) \stackrel{N_{J}}{\longrightarrow} N C^{i} \mathrm{~W}^{-1} \mathrm{C}^{j} \mathrm{~W}^{-1}(X, Y), \\
N \mathrm{~W}^{i+j} \mathrm{~W}^{-1}(X, Y) \stackrel{N_{J}}{\longrightarrow} N \mathrm{~W}^{i} \mathrm{~W}^{-1} \mathrm{C}^{j} \mathrm{~W}^{-1}(X, Y)
\end{gathered}
$$

are weak homotopy equivalences for all $i, j \geq 0$ and objects $X, Y \in \mathrm{C}$.

Proof of Thm. 2.13. We show (C, W) admits a homotopy calculus of right fractions by adopting the strategy used by Nikolaus, Schreiber, and Stevenson to prove their Thm. 3.61 in [29]. First, we observe that the proof of Prop. 2.12 can be easily generalized to show that the inclusions of subcategories

$$
\mathrm{C}^{i} \mathrm{~W}_{\mathrm{f}}^{-1} \stackrel{\iota}{\hookrightarrow} \mathrm{C}^{i} \mathrm{~W}^{-1}, \quad \mathrm{~W}^{i} \mathrm{~W}_{\mathrm{f}}^{-1} \stackrel{\iota}{\hookrightarrow} \mathrm{W}^{i} \mathrm{~W}^{-1}
$$

and

$$
\mathrm{C}^{i} \mathrm{~W}_{\mathrm{f}}^{-1} \mathrm{C}^{j} \mathrm{~W}_{\mathrm{f}}^{-1} \stackrel{\iota}{\hookrightarrow} \mathrm{C}^{i} \mathrm{~W}^{-1} \mathrm{C}^{j} \mathrm{~W}^{-1}, \quad \mathrm{~W}^{i} \mathrm{~W}_{\mathrm{f}}^{-1} \mathrm{~W}^{j} \mathrm{~W}_{\mathrm{f}}^{-1} \stackrel{\iota}{\hookrightarrow} \mathrm{W}^{i} \mathrm{~W}^{-1} \mathrm{~W}^{j} \mathrm{~W}^{-1}
$$

induce homotopy equivalences on the corresponding nerves. Next, we consider the restriction of the functor (2.4) $\mathrm{\jmath}: \mathrm{C}^{i+j} \mathrm{~W}^{-1}(X, Y) \rightarrow \mathrm{C}^{i} \mathrm{~W}^{-1} \mathrm{C}^{j} \mathrm{~W}^{-1}(X, Y)$ to the following subcategories:

$$
\mathrm{C}^{i+j} \mathrm{~W}_{\mathrm{f}}^{-1}(X, Y) \stackrel{\jmath}{\rightarrow} \mathrm{C}^{i} \mathrm{~W}_{\mathrm{f}}^{-1} \mathrm{C}^{j} \mathrm{~W}_{\mathrm{f}}^{-1}(X, Y),
$$

and

$$
\mathrm{W}^{i+j} \mathrm{~W}_{\mathrm{f}}^{-1}(X, Y) \stackrel{\jmath}{\rightarrow} \mathrm{W}^{i} \mathrm{~W}_{\mathrm{f}}^{-1} \mathrm{~W}^{j} \mathrm{~W}_{\mathrm{f}}^{-1}(X, Y) .
$$

Let $F: \mathrm{C}^{i} \mathrm{~W}_{\mathrm{f}}^{-1} \mathrm{C}^{j} \mathrm{~W}_{\mathrm{f}}^{-1}(X, Y) \rightarrow \mathrm{C}^{i+j} \mathrm{~W}_{\mathrm{f}}^{-1}(X, Y)$ be the functor that assigns to the diagram

$$
X \stackrel{f_{0}}{\sim} C_{1} \stackrel{f_{1}}{\longrightarrow} C_{2} \stackrel{f_{2}}{\longrightarrow} \cdots C_{j} \stackrel{f_{j}}{\longrightarrow} C_{j+1} \stackrel{f_{j+1}}{\stackrel{\sim}{\sim}} C_{j+2} \stackrel{f_{j+2}}{\longrightarrow} \cdots C_{i+j+1} \stackrel{f_{i+j+1}}{\longrightarrow} Y
$$

a diagram

$$
X \stackrel{g_{0}}{\stackrel{\sim}{\sim}} D_{1} \stackrel{g_{1}}{\longrightarrow} D_{2} \stackrel{g_{2}}{\longrightarrow} \cdots \longrightarrow D_{i+j} \stackrel{g_{i+j}}{\longrightarrow} Y
$$

obtained by taking the iterated pullback of the acyclic fibration $f_{j+1}$ :

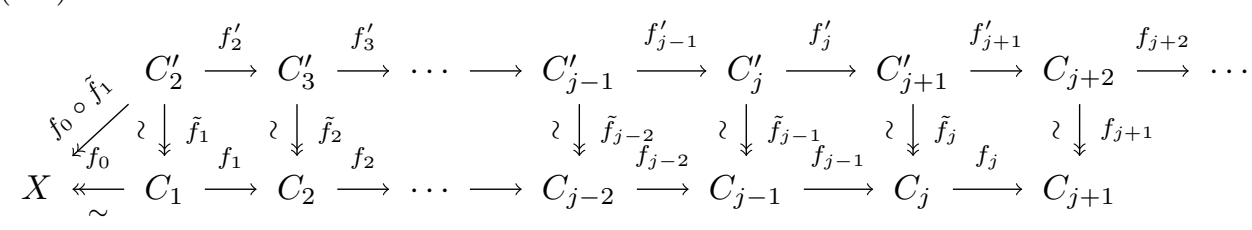

Hence, $D_{k}:=C_{k+1}^{\prime}$ if $k \leq j$, otherwise $D_{k}:=C_{k+1}$. And $g_{0}:=f_{0} \circ \tilde{f}_{1}$, $g_{k}:=f_{k+1}^{\prime}$ if $1 \leq k \leq j$, otherwise $g_{k}:=f_{k+1}$. Note $F$ is indeed a functor, since all the pullbacks in (2.9) are functorial. Furthermore, if all the $f_{k}$ are morphisms in $\mathrm{W}$, then so are the $g_{k}$ by the 2 out of 3 axiom. Hence $F$ restricts to a functor $\mathrm{W}^{i} \mathrm{~W}_{\mathrm{f}}^{-1} \mathrm{~W}^{j} \mathrm{~W}_{\mathrm{f}}^{-1}(X, Y) \rightarrow \mathrm{W}^{i+j} \mathrm{~W}_{\mathrm{f}}^{-1}(X, Y)$ 
There is a natural transformation $F \circ \jmath \rightarrow \mathrm{id}_{\mathrm{C}^{i+j} \mathrm{~W}_{\mathrm{f}}-1(X, Y)}$, where $\jmath$ is the functor (2.7), whose components are all identity morphisms. Indeed, if $f_{j+1}=\mathrm{id}_{C_{j+1}}$ in the diagram (2.9), then $\tilde{f}_{k}=\mathrm{id}_{C_{k-1}}$ for all $k \geq 1$. There is also a natural transformation $\jmath \circ F \rightarrow \mathrm{id}_{\mathrm{C}^{i} \mathrm{~W}_{\mathrm{f}}-1} \mathrm{C}^{j} \mathrm{~W}_{\mathrm{f}}-1(X, Y)$ whose components are the vertical maps in the following diagram:

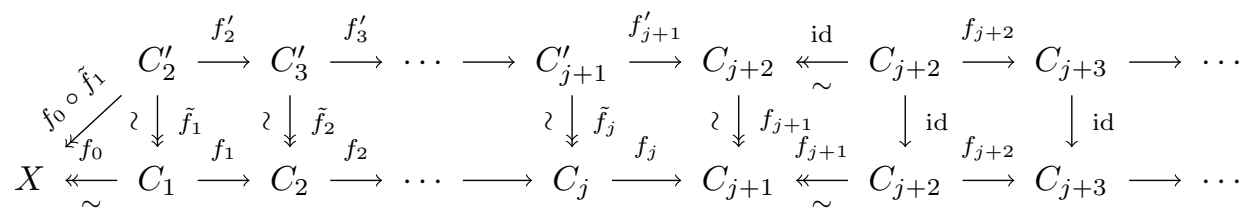

The existence of these natural transformations implies that the functors (2.7) and (2.8) induce homotopy equivalences on the corresponding nerves. Combining these with functors $(2.5),(2.6)$ and then taking the nerve, gives us commutative diagrams of simplicial sets.

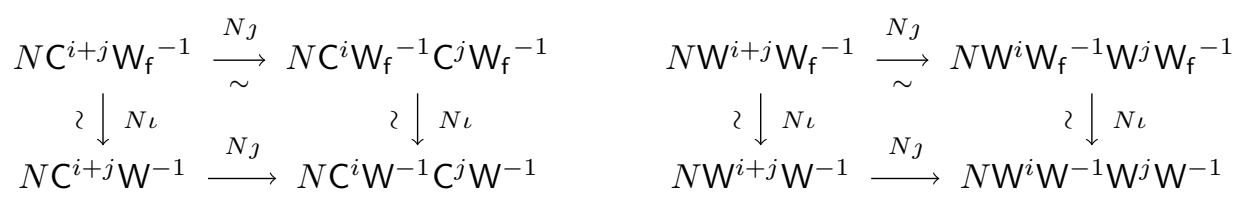

By 2 out of 3, the bottom horizontal morphisms in these diagrams are weak equivalences of simplicial sets. Therefore, $(\mathrm{C}, \mathrm{W})$ admits a homotopy calculus of right fractions.

\section{Higher groupoids AND KAN FIBRATIONS}

In this section, we recall Henriques' definition [17] of a higher groupoid object in a (large) category $M$ equipped with a pretopology $\mathcal{T}$.

3.1. Preliminaries and notation. If $M$ is a small category, we denote by $P S h(M)$ the category of presheaves on $\mathrm{M}$. The functor $\mathbf{y}: \mathrm{M} \rightarrow \mathrm{PSh}(\mathrm{M}), X \mapsto \mathbf{y} X=$ $\operatorname{hom}_{\mathrm{M}}(-, X)$ denotes the Yoneda embedding, which identifies objects in $M$ with the representable presheaves.

We denote by $s \mathrm{M}$ the category of simplicial objects in $\mathrm{M}$, i.e. the category of contravariant functors $\Delta \rightarrow \mathrm{M}$, where $\Delta$ is the category of finite ordinals

$$
[0]=\{0\}, \quad[1]=\{0,1\}, \quad \ldots, \quad[n]=\{0,1, \ldots, n\}, \quad \ldots,
$$

with order-preserving maps. In particular, $s$ Set is the category of simplicial sets. For $m \geq 0$, the simplicial sets $\Delta^{m}$ and $\partial \Delta^{m}$ are the simplicial $m$-simplex and its boundary, respectively:

$$
\begin{aligned}
\left(\Delta^{m}\right)_{n} & =\{f:(0,1, \ldots, n) \rightarrow(0,1, \ldots, m) \mid f(i) \leq f(j) \text { for all } i \leq j\}, \\
\left(\partial \Delta^{m}\right)_{n} & =\left\{f \in\left(\Delta^{m}\right)_{n} \mid\{0, \ldots, m\} \nsubseteq\{f(0), \ldots, f(n)\}\right\} .
\end{aligned}
$$

For $m>0$ and $0 \leq j \leq m$, the horn $\Lambda_{j}^{m}$ is the simplicial set obtained from the $m$-simplex $\Delta^{m}$ by taking away its interior and its $j$ th face:

$$
\left(\Lambda_{j}^{m}\right)_{n}=\left\{f \in\left(\Delta^{m}\right)_{n} \mid\{0, \ldots, j-1, j+1, \ldots, m\} \nsubseteq\{f(0), \ldots, f(n)\}\right\} .
$$


3.2. Pretopologies. An $n$-groupoid in $\mathrm{M}$ is special kind of simplicial object in $\mathrm{M}$. The precise definition requires us to equip $\mathrm{M}$ with extra structure, which also allows us to define sheaves on $\mathrm{M}$.

Definition 3.1. Let $M$ be a category with coproducts and a terminal object $*$. A pretopology on $\mathrm{M}$ is a collection $\mathcal{T}$ of arrows, called covers, with the following properties:

(1) isomorphisms are covers;

(2) the composite of two covers is a cover;

(3) pullbacks of covers are covers; more precisely, for a cover $U \rightarrow X$ and an arrow $Y \rightarrow X$, the pull-back $Y \times_{X} U$ exists in $\mathrm{M}$ and the canonical map $Y \times{ }_{X} U \rightarrow Y$ is a cover;

(4) for any object $X \in \mathrm{M}$, the map $X \rightarrow *$ is a cover.

What we call a pretopology is called a "singleton Grothendieck pretopology" in [43], and was first defined in [17, Def. 2.1]. Every pretopology in our sense gives a Grothendieck pretopology in the classical sense.

Let $(\mathrm{M}, \mathcal{T})$ be a category equipped with a pretopology. A presheaf $F \in \operatorname{PSh}(\mathrm{M})$ is a sheaf if and only if for every cover $U \rightarrow X, F(X)$ is the equalizer of the diagram

$$
F(U) \rightrightarrows F\left(U \times_{X} U\right) .
$$

We denote by $\operatorname{Sh}(\mathrm{M}) \subseteq \mathrm{PSh}(\mathrm{M})$ the full subcategory of sheaves on $(\mathrm{M}, \mathcal{T})$. A pretopology is subcanonical iff every representable presheaf is a sheaf. In this paper, all pretopologies are assumed to be subcanonical.

Also, we will never assume $\mathrm{M}$ has limits. Therefore, we take limits of diagrams in $\mathrm{M}$ by first showing that the limit of the corresponding diagram in $\mathrm{Sh}(\mathrm{M})$ of representable presheaves is representable, and then using the fact that the functor y preserves limits.

3.3. Pretopologies for Banach manifolds. We denote by Mfd the category whose objects are Banach manifolds, in the sense of [25, Ch 2.1], and whose morphisms are smooth maps. (We could also consider $C^{r}$ maps as well.) A morphism $f: X \rightarrow Y$ between manifolds is a submersion iff for all $x \in X$, there exists an open neighborhood $U_{x}$ of $x$, an open neighborhood $V_{f(x)}$ of $f(x)$, and a local section $\sigma: V_{f(x)} \rightarrow U_{x}$ of $f$ at $x$. That is, $\sigma$ is a morphism in Mfd such that $f \circ \sigma=$ id and $\sigma(f(x))=x$. Note that we may always take $U_{x}$ to be the connected component of of $f^{-1}\left(V_{f(x)}\right)$ containing $x$.

It is a result of Henriques ([17, Cor. 4.4]), that the collection $\mathcal{T}_{\text {subm }}$ of surjective submersions is a subcanonical pretopology for the category Mfd.

Remark 3.2. Another example of a subcanonical pretopology on Mfd is the pretopology of open covers $\mathcal{T}_{\text {open }}$. Since every cover in the surjective submersion pretopology can be refined by a cover in the pretopology of open covers (see Example B.6), every sheaf on $\left(\mathrm{Mfd}, \mathcal{T}_{\text {open }}\right)$ is also a sheaf on $\left(\mathrm{Mfd}, \mathcal{T}_{s s}\right)$ and vice versa. See also [30, Prop. 2.17].

Examples of subcanonical pretopologies for other categories of geometric interest can be found in Table 1 of [43].

3.3.1. Technicalities involving large categories. Strictly speaking, the categories $\mathrm{Sh}(\mathrm{M})$ and $\mathrm{PSh}(\mathrm{M})$ are not well-defined if $\mathrm{M}$ is not small. In our main example of interest, $M$ will be the large category of Banach manifolds, so we will need a good 
theory of sheaves over a large category. The set-theoretic technicalities involved with sheaves over large categories can be subtle. For example, the sheafification functor may not be well-defined for presheaves over a large site since a priori it requires taking colimits over proper classes. The standard workaround is to use Grothendieck universes, and in particular, to appeal to the Universe Axiom, that allows one to take colimits in an ambient larger universe in which classes are sets. However, this larger universe is by no means canonical, and the resulting colimit may very well depend on the choice of larger universe. See [40] for such an example involving sheaves for the fpqc topology in algebraic geometry.

We show in Appendix B that all results in this paper are independent of choice of universe, provided our pretopology $\mathcal{T}$ on the large category $M$ admits what we call a "small refinement" (Def. B.5). Indeed, an example of such a pretopology is the surjective submersion pretopology on the category of Banach manifolds. (See Example B.6.)

From here on, we always assume that the pretopology being considered admits a small refinement. This allows the reader to ignore all set-theoretic issues, and treat $(\mathrm{M}, \mathcal{T})$ as if it was a pretopology on a small category.

3.4. Kan fibrations and higher groupoids in $(\mathrm{M}, \mathcal{T})$. Here we recall Henriques' definition of Kan fibration and $n$-groupoid, which is based on the work of Duskin [12] and Glenn [16]. Let $(\mathrm{M}, \mathcal{T})$ be a category equipped with a pretopology. In what follows, if $K$ is a simplicial set and $X$ is a simplicial object in $\mathrm{M}$, then we denote by $\operatorname{Hom}(K, X)$ the sheaf

$$
\operatorname{Hom}(K, X)(U):=\operatorname{hom}_{s \mathrm{M}}(K \otimes U, X)
$$

where $K \otimes U$ is the simplicial object $(K \otimes U)_{n}:=\coprod_{K_{n}} U$. (See Prop. 4.5 for other characterizations of this sheaf.) Note that $\operatorname{Hom}(K, X)$ may not be representable.

More generally, suppose $i: A \rightarrow B$ is a map between simplicial sets and $f: X \rightarrow$ $Y$ is a morphism of simplicial objects in M. Denote by

$$
\operatorname{Hom}(A \stackrel{i}{\rightarrow} B, X \stackrel{f}{\rightarrow} Y):=\operatorname{Hom}(A, X) \times \operatorname{Hom}(A, Y) \operatorname{Hom}(B, Y)
$$

the sheaf which assigns to an object $U \in \mathrm{M}$ the set of commuting squares in $s \mathrm{M}$ of the form

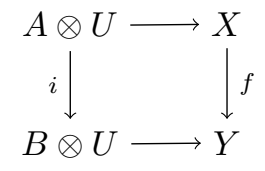

There is a canonical map

$$
\operatorname{Hom}(B, X) \stackrel{\left(i^{*}, f_{*}\right)}{\longrightarrow} \operatorname{Hom}(A \rightarrow B, X \rightarrow Y)
$$

induced by pre and post composition with $i: A \rightarrow B$ and $f: X \rightarrow Y$, respectively.

Definition 3.3 (Def. 2.3 [17]). A morphism $f: X \rightarrow Y$ of simplicial objects in a category equipped with a pretopology $(\mathbf{M}, \mathcal{T})$ satisfies the $\operatorname{Kan}$ condition $\operatorname{Kan}(m, j)$ iff the sheaf $\operatorname{Hom}\left(\Lambda_{j}^{m} \rightarrow \Delta^{m}, X \rightarrow Y\right)$ is representable and the canonical map (i.e., the horn projection)

$$
X_{m}=\operatorname{Hom}\left(\Delta^{m}, X\right) \stackrel{\left(\iota_{m, j}^{*}, f_{*}\right)}{\longrightarrow} \operatorname{Hom}\left(\Lambda_{j}^{m} \stackrel{\iota_{m, j}}{\longrightarrow} \Delta^{m}, X \stackrel{f}{\rightarrow} Y\right)
$$


is a cover. The morphism $f: X \rightarrow Y$ satisfies the unique Kan condition $\operatorname{Kan} !(m, j)$ iff the canonical map in (3.3) is an isomorphism. We say $f: X \rightarrow Y$ is a Kan fibration iff it satisfies $\operatorname{Kan}(m, j)$ for all $m \geq 1,0 \leq j \leq m$.

Definition 3.4 (Def. 2.3 [17]). A simplicial object $X \in s \mathrm{M}$ is a higher groupoid in $(\mathrm{M}, \mathcal{T})$, or more precisely, an $n$-groupoid object in $(\mathrm{M}, \mathcal{T})$ for $n \in \mathbb{N} \cup\{\infty\}$ iff the unique morphism

$$
X \rightarrow *
$$

satisfies the $\operatorname{Kan}$ condition $\operatorname{Kan}(m, j)$ for $1 \leq m \leq n, 0 \leq j \leq m$, and the unique Kan condition Kan! $(m, j)$ for all $m>n, 0 \leq j \leq m$. An $n$-group object in $(\mathrm{M}, \mathcal{T})$ is an $n$-groupoid object $X$, such that $X_{0}=*$, where $*$ is the terminal object in M.

In other words, $X$ is an $n$-groupoid if the sheaf $\operatorname{Hom}\left(\Lambda_{j}^{m}, X\right)$ is representable and the restriction map

$$
\operatorname{Hom}\left(\Delta^{m}, X\right) \rightarrow \operatorname{Hom}\left(\Lambda_{j}^{m}, X\right)
$$

is a cover for all $1 \leq m \leq n, 0 \leq j \leq m$ and an isomorphism for all $m>n$, $0 \leq j \leq m$.

3.5. Representability results. Now we record some useful tools for proving representability. Similar results can be found in the work of Behrend and Getzler [3], Wolfson [42], and Zhu [43]. What follows is reminiscent of the use of anodyne extensions in simplicial sets.

Definition 3.5. The inclusion $\iota: S \hookrightarrow T$ of a simplicial subset $S$ into a finitely generated simplicial set $T$ is a collapsible extension iff it is the composition of inclusions of simplicial subsets

$$
S=S_{0} \hookrightarrow S_{1} \hookrightarrow \cdots \hookrightarrow S_{l}=T
$$

where each $S_{i}$ is obtained from $S_{i-1}$ by filling a horn. That is, for each $i=1, \ldots, l$, there is a horn $\Lambda_{j}^{m}$ and a map $\Lambda_{j}^{m} \rightarrow S_{i-1}$ such that $S_{i}=S_{i-1} \sqcup_{\Lambda_{j}^{m}} \Delta^{m}$. If the inclusion of a point into a finitely-generated simplicial set $T$ is a collapsible extension, then we say $T$ is collapsible. Similarly, we say $\iota: S \hookrightarrow T$ is a boundary extension iff it is the composition of inclusions of simplicial subsets

$$
S=S_{0} \hookrightarrow S_{1} \hookrightarrow \cdots \hookrightarrow S_{l}=T
$$

where each $S_{i}$ is obtained from $S_{i-1}$ by filling a boundary. That is, for each $i=$ $1, \ldots, l$, there is a $m \geq 0$ and a map $\partial \Delta^{m} \rightarrow S_{i-1}$ such that $S_{i}=S_{i-1} \sqcup_{\partial \Delta^{m}} \Delta^{m}$.

Collapsible extensions are called "expansions" in [42]. We follow the terminology that appears in [26, Sec. 2.6]. (A more detailed study of these morphisms can be found there.) Other examples of collapsible extensions are the " $m$-expansions" in [3, Def. 3.7].

Clearly a collapsible extension is a boundary extension since the natural inclusion $\Lambda_{j}^{n} \rightarrow \Delta^{n}$ can be decomposed into two boundary extensions $\Lambda_{j}^{n} \rightarrow \partial \Delta^{n} \rightarrow \Delta^{n}$. Also, if $S \hookrightarrow T$ and $T \hookrightarrow U$ are both collapsible extensions (or boundary extensions), then so is their composition $S \hookrightarrow U$.

We have the following fact:

Lemma 3.6 (Lemma 2.44, [26]). The inclusion of any face $\Delta^{k} \rightarrow \Delta^{n}$ is a collapsible extension for $0 \leq k<n$. 
We will use the following two lemmas to solve most of the representability issues in this paper. They are similar to Lemma 2.4 in [17].

Lemma 3.7. Let $S \hookrightarrow T$ be a collapsible extension and let $X$ be a higher groupoid in $(\mathrm{M}, \mathcal{T})$. If $\operatorname{Hom}(S, X)$ is representable, then $\operatorname{Hom}(T, X)$ is representable as well and the restriction map $\operatorname{Hom}(T, X) \rightarrow \operatorname{Hom}(S, X)$ is a cover.

Proof. Let $S=S_{0} \subset S_{1} \subset \cdots \subset S_{l}=T$ be a filtration as in Definition 3.5. Since composites of covers are again covers, we may assume without loss of generality that $l=1$, i.e., $T=S \cup_{\Lambda_{j}^{n}} \Delta^{n}$ for some $n, j$. Note that the functor $\operatorname{Hom}(-, X)$ (3.1) sends colimits of simplicial sets to limits of sheaves. Hence, we have

$$
\operatorname{Hom}(T, X)=\operatorname{Hom}(S, X) \times_{\operatorname{Hom}\left(\Lambda_{j}^{n}, X\right)} \operatorname{Hom}\left(\Delta^{n}, X\right) .
$$

Since $X$ is a higher groupoid, $\operatorname{Hom}\left(\Delta^{n}, X\right) \rightarrow \operatorname{Hom}\left(\Lambda_{j}^{n}, X\right)$ is a cover between representable sheaves, and hence, the axioms of a pretopology imply that $\operatorname{Hom}(T, X)$ is representable and that $\operatorname{Hom}(T, X) \rightarrow \operatorname{Hom}(S, X)$ is a cover.

Remark 3.8. In particular, if $X$ is a $k$-groupoid for $k<\infty$, and $T=S \cup_{\Lambda_{j}^{n}} \Delta^{n}$ with $n>k$, and $\operatorname{Hom}(S, X)$ is representable, then the proof of Lemma 3.7 implies that $\operatorname{Hom}(T, X) \rightarrow \operatorname{Hom}(S, X)$ is not just a cover, but an isomorphism.

The next lemma concerns the representability of the sheaf (3.2).

Lemma 3.9. Let $S$ be a collapsible simplicial subset of $\Delta^{k}, X$ a simplicial object in $\mathrm{M}$, and $Y$ a higher groupoid in $\mathrm{M}$. If $f: X \rightarrow Y$ is a morphism which satisfies $\operatorname{Kan}(m, j)$ for all $m<k$ and $0 \leq j \leq m$, then the sheaf $\operatorname{Hom}\left(S \hookrightarrow \Delta^{k}, X \stackrel{f}{\rightarrow} Y\right)$ is representable.

Proof. First note that the statement is identical to that of [17, Lemma 2.4] except that we do not require $X_{0}=Y_{0}=*$. So, we can proceed exactly as in the proof of [17, Lemma 2.4], but with a different verification of the base case for the induction. For this, we consider $S_{0}=* \hookrightarrow \Delta^{k}$ and observe that the sheaf Hom $\left(* \hookrightarrow \Delta^{k}, X \stackrel{f}{\rightarrow}\right.$ $Y$ ) is represented by the pullback $X_{0} \times_{Y_{0}} Y_{k}$ in $\mathrm{M}$, which exists in $\mathrm{M}$ since Lemmas 3.6 and 3.7. imply that $Y_{k} \rightarrow Y_{0}$ is a cover.

Remark 3.10. The horn $\Lambda_{j}^{n} \subset \Delta^{n}$ is collapsible. Hence, if $Y$ is a higher groupoid and $f: X \rightarrow Y$ is a simplicial morphism satisfying the $\operatorname{Kan}$ condition $\operatorname{Kan}(m, j)$ for $1 \leq m<n$, then Lemma 3.9 implies that $\operatorname{Hom}\left(\Lambda_{j}^{n} \rightarrow \Delta^{n}, X \rightarrow Y\right)$ is automatically representable. Similarly, if $X$ is a simplicial object and if $X \rightarrow *$ satisfies the Kan condition $\operatorname{Kan}(m, j)$ for $1 \leq m<n$, then Lemma 3.9 implies that $\operatorname{Hom}\left(\Lambda_{j}^{n}, X\right)$ is automatically representable.

\section{Points for Categories with Pretopologies}

We begin this section by considering certain functors called "points" for a category equipped with a pretopology. This will allow us to make comparisons between the homotopy theory of higher groupoid objects and that of simplicial sets. A point can be thought of as a generalization of the functor which sends a sheaf on a space to its stalk at a particular point. The notion originates in topos theory. See, for example, [22, C.2.2, p. 555] and [28, VII.5].

We will not need all of the topos theory formalism here, so our presentation is quite abbreviated and self-contained. Our motivation stems from the use of points 
in the homotopy theory of simplicial sheaves over the category of finite-dimensional manifolds (e.g. [11, 29]).

Definition 4.1. Let $(\mathrm{M}, \mathcal{T})$ be a category equipped with a pretopology.

(1) A point of $(M, \mathcal{T})$ is a functor

$$
\mathrm{p}: \mathrm{Sh}(\mathrm{M}) \rightarrow \text { Set }
$$

which preserves finite limits and small colimits.

(2) A collection of points $\mathcal{P}$ of $(\mathrm{M}, \mathcal{T})$ is jointly conservative iff a morphism $\phi: F \rightarrow G$ in $\operatorname{Sh}(\mathrm{M})$ is an isomorphism if and only if for all $\mathrm{p} \in \mathcal{P}$

$$
\mathrm{p}(\phi): \mathrm{p}(F) \rightarrow \mathrm{p}(G)
$$

is an isomorphism of sets.

If $X$ is a simplicial object in $(M, \mathcal{T})$ and $\mathrm{p}: \mathrm{Sh}(\mathrm{M}) \rightarrow$ Set is a point, we denote by $\mathrm{p} X$ the simplicial set

$$
\mathrm{p} X_{n}:=\mathrm{p}\left(\mathbf{y} X_{n}\right)
$$

4.1. Points for Banach manifolds. Our main example, the category of Banach manifolds equipped with the pretopology of surjective submersions, admits a jointly conservative collection of points. If we were only considering finite-dimensional manifolds, then these points would be the same as those used in [11, Def. 3.4.6].

Let $V \in$ Ban be a Banach space, and denote by $B_{V}(r)$ the open ball of radius $r$ about the origin in $V$. Let $\left(\mathrm{Mfd}, \mathcal{T}_{\text {subm }}\right)$ denote the category of Banach manifolds equipped with the surjective submersion pretopology, and denote by $\mathrm{p}_{V}: \mathrm{Sh}(\mathrm{Mfd}) \rightarrow$ Set the functor

$$
\mathrm{p}_{V}(F)=\operatorname{colim}_{r \rightarrow 0} F\left(B_{V}(r)\right) .
$$

\section{Proposition 4.2.}

(1) For every Banach space $V$, the functor $\mathrm{p}_{V}: \mathrm{Sh}(\mathrm{Mfd}) \rightarrow$ Set preserves finite limits and small colimits.

(2) The collection of points $\mathcal{P}_{\mathrm{Ban}}:=\left\{\mathrm{p}_{V} \mid V \in \operatorname{Ban}\right\}$ is jointly conservative.

Proof. Since $\mathrm{p}_{V}$ is a filtered colimit, it commutes with finite limits and small colimits. Now let $\phi: F \rightarrow G$ be a morphism of sheaves such that for all $V \in$ Ban

$$
\mathrm{p}_{V}(\phi): \mathrm{p}_{V} F \rightarrow \mathrm{p}_{V} G
$$

is bijection. In particular, injectivity implies that if $x \in F\left(B_{V}\left(r_{x}\right)\right)$ and $y \in$ $F\left(B_{V}\left(r_{y}\right)\right)$ such that $\mathrm{p}_{V}(\phi)(\bar{x})=\mathrm{p}_{V}(\phi)(\bar{y})$, then there exists $r \leq r_{x}$ and $r \leq r_{y}$ such that $i_{x}^{*} x=i_{y}^{*} y$, where $i_{x}: B_{V}(r) \rightarrow B_{V}\left(r_{x}\right)$ and $i_{y}: B_{V}(r) \rightarrow B_{V}\left(r_{y}\right)$ are the inclusions.

We show $\phi: F \rightarrow G$ is injective. Let $M \in \operatorname{Mfd}$ and $x, y \in F(M)$ such that $\phi_{M}(x)=\phi_{M}(y)$. By the usual arguments, for each $m \in M$ there exists an open neighborhood $U_{m}$ of $m$, a Banach space $V_{m}$, a radius $r_{m}>0$ and a diffeomorphism $\psi_{m}: B_{V_{m}}\left(r_{m}\right) \stackrel{\cong}{\rightrightarrows} U_{m}$ such that $\psi_{m}(0)=m$. Hence, we have a cover

$$
\coprod_{m \in M} B_{V_{m}}\left(r_{m}\right) \stackrel{\left(i_{m}\right)}{\longrightarrow} M
$$

where $i_{m}: B_{V_{m}}\left(r_{m}\right) \rightarrow M$ is the composition of $\psi_{m}$ with the inclusion. So for each $m \in M$

$$
\phi_{B_{V_{m}}\left(r_{m}\right)}\left(i_{m}^{*} x\right)=\phi_{B_{V_{m}}\left(r_{m}\right)}\left(i_{m}^{*} y\right)
$$


which implies

$$
p_{V_{m}}^{*}\left(\phi_{B_{V_{m}}\left(r_{m}\right)}\right)\left(\overline{i_{m}^{*} x}\right)=p_{V_{m}}^{*}\left(\phi_{B_{V_{m}}\left(r_{m}\right)}\right)\left(\overline{i_{m}^{*} y}\right) .
$$

Therefore, there exists a smaller ball $B_{V_{m}}\left(r_{m}^{\prime}\right) \subseteq B_{V_{m}}\left(r_{m}\right)$ such that the restrictions of $x$ and $y$ to $B_{V_{m}}\left(r_{m}^{\prime}\right)$ are equal. Since

$$
\coprod_{m \in M} B_{V_{m}}\left(r_{m}\right) \stackrel{\left(i_{m}\right)}{\longrightarrow} M
$$

is a cover, and $F$ is a sheaf, we conclude $x=y$.

Now we show $\phi: F \rightarrow G$ is surjective. Let $M \in \mathrm{Mfd}$ and $y \in G(M)$. We use the cover (4.2) as before, so that $i_{m}^{*} y \in G\left(B_{V_{m}}\left(r_{m}\right)\right)$. Since $\mathrm{p}_{V_{m}} \phi: \mathrm{p}_{V_{m}} F \rightarrow \mathrm{p}_{V_{m}} G$ is onto, there exists $r_{m}^{\prime} \leq r_{m}$ and $x_{m} \in F\left(B_{V_{m}}\left(r_{m}\right)\right)$ such that $\phi\left(x_{m}\right)=j_{m}^{*} y$ where $j_{m}: B_{V_{m}}\left(r_{m}^{\prime}\right) \rightarrow M$ is the composition of $i_{m}$ with the inclusion $B_{V_{m}}\left(r_{m}^{\prime}\right) \hookrightarrow$ $B_{V_{m}}\left(r_{m}\right)$.

Consider the pullback $W \times_{M} W \underset{p_{2}}{\stackrel{p_{1}}{\rightrightarrows}} W$, where $W$ is the cover $\coprod_{m \in M} B_{V_{m}}\left(r_{m}^{\prime}\right)$. We claim $p_{1}^{*}\left(\left\{x_{m}\right\}\right)=p_{2}^{*}\left(\left\{x_{m}\right\}\right)$. Indeed, since $y$ is a global section over $M$, we have the equalities

$$
\phi\left(p_{1}^{*}\left(\left\{x_{m}\right\}\right)\right)=p_{1}^{*} \phi\left(\left(\left\{x_{m}\right\}\right)\right)=p_{1}^{*}\left(\left\{j_{m}^{*} y\right\}\right)=p_{2}^{*}\left(\left\{j_{m}^{*} y\right\}\right)=\phi\left(p_{2}^{*}\left(\left\{x_{m}\right\}\right)\right) .
$$

Therefore, since $\phi$ is one to one, we conclude $p_{1}^{*}\left(\left\{x_{m}\right\}\right)=p_{2}^{*}\left(\left\{x_{m}\right\}\right)$. And since $F$ is a sheaf, there exists a section $x \in F(M)$ such that $j_{m}^{*} \phi(x)=j_{m}^{*} y$, and hence $\phi(x)=y$.

Remark 4.3. It follows from Remark 3.2 that Prop. 4.2 also implies that the collection of functors (4.1) is jointly conservative for ( $\left.\mathrm{Mfd}, \mathcal{T}_{\text {open }}\right)$.

4.2. Matching objects and stalkwise Kan fibrations. We next recall how points of $(\mathrm{M}, \mathcal{T})$ take "matching objects" for simplicial sheaves to matching objects for simplicial sets. We also describe the relationship between the sheaf $\operatorname{Hom}(K, X)$, defined in (3.1), for a simplicial object $X \in s \mathrm{M}$ and the corresponding matching object for the representable simplicial sheaf $\mathbf{y} X$.

Definition 4.4. Given a simplicial set $K \in s$ Set and a simplicial sheaf $F \in s \operatorname{Sh}(\mathrm{M})$, their matching object $M_{K}(F)$ is the sheaf

$$
M_{K}(F)(U):=\operatorname{hom}_{s \operatorname{Set}}(K, F(U)) \text {. }
$$

If $F \in s \operatorname{Sh}(\mathrm{M})$ is a simplicial sheaf and $K \in s$ Set is a simplicial set, then we denote by $F_{m}^{K_{n}}$ the sheaf

$$
U \mapsto F_{m}^{K_{n}}(U):=\prod_{K_{n}} F_{m}(U)
$$

Hence, for each $m, n$, and element $x \in K_{n}$ we have the canonical projection $\pi_{m}^{n}(x): F_{m}^{K_{n}} \rightarrow F_{m}$. For any such $F$ and $K$, there are two maps of sheaves:

$$
\alpha_{F}, \alpha_{K}: \prod_{m \geq 0} F_{m}^{K_{m}} \rightarrow \prod_{\theta_{m n}} F_{m}^{K_{n}},
$$

where the latter product is taken over all morphisms $\theta_{m n}:[m] \rightarrow[n]$ in the category $\Delta$. The maps $\alpha_{F}$ and $\alpha_{K}$ are defined in the following way: If $U \in \mathrm{M}$, then $F_{m}^{K_{n}}(U)=\operatorname{hom}_{\text {Set }}\left(K_{n}, F_{m}(U)\right)$. So let $\bar{f}=\left(f_{m}\right) \in \prod_{m} F_{m}^{K_{m}}(U)$. Then, the projections of $\alpha_{F}(\bar{f})$ and $\alpha_{K}(\bar{f})$ to the factor $F_{m}^{K_{n}}(U)$ labeled by a morphism $\theta_{m n}:[m] \rightarrow[n]$ are $F\left(\theta_{m n}\right)\left(f_{n}\right)$ and $f_{m} \circ K\left(\theta_{m n}\right)$, respectively. 
We now record some basic facts about the matching object $M_{K}(F)$.

\section{Proposition 4.5.}

(1) For any simplicial sheaf $F$ and morphism of simplicial sets $\gamma: K \rightarrow L$, there is a natural morphism of sheaves $M_{\gamma}: M_{L}(F) \rightarrow M_{K}(F)$.

(2) For any simplicial sheaf $F$ and simplicial set $K, M_{K}(F)$ is the equalizer of the diagram

$$
\prod_{m \geq 0} F_{m}^{K_{m}} \underset{\alpha_{K}}{\stackrel{\alpha_{F}}{\rightleftarrows}} \prod_{\theta:[m] \rightarrow[n]} F_{m}^{K_{n}}
$$

Moreover, if $K$ is a finitely generated simplicial set of dimension $M$, then $M_{K}(F)$ is the equalizer of the diagram

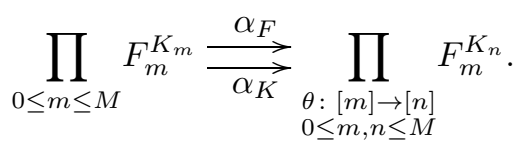

(3) If $X$ is a simplicial object in $\mathrm{M}$, and $y X$ is the representable simplicial sheaf $y X(U)_{n}=\operatorname{hom}_{\mathrm{M}}\left(U, X_{n}\right)$, then there is a unique natural isomorphism of sheaves $M_{K}(y X) \cong \operatorname{Hom}(K, X)$, where $\operatorname{Hom}(K, X)$ is the sheaf

$$
\operatorname{Hom}(K, X)(U):=\operatorname{hom}_{s \mathrm{M}}(K \otimes U, X)
$$

previously introduced in (3.1), and $K \otimes U$ is the simplicial object $(K \otimes U)_{n}:=$ $\amalg_{K_{n}} U$.

Proof. Statement (1) is obvious, as is the proof that $M_{K}(F)$ is the equalizer of (4.3). If $K$ is finitely generated, then one shows $M_{K}(F)$ is the equalizer of (4.4) by using the fact that every simplex in $K$ can be uniquely written as a non-degenerate simplex composed with a degeneracy map (the "Eilenberg-Zilber Lemma"). Finally, (3) follows by showing that $\operatorname{Hom}(K, X)$ is the equalizer of (4.3) when $F=\mathbf{y} X$.

Corollary 4.6. Let $X$ be a higher groupoid object in $(\mathrm{M}, \mathcal{T})$ and $K$ a finitely generated simplicial set. Then for each point $\mathrm{p}: \mathrm{Sh}(\mathrm{M}) \rightarrow$ Set there is an unique natural isomorphism of sets

$$
\mathrm{p} \operatorname{Hom}(K, X) \cong \operatorname{hom}_{s \text { Set }}(K, \mathrm{p} X)
$$

Proof. For any simplicial set $K$, Prop. 4.5 implies that

$$
\mathrm{p} \operatorname{Hom}(K, X) \cong \mathrm{p} M_{K}(\mathbf{y} X) \text {. }
$$

If $K$ is finitely generated, then for any $n, m \geq 0, \mathbf{y} X_{m}^{K_{n}}$ is a finite product of sheaves, and so Prop. 4.5 implies that $M_{K}(\mathbf{y} X)$ is an equalizer of finite limits of sheaves. By definition, the functor $\mathrm{p}$ preserves finite limits. Hence,

$$
\mathrm{p} M_{K}(\mathbf{y} X) \cong \mathrm{eq}\left(\prod_{0 \leq m \leq M}\left(\mathrm{p} X_{m}\right)^{K_{m}} \frac{\mathrm{p} \alpha_{y} X}{\overrightarrow{\mathrm{p} \alpha_{K}}} \prod_{\substack{\theta:[m] \rightarrow[n] \\ 0 \leq m, n \leq M}}\left(\mathrm{p} X_{m}\right)^{K_{n}}\right) .
$$

A direct computation shows that the equalizer on the right-hand side of (4.6) is simply $\operatorname{hom}_{s \text { Set }}(K, \mathrm{p} X)$. 
A jointly conservative collection of points allows us to compare Kan fibrations of higher groupoids in $(\mathrm{M}, \mathcal{T})$ to the usual Kan fibrations of simplicial sets. We first recall a few different notions of "surjection" for sheaves.

Definition 4.7. Let $(\mathrm{M}, \mathcal{T})$ be a category equipped with a pretopology.

(1) A morphism of sheaves $\phi: F \rightarrow G$ is a local surjection iff for every object $C \in \mathrm{M}$ and every element $y \in G(C)$, there exists a cover $U \stackrel{f}{\rightarrow} C$ such that the element $f^{*} y \in G(U)$ is in the image of $\phi_{U}: F(U) \rightarrow G(U)$.

(2) If $\mathcal{P}$ is a collection of jointly conservative points for $(\mathrm{M}, \mathcal{T})$, then a morphism of sheaves $\phi: F \rightarrow G$ is a stalkwise surjection with respect to $\mathcal{P}$ iff for all $\mathrm{p} \in \mathcal{P}$ the function

$$
\mathrm{p}(\phi): \mathrm{p} F \rightarrow \mathrm{p} G
$$

is a surjection.

Lemma 4.8. Let $(\mathrm{M}, \mathcal{T})$ be a category equipped with a pretopology.

(1) If $\phi: F \rightarrow G$ is a local surjection of sheaves, then $\phi$ is an epimorphism of sheaves.

(2) If $\phi: F \rightarrow G$ is an epimorphism of sheaves, then $\phi$ is a stalkwise surjection with respect to any collection of jointly conservative points $\mathcal{P}$ of $(\mathrm{M}, \mathcal{T})$.

(3) Let $f: U \rightarrow C$ be a cover in $(\mathrm{M}, \mathcal{T})$. The induced morphism of sheaves $f_{*}: \mathbf{y}(U) \rightarrow \mathbf{y}(C)$ is a stalkwise surjection with respect to any collection of jointly conservative points.

Proof. (1) Suppose $\alpha, \beta: G \rightarrow H$ are morphisms of sheaves such that $\alpha \circ \phi=\beta \circ \phi$. We wish to show $\alpha=\beta$. Let $C \in \mathrm{M}$ and $y \in G(C)$. Let $U \stackrel{f}{\rightarrow} C$ be a cover such that there exists $x \in F(U)$ such that $\phi_{U}(x)=f^{*}(y) \in G(U)$. Since $\alpha_{U} \circ \phi_{U}=\beta_{U} \circ \phi_{U}$, we have

$$
\alpha_{U}\left(f^{*} y\right)=\beta_{U}\left(f^{*} y\right) \in H(U) .
$$

Hence, $f^{*} \alpha_{C}(y)=f^{*} \beta_{C}(y)$. Since $H$ is a sheaf, and $U$ is a cover, we conclude $\alpha_{C}(y)=\beta_{C}(y)$.

(2) A morphism $\phi: F \rightarrow G$ is an epimorphism if and only if the diagram

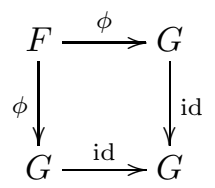

is a pushout. By definition, a point $\mathrm{p}: \mathrm{Sh}(\mathrm{M}) \rightarrow$ Set preserves small colimits. Hence, the proof follows.

(3) We will show $f_{*}$ is a local surjection of sheaves. Then (1) and (2) will imply the result. Let $A \in \mathrm{M}$ and $g \in \mathbf{y}(C)(A)=\operatorname{hom}(A, C)$. By axioms of a pretopology, the pullback

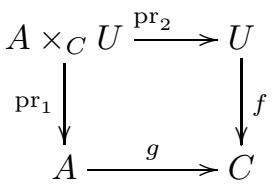


is a cover of $A$. Since $\operatorname{pr}_{2} \in \mathbf{y}(U)\left(A \times_{C} U\right)$, we can apply $f_{*}$ :

$$
f_{*}\left(\operatorname{pr}_{2}\right)=f \circ \operatorname{pr}_{2}=g \circ \operatorname{pr}_{1}=\operatorname{pr}_{1}^{*}(g) \in \mathbf{y}(C)\left(A \times_{C} U\right) .
$$

Hence, $f_{*}$ is a local surjection.

Now we will start connecting the homotopy theory of higher groupoids in $(M, \mathcal{T})$ with that of Kan simplicial sets. The next proposition says that a Kan fibration of higher groupoids is a "stalkwise Kan fibration" with respect to any collection of jointly conservative points.

Proposition 4.9. If $f: X \rightarrow Y$ is a Kan fibration of higher groupoids in $(\mathrm{M}, \mathcal{T})$ and $\mathcal{P}$ is a collection of jointly conservative points of $(\mathrm{M}, \mathcal{T})$, then for all $\mathrm{p} \in \mathcal{P}$ the map $\mathrm{p} f: \mathrm{p} X \rightarrow \mathrm{p} Y$ is a Kan fibration of simplicial sets.

Proof. Since $f: X \rightarrow Y$ is a Kan fibration, the horn projections

$$
\operatorname{Hom}\left(\Delta^{n}, X\right) \stackrel{\left(\iota_{n, j}^{*}, f_{*}\right)}{\longrightarrow} \operatorname{Hom}\left(\Lambda_{j}^{n} \rightarrow \Delta^{n}, X \rightarrow Y\right)
$$

are covers for all $n \geq 1$ and $0 \leq j \leq n$. Hence, Prop. 4.8 implies that for each point $p \in \mathcal{P}$

$$
\mathrm{p} \operatorname{Hom}\left(\Delta^{n}, X\right) \stackrel{\mathrm{p}\left(\iota_{n, j}^{*}, f_{*}\right)}{\longrightarrow} \mathrm{p} \operatorname{Hom}\left(\Lambda_{j}^{n} \rightarrow \Delta^{n}, X \rightarrow Y\right)
$$

is a surjection. Corollary 4.6 implies that $\mathrm{p}\left(\operatorname{Hom}\left(\Delta^{n}, X\right)\right) \cong \operatorname{hom}_{s \text { Set }}\left(\Delta^{n}, \mathrm{p} X\right)$, and since each $\mathrm{p}$ preserves finite limits, we have

$$
\mathrm{p}\left(\operatorname{Hom}\left(\Lambda_{j}^{n} \rightarrow \Delta^{n}, X \rightarrow Y\right)\right) \cong \operatorname{hom}_{s \operatorname{Set}}\left(\Lambda_{j}^{n}, \mathrm{p} X\right) \times_{\operatorname{hom}_{s \operatorname{Set}}\left(\Lambda_{j}^{n}, \mathrm{p} Y\right)} \operatorname{hom}_{s \operatorname{Set}}\left(\Delta^{n}, \mathrm{p} Y\right) .
$$

Combining these natural isomorphisms with (4.7) implies that $\mathrm{p} X \stackrel{\mathrm{p} f}{\longrightarrow} \mathrm{p} Y$ is a Kan fibration of simplicial sets.

Corollary 4.10. If $X$ is a higher groupoid in $(\mathrm{M}, \mathcal{T})$ and $\mathcal{P}$ is a collection of jointly conservative points for $(\mathrm{M}, \mathcal{T})$, then the simplicial set $\mathrm{p} X$ is a Kan complex for each $\mathrm{p} \in \mathcal{P}$.

\section{Stalkwise weak equivalences}

In this section, we introduce the morphisms between higher groupoids which will eventually become the weak equivalences in an incomplete category of fibrant objects. These stalkwise weak equivalences are a natural choice for weak equivalences between simplicial sheaves in diffeo-geometric contexts, e.g. [11, Def. 3.4.6].

Definition 5.1. Let $(M, \mathcal{T})$ be a category equipped with a pretopology and a collection of jointly conservative points $\mathcal{P}$. A morphism $f: X \rightarrow Y$ of higher groupoids in $(\mathrm{M}, \mathcal{T})$ is a stalkwise weak equivalence iff $\mathrm{p} f: \mathrm{p} X \rightarrow \mathrm{p} Y$ is a weak homotopy equivalence of simplicial sets for all $p \in \mathcal{P}$.

5.1. Simplicial homotopy groups for higher groups. Following Joyal [21], Henriques gave a definition of simplicial homotopy group sheaves for $n$-groups in a category equipped with a pretopology. (See also related work of Jardine [20].) We show here that a morphism $f: X \rightarrow Y$ of $n$-groups which induces an isomorphism between the simplicial homotopy groups is a stalkwise weak equivalence. We will need this result for integrating $L_{\infty}$ quasi-isomorphisms in Section 9. 
Let $S^{n}:=\Delta[n] / \partial \Delta[n]$ be the simplicial $n$-sphere, and let $\operatorname{cyl}\left(S^{n}\right)$ denote the simplicial set

$$
\operatorname{cyl}\left(S^{n}\right):=\Delta[n+1] /\left(\bigcup_{i=2}^{n+1} F^{i} \cup\left(F^{0} \cap F^{1}\right)\right)
$$

where $F^{i}$ is the simplicial set generated by the $i$ th face of $\Delta[n+1]$. There are two inclusions $i_{0}, i_{1}: S^{n} \rightarrow \operatorname{cyl}\left(S^{n}\right)$ induced by the maps $\Delta[n] \rightarrow F^{0}$ and $\Delta[n] \rightarrow F^{1}$, which are homotopy equivalences as well as homotopic to one another. Let $X$ be a reduced Kan simplicial set, i.e. $X_{0}=*$. Since we have a unique basepoint, for $n \geq 1$ we can define the $n$ th-homotopy group of $X$ as the coequalizer

$$
\pi_{n}(X)=\operatorname{coeq}\left(\operatorname{hom}_{s \mathrm{Set}}\left(\operatorname{cyl}\left(S^{n}\right), X\right) \rightrightarrows \operatorname{hom}_{s \mathrm{Set}}\left(S^{n}, X\right)\right) .
$$

In [17], Eq. (5.2) is used to provide an analogous definition of simplicial homotopy groups for higher groups in $(\mathrm{M}, \mathcal{T})$. The suitable analog of $\operatorname{hom}_{s \mathrm{Set}}(K,-)$ in $(5.2)$ is the matching object described in Sec. 4.2.

Definition 5.2 (Def. 3.1 [17]). Let $X$ be an $k$-group in (M, $\mathcal{T})$. Let $n \geq 1$ and let $S^{n}$ be the simplicial $n$-sphere, and $\operatorname{cyl}\left(S^{n}\right)$ the simplicial set (5.1). The simplicial homotopy groups $\pi_{n}^{\mathrm{spl}}(X)$ are the sheaves

$$
\pi_{n}^{\mathrm{spl}}(X):=\operatorname{coeq}\left(\operatorname{Hom}\left(\operatorname{cyl}\left(S^{n}\right), X\right) \rightrightarrows \operatorname{Hom}\left(S^{n}, X\right)\right),
$$

where $\operatorname{Hom}(-, X)$ is the sheaf (4.5).

Proposition 5.3. Let $X$ be an n-group in $(\mathrm{M}, \mathcal{T})$. Then for all points $\mathrm{p}: \mathrm{Sh}(\mathrm{M}) \rightarrow$ Set, there is a unique natural isomorphism of groups

$$
\phi_{X}: \mathrm{p} \pi_{*}^{\mathrm{spl}}(X) \stackrel{\cong}{\rightarrow} \pi_{*}(\mathrm{p} X)
$$

Proof. Since p preserves colimits, we have a natural isomorphism

$$
\mathrm{p} \pi_{k}^{\mathrm{spl}}(X) \cong \operatorname{coeq}\left(\mathrm{p} \operatorname{Hom}\left(\operatorname{cyl}\left(S^{k}\right), X\right) \rightrightarrows \mathrm{p} \operatorname{Hom}\left(S^{k}, X\right)\right) .
$$

Since $S^{n}$ and $\operatorname{cyl}\left(S^{n}\right)$ are finitely generated, Cor. 4.6 implies that there are natural isomorphisms

$$
\begin{aligned}
& \mathrm{pHom}\left(S^{n}, X\right) \cong \operatorname{hom}_{s \mathrm{Set}}\left(S^{n}, \mathrm{p} X\right), \\
& \mathrm{p} \operatorname{Hom}\left(\operatorname{cyl}\left(S^{n}\right), X\right) \cong \operatorname{hom}_{s \text { Set }}\left(\operatorname{cyl}\left(S^{n}\right), \mathrm{p} X\right) .
\end{aligned}
$$

Combining these natural isomorphisms and using the fact that $\operatorname{Hom}(-, X)$ is functorial (Prop. 4.5), we obtain a natural isomorphism

$$
\phi_{X}: \mathrm{p} \pi_{k}^{\mathrm{spl}}(X) \stackrel{\cong}{\rightarrow} \operatorname{coeq}\left(\operatorname{hom}_{s \operatorname{Set}}\left(\operatorname{cyl}\left(S^{k}\right), \mathrm{p} X\right) \rightrightarrows \operatorname{hom}_{s} \operatorname{Set}\left(S^{k}, \mathrm{p} X\right)\right)=\pi_{k}(\mathrm{p} X) .
$$

5.2. Stalkwise acyclic fibrations. Recall that an acyclic fibration of simplicial sets is a morphism of simplicial sets which is both a weak equivalence and a fibration. Equivalently, $X \rightarrow Y$ is an acyclic fibration of simplicial sets iff the boundary projections

$$
\operatorname{hom}_{s \text { Set }}\left(\Delta^{n}, X\right) \rightarrow \operatorname{hom}_{s \text { Set }}\left(\partial \Delta^{n}, X\right) \times_{\operatorname{hom}_{s \operatorname{Set}}\left(\partial \Delta^{n}, Y\right)} \operatorname{hom}_{s \mathrm{Set}}\left(\Delta^{n}, Y\right)
$$

are surjective for all $n \geq 0$. In Prop. 4.9 we showed that a Kan fibration $X \rightarrow Y$ between higher groupoids in $(\mathrm{M}, \mathcal{T})$ equipped with a jointly conservative collection 
of points $\mathcal{P}$ is a stalkwise Kan fibration, i.e. induces a Kan fibration $\mathrm{p} X \rightarrow \mathrm{p} Y$ for all $\mathrm{p} \in \mathcal{P}$. So clearly a Kan fibration which is also a stalkwise weak equivalence is obviously a "stalkwise acyclic fibration". In fact, more is true, as the following proposition shows:

Proposition 5.4. Let $(\mathrm{M}, \mathcal{T}, \mathcal{P})$ be a category equipped with a pretopology and a collection of jointly conservative points. A morphism $f: X \rightarrow Y$ of higher groupoids in $(\mathrm{M}, \mathcal{T})$ is both a stalkwise Kan fibration and a stalkwise weak equivalence (i.e., a stalkwise acyclic fibration) if and only if the boundary projections

$$
\operatorname{Hom}\left(\Delta^{n}, X\right) \stackrel{\left(\jmath_{n}^{*}, f_{*}\right)}{\longrightarrow} \operatorname{Hom}\left(\partial \Delta^{n} \stackrel{\jmath_{n}}{\longrightarrow} \Delta^{n}, X \stackrel{f}{\rightarrow} Y\right)
$$

are stalkwise surjections for $n \geq 0$.

Proof. Recall that the sheaf $\operatorname{Hom}\left(\partial \Delta^{n} \rightarrow \Delta^{n}, X \rightarrow Y\right)(3.2)$ is the pullback

$$
\operatorname{Hom}\left(\partial \Delta^{n}, X\right) \times_{\operatorname{Hom}\left(\partial \Delta^{n}, Y\right)} \operatorname{Hom}\left(\Delta^{n}, Y\right)
$$

Corollary 4.6 implies that $\mathrm{p}\left(\operatorname{Hom}\left(\Delta^{n}, X\right)\right) \cong \operatorname{hom}_{s \text { Set }}\left(\Delta^{n}, \mathrm{p} X\right)$ for all $\mathrm{p} \in \mathcal{P}$, and since each $\mathrm{p}$ preserves finite limits, we have

$$
\begin{aligned}
\mathrm{p}\left(\operatorname{Hom}\left(\partial \Delta^{n}, X\right)\right. & \left.\times_{\operatorname{Hom}\left(\partial \Delta^{n}, Y\right)} \operatorname{Hom}\left(\Delta^{n}, Y\right)\right) \\
& \cong \operatorname{hom}_{s \operatorname{Set}}\left(\partial \Delta^{n}, \mathrm{p} X\right) \times_{\operatorname{hom}_{s \text { Set }}\left(\partial \Delta^{n}, \mathrm{p} Y\right)} \operatorname{hom}_{s \text { Set }}\left(\Delta^{n}, \mathrm{p} Y\right) .
\end{aligned}
$$

Hence, by comparison with (5.3), we see that $\mathrm{p} f: \mathrm{p} X \rightarrow \mathrm{p} Y$ is an acyclic fibration of simplicial sets if and only if $\mathrm{p}\left(\jmath_{n}^{*}, f_{*}\right)$ is surjective, i.e. $\left(f_{n}^{*}, f_{*}\right)$ is a stalkwise surjection.

\section{HYPERCOVERS}

Hypercovers were introduced in [2] and subsequently have been used throughout the homotopy theory of simplicial sheaves, e.g. [5]. Hypercovers for Lie $n$-groupoids play an important role in the work of Zhu [43] and Wolfson [42]. Also, the acyclic fibrations in the Behrend-Getzler CFO structure for $n$-groupoids objects in a descent category are hypercovers.

Definition 6.1. Given a category and pretopology $(\mathrm{M}, \mathcal{T})$, a morphism $f: X \rightarrow Y$ of simplicial objects in $\mathrm{M}$ is a hypercover iff it satisfies the condition $\operatorname{Acyc}(m)$ for all $0 \leq m$, which means the sheaf $\operatorname{Hom}\left(\partial \Delta^{m} \rightarrow \Delta^{m}, X \rightarrow Y\right)$ is representable and the canonical boundary projection:

$$
\operatorname{Hom}\left(\Delta^{m}, X\right) \stackrel{q_{m}:=\left(\jmath_{m}^{*}, f_{*}\right)}{\longrightarrow} \operatorname{Hom}\left(\partial \Delta^{m} \stackrel{\jmath_{m}}{\longrightarrow} \Delta^{m}, X \stackrel{f}{\rightarrow} Y\right)
$$

is a cover in $\mathcal{T}$. For $m=0, \operatorname{Hom}\left(\partial \Delta^{0} \rightarrow \Delta^{0}, X \rightarrow Y\right):=Y_{0}$ by definition.

Remark 6.2.

(1) As shown in [43, Lemma 2.4], if $Y$ is a higher groupoid in $(\mathrm{M}, \mathcal{T})$, and $f: X \rightarrow Y$ satisfies (6.1) for $l<m$, then $\operatorname{Hom}\left(\partial \Delta^{m} \stackrel{\jmath_{m}}{\rightarrow} \Delta^{m}, X \stackrel{f}{\rightarrow} Y\right)$ is automatically representable.

(2) A hypercover is a Kan fibration automatically, since maps in $\left\{\Lambda_{j}^{m} \rightarrow \Delta^{m} \mid\right.$ $m>0,0 \leq j \leq m\}$ can be reconstructed as pushouts of ones in $\left\{\partial \Delta^{m} \rightarrow\right.$ $\left.\Delta^{m} \mid m \geq 0\right\}$.

The $\operatorname{Acyc}(m)$ condition is obviously analogous to the previously discussed characterization (5.3) of acyclic fibrations of simplicial sets. Indeed, every hypercover of higher groupoids is a stalkwise acyclic fibration in the sense of Prop. 5.4: 
Corollary 6.3. If a morphism of higher groupoids $f: X \rightarrow Y$ in $(\mathrm{M}, \mathcal{T}, \mathcal{P})$ is a hypercover, then it is Kan fibration and a stalkwise weak equivalence.

Proof. Remark 6.2 implies that $f: X \rightarrow Y$ is a Kan fibration. Since the boundary projections (6.1) are covers, Prop. 4.8 implies that they are stalkwise surjections with respect to the points $\mathcal{P}$. Hence, Prop. 5.4 implies that $f: X \rightarrow Y$ is a stalkwise acyclic fibration, so in particular, it is a stalkwise weak equivalence.

The natural question to ask is whether the converse of Cor. 6.3 is true. That is, is every morphism of higher groupoids which is both a Kan fibration and a stalkwise weak equivalence necessarily a hypercover? The answer will be yes, if our pretopology satisfies some additional conditions. Our main example, the category Mfd of Banach manifolds equipped with the surjective submersion pretopology $\mathcal{T}_{\text {subm }}$ and the collection of points $\mathcal{P}_{\text {Ban }}$, satisfies these additional conditions.

\subsection{Locally stalkwise pretopologies.}

Definition 6.4. Let $(M, \mathcal{T}, \mathcal{P})$ be a category equipped with a pretopology and a jointly conservative collection of points. Let $X \in \mathrm{M}$. A morphism $F \stackrel{g}{\rightarrow} \mathbf{y} X$ of sheaves is a local stalkwise cover iff there exists an object $Y \in \mathrm{M}$ and a stalkwise surjection (Def. 4.7) $\mathbf{y} Y \stackrel{f}{\rightarrow} F$ such that the composition $g \circ f: \mathbf{y} Y \rightarrow \mathbf{y} X$ is a cover in $(\mathrm{M}, \mathcal{T})$.

Definition 6.5. A pretopology $\mathcal{T}$ on a category $M$ is a locally stalkwise pretopology iff there exists a jointly conservative collection of points $\mathcal{P}$ of $(\mathrm{M}, \mathcal{T})$ such that:

(1) (2-out-of-3) If $U \stackrel{f}{\rightarrow} V \stackrel{g}{\rightarrow} W$ are morphisms in $\mathrm{M}$, and $g \circ f$ is a cover and $\mathbf{y}(f)$ is a stalkwise surjection in $\operatorname{Sh}(\mathrm{M})$ with respect to $\mathcal{P}$, then $g$ is also a cover,

(2) (locality of covers) If $W \stackrel{q}{\rightarrow} V$ and $U \stackrel{p}{\rightarrow} V$ are morphisms in $\mathrm{M}, \mathbf{y}(q)$ is a stalkwise surjection with respect to $\mathcal{P}$, and the base change $U \times_{V} W \stackrel{\tilde{p}}{\rightarrow} W$ is a local stalkwise cover, then $p$ is a cover, and therefore $U \times_{V} W$ is representable.

We have several remarks about Def. 6.5:

Remark 6.6.

(1) It is not really precise to call the first condition in Def. 6.5: "2-out-of-3", because $g \circ f$ being a cover and $g$ being a cover will not imply anything (just like surjective maps for sets). We note that our "2-out-of-3" property holds automatically for stalkwise surjections in $\mathrm{Sh}(\mathrm{M})$.

(2) If $(M, \mathcal{T}, \mathcal{P})$ is a category equipped with a locally stalkwise pretopology then a local stalkwise cover $F \stackrel{g}{\rightarrow} \mathbf{y} X$ of $X$ is a cover in $\mathcal{T}$ whenever $F$ is representable.

We now can give a converse to Cor. 6.3. In fact, we have:

Proposition 6.7. Let $(\mathrm{M}, \mathcal{T})$ be a category equipped with a locally stalkwise pretopology with respect to a jointly conservative collection of points $\mathcal{P}$, and let $f: X \rightarrow$ $Y$ be a morphism of higher groupoids in $(\mathrm{M}, \mathcal{T})$. The following are equivalent: 
(1) $f: X \rightarrow Y$ is a Kan fibration and a stalkwise weak equivalence with respect to the collection of points $\mathcal{P}$.

(2) $f: X \rightarrow Y$ is a Kan fibration and a stalkwise weak equivalence with respect to any jointly conservative collection of points of $(\mathrm{M}, \mathcal{T})$.

(3) $f: X \rightarrow Y$ is a hypercover.

Proof. (3) $\Rightarrow(2)$ is the content of Cor. 6.3, and (2) $\Rightarrow(1)$ is obvious. So we focus on $(1) \Rightarrow(3)$.

First, let $k \geq 1$ and consider the following pullback diagram in $\mathrm{Sh}(\mathrm{M})$ (6.2)

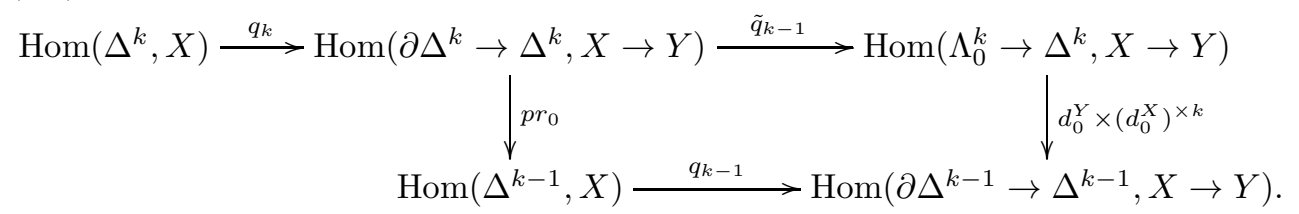

The projection $\operatorname{Hom}\left(\partial \Delta^{k} \rightarrow \Delta^{k}, X \rightarrow Y\right) \rightarrow \operatorname{Hom}\left(\Delta^{k-1}, X\right)=X_{k-1}$ is induced by $d_{0}^{X}$. The composition $\tilde{q}_{k-1} \circ q_{k}: \operatorname{Hom}\left(\Delta^{k}, X\right) \rightarrow \operatorname{Hom}\left(\Lambda_{0}^{k} \rightarrow \Delta^{k}, X \rightarrow Y\right)$ is the horn projection, and therefore a cover, since $f: X \rightarrow Y$ is a Kan fibration. Since $f$ is also a stalkwise weak equivalence, Prop. 5.4 implies that $q_{k}$ is a stalkwise surjection. Hence, $\tilde{q}_{k-1}$ is a local stalkwise cover (Def. 6.4).

Next, we observe that the composition $p r_{0} \circ q_{k}: \operatorname{Hom}\left(\Delta^{k}, X\right) \rightarrow \operatorname{Hom}\left(\Delta^{k-1}, X\right)$ is simply the face map $d_{0}: X_{k} \rightarrow X_{k-1}$, which is a cover (Lemmas 3.6 and 3.7), hence a stalkwise surjection (Lemma 4.8). Combining this with the fact that $q_{k}$ is a stalkwise surjection implies that $p r_{0}$ is a local stalkwise cover, and hence also a stalkwise surjection. Thus $d_{0}^{Y} \times\left(d_{0}^{X}\right)^{k} \circ \tilde{q}_{k-1}=q_{k-1} \circ p r_{0}$ is stalkwise surjective, hence $d_{0}^{Y} \times\left(d_{0}^{X}\right)^{k}$ is stalkwise surjective.

Now, we show via induction that the boundary maps $\operatorname{Hom}\left(\Delta^{k}, X\right) \stackrel{q_{k}}{\longrightarrow} \operatorname{Hom}\left(\partial \Delta^{k} \rightarrow\right.$ $\left.\Delta^{k}, X \rightarrow Y\right)$ are covers for all $k \geq 0$. For the base case, let $k=1$. Then Def. 6.1 implies that $\operatorname{Hom}\left(\partial \Delta^{k-1} \rightarrow \Delta^{k-1}, X \rightarrow Y\right)=Y_{0}$ is representable, and since $f$ is a Kan fibration, $\operatorname{Hom}\left(\Lambda_{0}^{1} \rightarrow \Delta^{1}, X \rightarrow Y\right)$ is also representable. The results of the previous paragraphs imply that $\tilde{q}_{0}$ is a local stalkwise cover and $d_{0}^{Y} \times\left(d_{0}^{X}\right)^{k}$ is a stalkwise surjection. Since $\mathcal{T}$ is a locally stalkwise pretopology, Def. 6.5 (2) implies that $q_{0}$ is a cover and $\operatorname{Hom}\left(\partial \Delta^{1} \rightarrow \Delta^{1}, X \rightarrow Y\right)$ is representable.

Finally, suppose $k \geq 2, \operatorname{Hom}\left(\Delta^{k-2}, X\right) \stackrel{q_{k-2}}{\longrightarrow} \operatorname{Hom}\left(\partial \Delta^{k-2} \rightarrow \Delta^{k-2}, X \rightarrow Y\right)$ is a cover, and $\operatorname{Hom}\left(\partial \Delta^{k-1} \rightarrow \Delta^{k-1}, X \rightarrow Y\right)$ is representable. As previously shown, the pullback of $q_{k-1}$ in diagram (6.2) along the stalkwise surjection $d_{0}^{Y} \times\left(d_{0}^{X}\right)^{k}$ is a local stalkwise cover. Hence, Def. 6.5(2) implies that $q_{k-1}$ : $\operatorname{Hom}\left(\Delta^{k-2}, X\right) \rightarrow$ $\operatorname{Hom}\left(\partial \Delta^{k-1} \rightarrow \Delta^{k-1}, X \rightarrow Y\right)$ is a cover, and that $\operatorname{Hom}\left(\partial \Delta^{k} \rightarrow \Delta^{k}, X \rightarrow Y\right)$ is representable. This completes the proof.

6.2. Locally stalkwise pretopologies for Banach manifolds. Here we show that the pretopology $\mathcal{T}_{\text {subm }}$ of surjective submersions equipped with the collection of jointly conservative points $\mathcal{P}_{\text {Ban }}$ (4.1) is a locally stalkwise pretopology for the category of Banach manifolds. We first give an explicit description of stalkwise surjective maps in $\left(\mathrm{Mfd}, \mathcal{T}_{\text {subm }}, \mathcal{P}_{\text {Ban }}\right)$.

Lemma 6.8. Let $X \stackrel{f}{\rightarrow} Y \stackrel{g}{\rightarrow} Z$ be composable morphisms in $\mathrm{Mfd}$. If $f$ is surjective and $g \circ f$ is a surjective submersion, then $g$ is also a surjective submersion. 
Proof. Clearly, $g$ is surjective. Let $y \in Y, z=g(y)$, and $x \in f^{-1}(y)$. Since $g \circ f$ is a surjective submersion, there exists open neighborhoods $U$ and $V$ of $z$ and $x$, respectively, and a section $\sigma_{X Z}: U \rightarrow V$ such that $\sigma_{X Z}(z)=x$, and $g \circ f \circ \sigma_{X Z}=$ $\operatorname{id}_{U}$. Let $W=g^{-1}(U)$. Then $\sigma:=f \circ \sigma_{X Z}: U \rightarrow W$ is a section of $g$ such that $\sigma(z)=y$. Hence $g$ is a surjective submersion.

Lemma 6.9. Let $\phi: X \rightarrow Y$ be a morphism in (Mfd, $\left.\mathcal{T}_{\text {subm }}, \mathcal{P}_{\text {Ban }}\right)$. Then the following are equivalent:

(1) The morphism of sheaves $\mathbf{y}(\phi): \mathbf{y} X \rightarrow \mathbf{y} Y$ is stalkwise surjective.

(2) For any morphism $f: U \rightarrow Y$ in Mfd, there exists an open cover $\left\{U_{i}\right\}_{i \in \mathcal{I}}$ of $U$ and morphisms $f_{i}: U_{i} \rightarrow X$ such that $\varphi \circ f_{i}=\left.f\right|_{U_{i}}$ for each $i \in \mathcal{I}$.

(3) For each $y \in Y$, there exists: a preimage $x \in \phi^{-1}(y)$, an open neighborhood of $V$ of $y$, an open neighborhood of $W$ of $x$, and a morphism $\sigma: V \rightarrow W$ such that $\sigma(y)=x$ and $\phi \circ \sigma=\mathrm{id}_{V}$.

Proof.

$(1 \Rightarrow 2)$ : Let $f: U \rightarrow Y$ be a morphism of Banach manifolds. Since $\phi$ is stalkwise surjective, for each $z \in U$, we can find a Banach space $V_{z}$, an $r_{z}>0$ and a local diffeomorphism $i_{z}: B_{V_{z}}\left(r_{z}\right) \rightarrow U$ with $i_{z}(0)=z$ such that there exists a map $f_{z}: B_{V_{z}}\left(r_{z}\right) \rightarrow X$ with the property that $\phi \circ f_{z}=f \circ i_{z}$. We then use collection of open balls $\left\{B_{V_{z}}\left(r_{z}\right)\right\}_{z \in U}$ to obtain the desired open cover of $U$, and the collection $\left\{f_{z}\right\}_{z \in U}$ as our desired collection of maps.

$(2 \Rightarrow 1)$ : Let $V$ be a Banach space and $\bar{g} \in p_{V} \mathbf{y} Y$. This class is represented by a map $g: B_{V}(r) \rightarrow Y$. By hypothesis, there exists an open cover $\left\{U_{i}\right\}$ of $B_{V}(r)$ and maps $h_{i}: U_{i} \rightarrow X$ such that $\varphi \circ h_{i}=\left.g\right|_{U_{i}}$. Let $U_{i_{0}}$ be an open subset containing 0 , and $r^{\prime}>0$ such that $B_{V}\left(r^{\prime}\right) \subseteq U_{i_{0}}$. Then $\left.g\right|_{B_{V}\left(r^{\prime}\right)}$ is the composition of $\varphi$ with $f=\left.h_{i_{0}}\right|_{B_{V}\left(r^{\prime}\right)}$, and hence $p_{V} \varphi(\bar{f})=\bar{g}$.

$(2 \Rightarrow 3)$ : Let $y \in Y$ and consider the identity map $\operatorname{id}_{Y}: Y \rightarrow Y$. There there exists an open cover $\left\{U_{i}\right\}$ of $Y$ and maps $f_{i}: U_{i} \rightarrow Y$ such that $\varphi \circ f_{i}=\operatorname{id} U_{U_{i}}$. Let $U_{i^{\prime}}$ be an element of the cover containing $y$, and let $x=f_{i^{\prime}}(y)$. Then $\sigma:=f_{i^{\prime}}: U_{i^{\prime}} \rightarrow X$ is a desired local section of $\varphi$ which maps $y$ to $x$.

$(3 \Rightarrow 2)$ : Let $f: U \rightarrow Y$ be a map. For each $y \in Y$, there exists an $x \in \phi^{-1}(y)$, an open subset $V_{y}$ containing $y$, and open subset $W_{y}$ containing $x$ and a local section $\sigma_{y}: V_{y} \rightarrow W_{y}$ mapping $y$ to $x$. The collection $\left\{U_{y}:=f^{-1}\left(V_{y}\right)\right\}_{y \in Y}$ is an open cover of $U$. For each $y \in Y$, let $f_{y}: U_{y} \rightarrow X$ be the composition $f_{y}=\left.\sigma_{y} \circ f\right|_{U_{y}}$. Then, by construction, $\varphi \circ f_{y}=\left.f\right|_{U_{y}}$.

Remark 6.10. Note that Lemma 6.9 implies that stalkwise surjective maps are weaker than surjective submersions. For example, given a point $x \in X$, it is easy to see, by item (2) above, the natural map $X \sqcup\{x\} \rightarrow X$ is a stalkwise surjective map but not a surjective submersion. Also note that Remark 4.3 implies that Lemma 6.9 also holds if we replace $\mathcal{T}_{\text {ss }}$ with $\mathcal{T}_{\text {open }}$.

Lemma 6.11. Let $W \stackrel{q}{\rightarrow} V \stackrel{p}{\leftarrow} U$ be morphisms in Mfd such that $\mathbf{y}(q)$ is stalkwise surjective, and consider the pullback diagram in the category of topological spaces

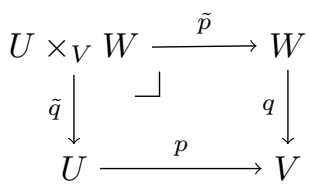


Suppose that there exists a manifold $X \in \mathrm{Mfd}$ and a continuous surjective map $X \stackrel{\pi}{\rightarrow} U \times_{V} W$ such that $\tilde{q} \circ \pi: X \rightarrow U$ is smooth and the composition $\tilde{p} \circ \pi: X \rightarrow W$ is a smooth surjective submersion. Then $p: U \rightarrow V$ is a surjective submersion and therefore $U \times_{V} W$ is representable in Mfd.

Proof. The surjectivity of $p$ follows from an easy set-theoretical argument, so we will show it is a surjective submersion. Let $u \in U$ and $v=p(u)$. Since $\mathbf{y}(q)$ is stalkwise surjective, Lemma 6.9 implies there exists an open neighborhood $O_{v}$ of $v$, an element $w \in q^{-1}(v) \subseteq W$, and a smooth section

$$
\sigma_{W V}: O_{v} \rightarrow O_{w}:=q^{-1}\left(O_{v}\right)
$$

such that $\sigma_{W V}(v)=w, \quad q \circ \sigma_{W V}=\operatorname{id}_{O_{v}}$. Hence the restriction $\left.q\right|_{O_{w}}$ is a submersion at $w$ (i.e., $T_{w} q$ is surjective and its kernel splits). The inverse function theorem [25, Cor. I.5.2s] then implies that, by taking $O_{v}$ to be a small enough neighborhood, we can express $\left.q\right|_{O_{w}}$ as a projection. Therefore, the following pullback diagram exists in the category Mfd:

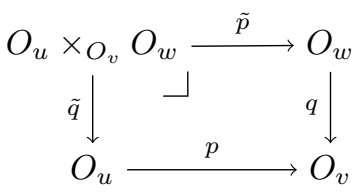

where $O_{u}:=p^{-1}\left(O_{v}\right)$, and we suppress restrictions of the morphisms.

Now, by hypothesis, we have a surjective continuous map $\pi: X \rightarrow U \times{ }_{V} W$ such that $\pi \circ \tilde{q}$ is smooth and $\pi \circ \tilde{p}$ is a surjective submersion. Let $x \in X$ such that $\pi(x)=(u, w)$. We may shrink $O_{v}$ further if necessary, so that we have a smooth section

$$
\sigma_{X W}: O_{w} \rightarrow O_{x}:=(\tilde{p} \circ \pi)^{-1}\left(O_{w}\right)
$$

such that $(\tilde{p} \circ \pi) \circ \sigma_{X W}=\operatorname{id}_{O_{w}}$ and $\sigma_{X W}(w)=x$. It is not difficult to see that we have the following equalities of open sets:

$$
\tilde{p}^{-1}\left(O_{w}\right)=O_{u} \times O_{v} O_{w}, \quad \pi^{-1}\left(O_{u} \times_{O_{v}} O_{w}\right)=O_{x}
$$

Since $\tilde{p} \circ \pi \circ \sigma_{X W}=\operatorname{id}_{O_{w}}$, we see that

$$
\pi \circ \sigma_{X W}: O_{w} \rightarrow O_{u} \times O_{v} O_{w}
$$

is a continuous section of $\tilde{p}: O_{u} \times{ }_{O_{v}} O_{w} \rightarrow O_{w}$. In fact, $\pi \circ \sigma_{X W}$ is smooth since the factors $\tilde{q} \circ \pi \circ \sigma_{X W}$ and $\tilde{p} \circ \pi \circ \sigma_{X W}$ are compositions of smooth maps.

The commutativity of the pushout diagram gives us $p \circ \tilde{q} \circ \pi \circ \sigma_{X W}=q$. Hence,

$$
\tilde{q} \circ \pi \circ \sigma_{X W} \circ \sigma_{W V}: O_{v} \rightarrow O_{u}
$$

is our desired smooth section of $p$. Therefore $p$ is a submersion.

Proposition 6.12. The category of Banach manifolds $\mathrm{Mfd}$ equipped with the surjective submersion pretopology $\mathcal{T}_{\text {subm, }}$, and the collection of jointly conservative points $\mathcal{P}_{\mathrm{Ban}}$ is a locally stalkwise pretopology.

Proof. First, we show the "2-out-of-3" property of Def. 6.5 is satisfied. Indeed, this follows immediately from Lemma 6.8 , since if $\mathbf{y}(f): \mathbf{y}(X) \rightarrow \mathbf{y}(Y)$ is stalkwise surjective with respect to $\mathcal{P}_{\mathrm{Ban}}$ then $f: X \rightarrow Y$ is surjective. 
Next, we show that the locality property of Def. 6.5 is satisfied. Let $W \stackrel{q}{\rightarrow}$ $V \stackrel{p}{\leftarrow} U$ be morphisms in Mfd and suppose $\mathbf{y} q: \mathbf{y} W \rightarrow \mathbf{y} V$ is a stalkwise surjection. Furthermore, suppose we have a diagram of sheaves

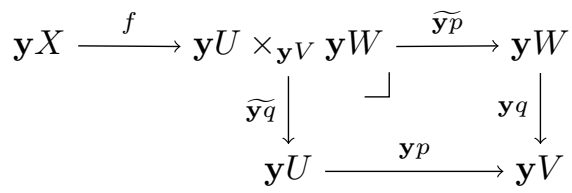

which exhibits the pullback $\widetilde{\mathbf{y} p}$ as a local stalkwise cover, i.e. $f$ is a stalkwise surjection, and $\widetilde{\mathbf{y p}} \circ f$ is represented by a surjective submersion $g: X \rightarrow W$. Note that the Yoneda lemma implies that there exists a smooth map $h: X \rightarrow U$ representing the composition $\widetilde{\mathbf{y} q} \circ f$. Moreover, since $f$ is stalkwise surjective, it is represented in the category of topological spaces by a surjective map $\pi=(h, g): X \rightarrow U \times_{V} W$. This observation, combined with the fact that $g$ is a surjective submersion, implies that the hypotheses of Lemma 6.11 are satisfied, and therefore we conclude that $p: U \rightarrow V$ is a cover.

\section{Higher groupoids as AN InCOMPlete CATEGORY OF FIBRANT OBJECTS}

We now fix a category $(M, \mathcal{T}, \mathcal{P})$ equipped with a locally stalkwise pretopology and prove that the corresponding category of $\infty$-groupoids admits an iCFO structure. The results in Sec. 6.2 then imply that we obtain an iCFO structure for the category of Lie $\infty$-groupoids as a special case.

After discussing some aspects of the simplicial localization of category of Lie $\infty$ groupoids in Sec. 7.2.1, we analyze in Sec. 7.3 the weak equivalences for this iCFO structure in more detail. In particular, we recall that the weak equivalences are completely characterized by the acyclic fibrations, which in this case are, respectively, stalkwise weak equivalences and hypercovers. Thanks to a result of Behrend and Getzler [3], we obtain a sheaf-theoretic independent characterization of weak equivalences, without any mention of the collection of points $\mathcal{P}$.

7.1. Path object. We first construct a candidate for a path object. This will require several steps. Later in Sec. 7.2, we verify that this gives a path object as part of the iCFO structure for higher groupoids. Our construction, in particular the proof of Prop. 7.2, is essentially identical to that of Behrend and Getzler [3, Thm. 3.21]. However, as previously mentioned, we do not assume the existence of finite limits in our category M. Moreover, our definition of weak equivalences, necessary for our application in Sec. 9 is different than the one given in [3]. Hence, it is necessary to present a verification that the Behrend-Getzler construction works in our context.

Given a simplicial sheaf $F \in s \operatorname{Sh}(\mathrm{M})$ and a simplicial set $K \in s$ Set, denote by $F^{K}$ the simplicial sheaf

$$
F^{K}(U)_{n}:=\operatorname{hom}_{s \text { Set }}\left(\Delta^{n} \times K, F(U)\right)=M_{\Delta^{n} \times K}(F),
$$

where $M_{\Delta^{n} \times K}(F)$ is the aforementioned matching object (Def. 4.4). A natural path object for $F$ is the simplicial sheaf $F^{\Delta^{1}}$. The inclusion of simplicial sets

$$
\partial \Delta^{1} \cong \Delta^{0} \cup \Delta^{0} \stackrel{d^{0} \cup d^{1}}{\longrightarrow} \Delta^{1}
$$


induces a morphism of simplicial sheaves

$$
F^{\Delta^{1}} \stackrel{\left(d^{0 *}, d^{1 *}\right)}{\longrightarrow} F \times F \cong F^{\Delta^{0} \cup \Delta^{0}} .
$$

Also, the constant map $s^{0}: \Delta^{1} \rightarrow \Delta^{0}$ gives us a map of simplicial sheaves

$$
F \cong F^{\Delta^{0}} \stackrel{s^{0 *}}{\longrightarrow} F^{\Delta^{1}}
$$

We now make the following observation which justifies our consideration of $F^{\Delta^{1}}$ :

Proposition 7.1. Let $X$ be a higher groupoid in $(\mathrm{M}, \mathcal{T}, \mathcal{P})$. In the diagram

$$
\mathbf{y} X \stackrel{s^{0 *}}{\longrightarrow}(\mathbf{y} X)^{\Delta^{1}} \stackrel{\left(d^{0 *}, d^{1 *}\right)}{\longrightarrow} \mathbf{y} X \times \mathbf{y} X .
$$

the map $s^{0 *}$ is a stalkwise weak equivalence and the map $\left(d^{0 *}, d^{1 *}\right)$ is a stalkwise Kan fibration.

Proof. Recall that Corollary 4.10 implies that $\mathrm{p} X:=\mathrm{py} X$ is a Kan complex for any point $\mathrm{p} \in \mathcal{P}$. Equation 7.1 and Prop. 4.5 imply that the image of the above diagram under $p$ is naturally isomorphic to the diagram of simplicial sets

$$
\mathrm{p} X \stackrel{s^{0 *}}{\longrightarrow}(\mathrm{p} X)^{\Delta^{1}} \stackrel{\left(d^{0 *}, d^{1 *}\right)}{\longrightarrow} \mathrm{p} X \times \mathrm{p} X .
$$

This is the usual diagram which exhibits the Kan complex $(\mathrm{p} X)^{\Delta^{1}}=\operatorname{Map}_{s \mathrm{Set}}\left(\Delta^{1}, \mathrm{p} X\right)$ as the path object of $\mathrm{p} X$. Hence, $s^{0 *}$ is a weak equivalence and $\left(\mathrm{p} d^{0 *}, \mathrm{p} d^{1 *}\right)$ is a fibration of simplicial sets for any point $\mathrm{p}$.

The above proposition suggests that, to construct a path object for an $n$-groupoid $X$ in $\mathrm{M}$, we should show that the simplicial sheaf $(\mathbf{y} X)^{\Delta^{1}}$ is representable by a higher groupoid $X^{\Delta^{1}}$, and that the map of higher groupoids $X^{\Delta^{1}} \rightarrow X \times X$ is not just a stalkwise Kan fibration, but a Kan fibration in the sense of Def. 3.3.

As a first step, we prove the following Proposition:

Proposition 7.2. If $X$ is an $\infty$-groupoid in $(\mathrm{M}, \mathcal{T})$, then there is a canonical $\infty$-groupoid $X^{\Delta^{1}}$ in $(\mathrm{M}, \mathcal{T})$ representing the simplicial sheaf $(\mathbf{y} X)^{\Delta^{1}}$.

We need the following Lemma whose proof we will postpone for the moment.

Lemma 7.3. The inclusion

$$
\Lambda_{j}^{n} \times \Delta^{1} \rightarrow \Delta^{n} \times \Delta^{1}
$$

is a collapsible extension (Def. 3.5).

Proof of Prop. 7.2. Let $X$ be an $\infty$-groupoid in M. Proposition 4.5 and (7.1) imply that

$$
(\mathbf{y} X)_{n}^{\Delta^{1}} \cong \operatorname{Hom}\left(\Delta^{n} \times \Delta^{1}, X\right)
$$

on each level $n$. It follows from the presentation of $\Delta^{n} \times \Delta^{1}$ (e.g., see Appendix A) that $\operatorname{Hom}\left(\Delta^{n} \times \Delta^{1}, X\right)$ is represented by

$$
X_{n+1} d_{1} \times_{d_{1}} X_{n+1} \quad d_{2} \times{ }_{d_{2}} \cdots_{d_{i}} \times_{d_{i}} X_{n+1} \quad d_{i+1} \times{ }_{d_{i+1}} \cdots_{d_{n}} \times_{d_{n}} X_{n+1} .
$$

Lemmas 3.6 and 3.7 imply that the face maps of $X$ are covers. Hence, the above pullbacks exist in $\mathrm{M}$. Therefore, $(\mathbf{y} X)^{\Delta^{1}}$ is represented by a simplicial object $X^{\Delta^{1}}$. 
Next we prove that $X^{\Delta^{1}}$ is an $\infty$-groupoid in M. By definition, we need to show the condition $\operatorname{Kan}(n, j)$ holds, i.e., $\operatorname{Hom}\left(\Lambda_{j}^{n}, X^{\Delta^{1}}\right)$ is representable and the map induced by the inclusion

$$
\operatorname{Hom}\left(\Delta^{n}, X^{\Delta^{1}}\right) \rightarrow \operatorname{Hom}\left(\Lambda_{j}^{n}, X^{\Delta^{1}}\right)
$$

is a cover, for all $n \geq 1$ and $0 \leq j \leq n$. Note that Prop. 4.5 implies that we have a natural isomorphism,

$$
\operatorname{Hom}\left(\Lambda_{j}^{n}, X^{\Delta^{1}}\right) \cong \operatorname{Hom}\left(\Lambda_{j}^{n} \times \Delta^{1}, X\right) .
$$

We proceed by induction. For the $n=1$ case, we have

$$
\operatorname{Hom}\left(\Delta^{1} \times \Delta^{1}, X\right) \rightarrow \operatorname{Hom}\left(\Lambda_{j}^{1} \times \Delta^{1}, X\right) \cong \operatorname{Hom}\left(\Delta^{1}, X\right) \cong X_{1} .
$$

Since $X$ is a higher groupoid, Lemma 7.3 combined with Lemma 3.7 imply that $\operatorname{Kan}(1, j)$ is satisfied.

Now assume $n>1$ and that $\operatorname{Kan}(m, j)$ holds for all $m<n$ and $0 \leq j \leq m$. This plus the fact that $\Lambda_{j}^{n}$ is a collapsible subset of $\Delta^{n}$, allows us to apply Lemma 3.9 to conclude that $\operatorname{Hom}\left(\Lambda_{j}^{n}, X^{\Delta^{1}}\right)$ is representable. Hence, Lemma 7.3 again combined with Lemma 3.7 imply that $\operatorname{Kan}(n, j)$ is satisfied. This completes the proof.

It remains to prove Lemma 7.3. We start with the following auxiliary Lemma:

Lemma 7.4. Let $f: \Lambda_{i}^{1} \rightarrow \Delta^{1}$, for either $i=0$ or $i=1$ be the usual inclusion. For every boundary extension $\iota: S \rightarrow T$ the induced map

$$
\left(S \times \Delta^{1}\right) \sqcup_{S \times \Lambda_{i}^{1}}\left(T \times \Lambda_{i}^{1}\right) \rightarrow T \times \Delta^{1},
$$

is a collapsible extension.

Proof. The case when $\iota: S \rightarrow T$ is the standard inclusion $\partial \Delta^{n} \rightarrow \Delta^{n}$ is proven in [19, Lemma 3.3.3]. We next observe the following following fact: If $F: s$ Set $\times s$ Set $\rightarrow$ $s$ Set is a co-continuous functor and

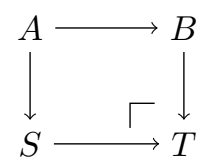

is a pushout square of simplicial sets, then the diagram

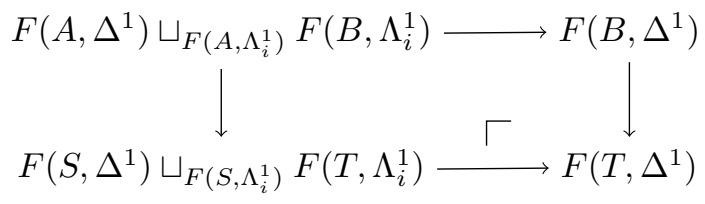

is again a pushout square. (See [26, Lemma 2.42], where $F$ is taken to be the join functor, and observe that only the co-continuity of the join is used in the proof.)

Hence, to prove the statement, we take $F$ above to be the product functor and proceed by induction.

Since any collapsible extension is a boundary extension, we have the following corollary: 
Corollary 7.5. Let $f: \Lambda_{i}^{1} \rightarrow \Delta^{1}$, for either $i=0$ or $i=1$ be the usual inclusion. For every collapsible extension $\iota: S \rightarrow T$ the induced map

$$
\left(S \times \Delta^{1}\right) \sqcup_{S \times \Lambda_{i}^{1}}\left(T \times \Lambda_{i}^{1}\right) \rightarrow T \times \Delta^{1},
$$

is a collapsible extension.

Proof of Lemma 7.3. We observe that the morphism $\Lambda_{j}^{n} \times \Delta^{1} \rightarrow \Delta^{n} \times \Delta^{1}$ is a composition of the following maps

$$
\Lambda_{j}^{n} \times \Delta^{1} \rightarrow\left(\Lambda_{j}^{n} \times \Delta^{1}\right) \sqcup_{\Lambda_{j}^{n} \times \Lambda_{i}^{1}}\left(\Delta^{n} \times \Lambda_{i}^{1}\right) \rightarrow \Delta^{n} \times \Delta^{1} .
$$

The first map is clearly a collapsible extension (note that $\Lambda_{i}^{1}=\Delta^{0}$ ). The second map is also a collapsible extension by Cor. 7.5.

Remark 7.6. If $X$ in Prop. 7.2 is actually a $k$-groupoid for $k<\infty$, then the above proof can be refined to show that $X^{\Delta^{1}}$ is also an $k$-groupoid. We just need to verify that the cover

$$
\operatorname{Hom}\left(\Delta^{n} \times \Delta^{1}, X\right) \rightarrow \operatorname{Hom}\left(\Lambda_{j}^{n} \times \Delta^{1}, X\right)
$$

is an isomorphism for $n>k$. By Remark 3.8, this will be true provided we can show that the collapsible extension

$$
\Lambda_{j}^{n} \times \Delta^{1} \rightarrow \Delta^{n} \times \Delta^{1}
$$

is the pushout of maps of the form $\Lambda_{l}^{m} \rightarrow \Delta^{m}$ with $m>k$. Indeed, this is the case. The collapsible extension $\Lambda_{j}^{n} \rightarrow \Delta^{n}$ is the composition of two boundary extensions $\Lambda_{j}^{n} \rightarrow \partial \Delta^{n} \rightarrow \Delta^{n}$. We obtain $\partial \Delta^{n}$ from $\Lambda_{j}^{n}$ by attaching $\Delta^{n-1}$ along $\partial \Delta^{n-1} \rightarrow \Lambda_{j}^{n}$. Therefore, the collapsible extension obtained from $S=\Lambda_{j}^{n} \rightarrow \Delta^{n}=T$ from the auxiliary Lemma 7.4 above involves pushouts of maps of the form $\Lambda_{l}^{n} \rightarrow \Delta^{n}$ and $\Lambda_{l}^{n+1} \rightarrow \Delta^{n+1}$. (See the proof of Lemma 3.3.3 in [19].) From the proof of Lemma 7.3, we can then conclude that the collapsible extension (7.4) only involves pushouts along inclusions of horns which have dimension $>k$.

7.2. The iCFO structure. We arrive at our first main result:

Theorem 7.1. Let $(\mathrm{M}, \mathcal{T}, \mathcal{P})$ be a category equipped with a locally stalkwise pretopology with respect to a jointly conservative collection of points. The category $\operatorname{Gpd}_{\infty}[\mathrm{M}, \mathcal{T}]$, whose objects are $\infty$-groupoids in $(\mathrm{M}, \mathcal{T})$, and whose morphisms are simplicial maps is an incomplete category of fibrant objects in which:

- the weak equivalences are the stalkwise weak equivalences (Def. 5.1),

- the fibrations are the Kan fibrations (Def. 3.3),

- the acyclic fibrations are hypercovers (Def. 6.1).

In particular, the category of Lie $\infty$-groupoids

$$
\mathrm{Lie}_{\infty} \mathrm{Gpd}:=\mathrm{Gpd}_{\infty}\left[\mathrm{Mfd}, \mathcal{T}_{\text {subm }}, \mathcal{P}_{\text {Ban }}\right]
$$

is an incomplete category of fibrant objects in this way.

Let us make a number of important remarks before we proceed to the proof.

Remark 7.7.

(1) The acyclic fibrations are obviously determined by the weak equivalences and fibrations, i.e., those Kan fibrations that are also stalkwise weak equivalences. Since we are working with a locally stalkwise pretopology, it follows from Prop. 6.7 that acyclic fibrations are precisely the hypercovers. 
HOMOTOPY THEORY FOR LIE $\infty$-GROUPOIDS \& APPLICATION TO INTEGRATION 35

(2) In particular, if $M$ is small and has all finite limits, then $(M, \mathcal{T})$ is a "descent category" in the sense of Behrend-Getzler. (See proof of Cor. 7.16 for more details.) In this case, the iCFO structure on $\operatorname{Gpd}_{\infty}[\mathrm{M}, \mathcal{T}]$ agrees with the CFO structure of Thm. 3.6 in [3].

(3) In the proof of Thm 7.1, we use the assumption that $\mathcal{T}$ is a locally stalkwise pretopology only in the proof of Prop. 7.12, where we show that pullbacks of acyclic fibrations always exist.

(4) The fact that the category of Lie $\infty$-groupoids is an example of such an iCFO follows immediately from Prop. 6.12.

(5) The proof below of Thm. 7.1 can be enhanced to show that the category $\operatorname{Gpd}_{n}[\mathrm{M}, \mathcal{T}]$ of $n$-groupoids in $(\mathrm{M}, \mathcal{T})$ for $n<\infty$ also forms an iCFO. It follows from Remark 7.6 that the path object $X^{\Delta^{1}}$ associated to an $n$ groupoid $X$ will also be an $n$-groupoid. The only other modifications needed are in the proof of Prop. 7.10 below. (See Remark 7.11.)

The following collection of propositions proves Thm. 7.1 by directly verifying the axioms of Def. 2.1 We begin with the easiest axioms to verify:

Proposition 7.8 (Axioms 1, 2, 7).

(1) Every isomorphism in $\operatorname{Gpd}_{\infty}[\mathrm{M}, \mathcal{T}]$ is a stalkwise weak equivalence and a Kan fibration.

(2) If $f$ and $g$ are composable morphisms in $\mathrm{Gpd}_{\infty}[\mathrm{M}, \mathcal{T}]$, and any two of $f, g$, or $g \circ f$ are stalkwise weak equivalences, then so is the third.

(3) If $X$ is an $\infty$-groupoid in $(\mathrm{M}, \mathcal{T})$, then $X \rightarrow *$ is a Kan fibration.

Proof. (1) Obvious. (2) Follows from the fact that weak equivalences of simplicial sets satisfy the analogous 2 out of 3 axiom. (3) Follows immediately from Def. 3.4 .

This next proposition implies that the composition of two Kan fibrations is again a Kan fibration. A similar result appears in [17, Lemma 2.7] and also in [42, Theorem 2.17 (3)], where Kan fibrations are called " $n$-stacks".

Proposition 7.9 (Axiom 3). Let $f: X \rightarrow Y$ and $g: Y \rightarrow Z$ be morphisms of simplicial objects in $\mathrm{M}$. If $f$ and $g$ satisfy $\operatorname{Kan}(n, j)$ and the sheaf

$$
\operatorname{Hom}\left(\Lambda_{j}^{n} \stackrel{\iota}{\rightarrow} \Delta^{n}, X \stackrel{g \circ f}{\longrightarrow} Z\right)
$$

is representable, then $g \circ f$ also satisfies $\operatorname{Kan}(n, j)$. Similarly, if $f$ and $g$ satisfy Kan! $(n, j)$, then $g \circ f$ also satisfies Kan! $(n, j)$.

Proof. The proposition, in fact, follows from Wolfson's Theorem 2.17 (3) in [42]. Indeed, axiom 1 in our Def. 6.5 implies axiom 3 in [42, Sec. 2] for a category equipped with a subcategory of covers. For the reader's convenience we recall the proof from [42] using our notation. 
We have the following diagram containing three pullback squares:

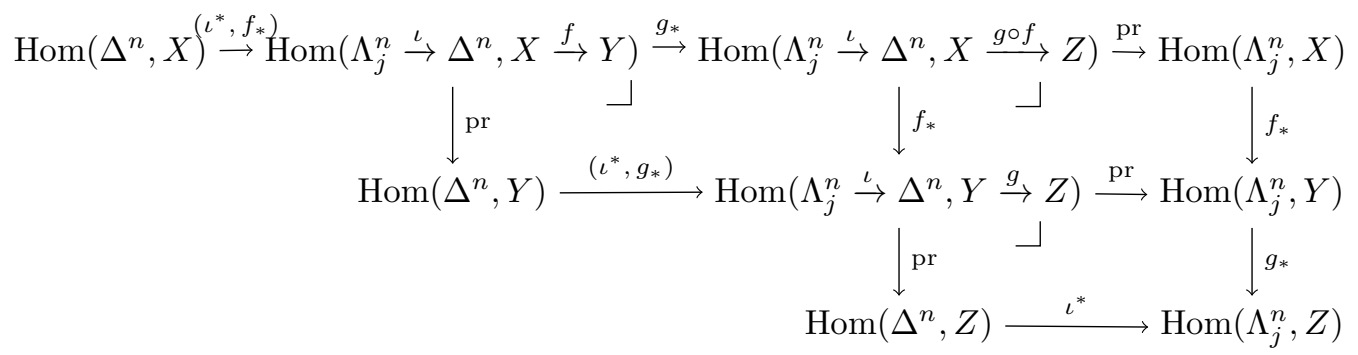

If $g$ satisfies $\operatorname{Kan}(n, j)$, then $\operatorname{Hom}\left(\Delta^{n}, Y\right) \stackrel{\left(\iota^{*}, g_{*}\right)}{\rightarrow} \operatorname{Hom}\left(\Lambda_{j}^{n} \stackrel{\iota}{\rightarrow} \Delta^{n}, Y \stackrel{g}{\rightarrow} Z\right)$ is a cover. Hence, $\operatorname{Hom}\left(\Lambda_{j}^{n} \stackrel{\iota}{\rightarrow} \Delta^{n}, X \stackrel{f}{\rightarrow} Y\right) \stackrel{g_{*}}{\rightarrow} \operatorname{Hom}\left(\Lambda_{j}^{n} \stackrel{\iota}{\rightarrow} \Delta^{n}, X \stackrel{g \circ f}{\longrightarrow} Z\right)$ is a cover. If $f$ satisfies $\operatorname{Kan}(n, j)$, then $\operatorname{Hom}\left(\Delta^{n}, X\right) \stackrel{\left(\iota^{*}, f_{*}\right)}{\longrightarrow} \operatorname{Hom}\left(\Lambda_{j}^{n} \stackrel{\iota}{\rightarrow} \Delta^{n}, X \stackrel{f}{\rightarrow} Y\right)$ is a cover. Hence, the composition

$$
g_{*} \circ\left(\iota^{*}, f_{*}\right)=\left(\iota^{*},(g \circ f)_{*}\right): \operatorname{Hom}\left(\Delta^{n}, X\right) \rightarrow \operatorname{Hom}\left(\Lambda_{j}^{n} \stackrel{\iota}{\rightarrow} \Delta^{n}, X \stackrel{g \circ f}{\longrightarrow} Z\right)
$$

is a cover, and so $g \circ f$ satisfies $\operatorname{Kan}(n, j)$. The same argument mutatis mutandis shows that if $f$ and $g$ satisfy $\operatorname{Kan!}(n, j)$, then so does $g \circ f$.

We next show that the pullback of a Kan fibration is a Kan fibration, provided the pullback exists in $\mathrm{Gpd}_{\infty}[\mathrm{M}, \mathcal{T}]$. As previously mentioned, this is the only difference between the axioms for an iCFO and Brown's axioms for a CFO: we do not require the pullback along a fibration to exist in general. It turns out, for $\infty$-groupoids, this generalization is in fact quite mild. The pullback of along a Kan fibration will always exist provided the corresponding pullback of the 0 -simplices exists in $\mathrm{M}$. (The same result appears as Thm. 2.17 (4) in [42].)

Proposition 7.10 (Axiom 4). Let $f: X \rightarrow Y$ be a Kan fibration in $\mathrm{Gpd}_{\infty}[\mathrm{M}, \mathcal{T}]$ and $g: Z \rightarrow Y$ a morphism in $\mathrm{Gpd}_{\infty}[\mathrm{M}, \mathcal{T}]$. If the pullback $Z_{0} \times_{Y_{0}} X_{0}$ exists in $\mathrm{M}$, then:

(1) the pullback $Z_{n} \times_{Y_{n}} X_{n}$ exists in $\mathrm{M}$ for all $n \geq 0$,

(2) the morphism $Z \times_{Y} X \stackrel{p_{f}}{\longrightarrow} Z$ induced by pulling back $f$ along $g$ is a Kan fibration between simplicial objects in $\mathrm{M}$,

(3) the pullback $Z \times_{Y} X$ is an object of $\mathrm{Gpd}_{\infty}[\mathrm{M}, \mathcal{T}]$.

Proof. For convenience, we use the following notation below: If $K$ is a simplicial set and $W$ is a simplicial object in $\mathrm{M}$, then $K(W)$ is the sheaf $\operatorname{Hom}(K, W)$. Also, we denote by $\operatorname{Hom}\left(\iota, p_{f}\right)$ the sheaf $\operatorname{Hom}\left(\Lambda_{j}^{n} \stackrel{\iota}{\rightarrow} \Delta^{n}, Z \times_{Y} X \stackrel{p_{f}}{\rightarrow} Z\right)$. Finally, we do not distinguish between a simplicial object $W$ in $\mathrm{M}$, and the representable sheaf $\mathbf{y} W$.

We shall prove statements (1) and (2) simultaneously: For all $n \geq 0$, and $0 \leq$ $j \leq n$, we wish to show that morphism of sheaves

$$
Z_{n} \times_{Y_{n}} X_{n} \stackrel{\left(\iota^{*}, p_{f *}\right)}{\longrightarrow} \operatorname{Hom}\left(\iota, p_{f}\right)
$$

is represented by a cover in M. It follows from the definition Def. 3.1, that for a fixed simplicial set $K$, the functor $\operatorname{Hom}(K,-): s \mathrm{M} \rightarrow \mathrm{Sh}(\mathrm{M})$ preserves limits. 
Therefore, we have an isomorphism of sheaves

$$
\begin{aligned}
\operatorname{Hom}\left(\iota, p_{f}\right) & :=\Lambda_{j}^{n}\left(Z \times_{Y} X\right) \times_{\Lambda_{j}^{n}(Z)} Z_{n} \\
& \cong\left(\Lambda_{j}^{n}(Z) \times_{\Lambda_{j}^{n}(Y)} \Lambda_{j}^{n}(X)\right) \times_{\Lambda_{j}^{n}(Z)} Z_{n},
\end{aligned}
$$

and a commuting diagram of pullback squares

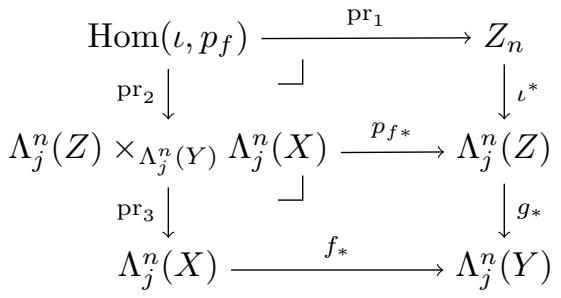

Hence, the pasting law for pullbacks gives an isomorphism of sheaves

$$
\operatorname{Hom}\left(\iota, p_{f}\right) \cong \Lambda_{j}^{n}(X) \times_{\Lambda_{j}^{n}(Y)} Z_{n} .
$$

The above isomorphism gives another commuting diagram of pullback squares, which via the universal property, contains the morphism (7.5):

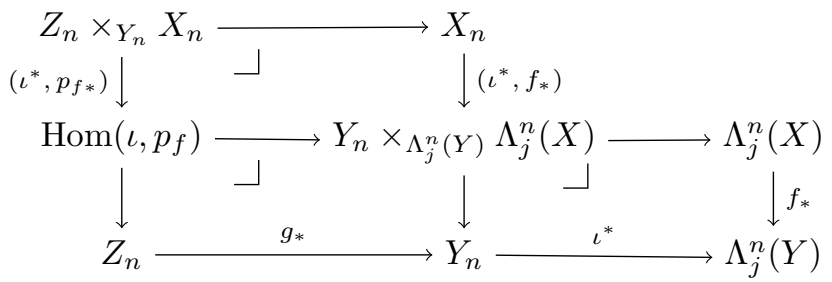

Note that since $f: X \rightarrow Y$ is a Kan fibration, the morphism $X_{n} \stackrel{\left(\iota^{*}, f_{*}\right)}{\longrightarrow} Y_{n} \times_{\Lambda_{j}^{n}(Y)}$ $\Lambda_{j}^{n}(X)$ is represented by a cover. Hence, to show that the morphism (7.5) is represented by a cover, the above diagram implies that it is sufficient to show that the sheaf

$$
\Lambda_{j}^{n}(X) \times_{\Lambda_{j}^{n}(Y)} Z_{n}
$$

is representable for all $n \geq 1$ and $0 \leq j \leq n$.

First consider the $n=1$ case. Diagram (7.6) and the isomorphism (7.7) imply that we have the pullback diagram

$$
\begin{gathered}
X_{0} \times_{Y_{0}} Z_{1} \rightarrow Z_{1} \\
\downarrow-\downarrow^{*}=d_{j} \\
Z_{0} \times_{Y_{0}} X_{0} \rightarrow Z_{0}
\end{gathered}
$$

The sheaf $Z_{0} \times_{Y_{0}} X_{0}$ is representable by hypothesis, and since $Z$ is an $\infty$-groupoid, $Z_{1} \stackrel{d_{j}}{\rightarrow} Z_{0}$ is a cover. Therefore, for $n=1$ and $j=0,1$, the sheaf (7.9) is representable, $Z_{1} \times_{Y_{1}} X_{1}$ is representable, and $p_{f}: Z \times_{Y} X \rightarrow Z$ satisfies $\operatorname{Kan}(1, j)$.

Now suppose $p_{f}$ satisfies $\operatorname{Kan}(m, j)$ for all $1 \leq m<n$ and $0 \leq j \leq m$. This plus the fact that $\Lambda_{j}^{n}$ is a collapsible subset of $\Delta^{n}$, allows us to apply Lemma 3.9 to conclude that $\operatorname{Hom}\left(\iota_{n, j}, p_{f}\right) \cong \Lambda_{j}^{n}(X) \times_{\Lambda_{j}^{n}(Y)} Z_{n}$. is representable. Hence, $p_{f}: Z \times_{Y} X \rightarrow Z$ satisfies $\operatorname{Kan}(n, j)$, and so it is a Kan fibration. 
Statement (3) immediately follows. Indeed, since $Z$ is an $\infty$-groupoid, $Z \rightarrow *$ is a Kan fibration. Therefore, Prop. 7.9 implies that $Z \times_{Y} X \rightarrow *$ is also a Kan fibration.

Remark 7.11. If $X, Y$, and $Z$ in the statement of Prop. 7.10 are $k$-groupoids, $k<\infty$, then one can show that the pullback $Z \times_{Y} X$, if it exists, is also a $k$ groupoid. As previously mentioned in Remark 7.7, this fact helps show that the category $\mathrm{Gpd}_{k}[\mathrm{M}, \mathcal{T}]$ forms an iCFO. We just need to verify that the morphism

$$
Z_{n} \times_{Y_{n}} X_{n} \rightarrow \Lambda_{j}^{n}(Z) \times_{\Lambda_{j}^{n}(Y)} \Lambda_{j}^{n}(X)
$$

is an isomorphism for $n>k$. Since $Z$ is a $k$-groupoid, the pullback diagram (7.6) implies that

$$
\operatorname{Hom}\left(\iota_{n, j}, p_{f}\right) \stackrel{\mathrm{pr}_{2}}{\longrightarrow} \Lambda_{j}^{n}(Z) \times_{\Lambda_{j}^{n}(Y)} \Lambda_{j}^{n}(X)
$$

is an isomorphism. Since $f: X \rightarrow Y$ is a Kan fibration between $k$-groupoids, it is not hard to show that the morphism

$$
X_{n} \stackrel{\left(\iota^{*}, f_{*}\right)}{\longrightarrow} Y_{n} \times_{\Lambda_{j}^{n}(Y)} \Lambda_{j}^{n}(X)
$$

is an isomorphism for all $n>k$. Hence, the pullback diagram (7.8) implies that

$$
Z_{n} \times_{Y_{n}} X_{n} \stackrel{\left(\iota^{*}, p_{f *}\right)}{\longrightarrow} \operatorname{Hom}\left(\iota_{n, j}, p_{f}\right)
$$

is an isomorphism. Composing this with the isomorphism (7.12), we conclude that (7.11) is an isomorphism.

Proposition 7.10 also makes it easy to show that the pullbacks of acyclic fibrations always exist in $\mathrm{Gpd}_{\infty}[\mathrm{M}, \mathcal{T}]$, and are always acyclic fibrations. (Since acyclic fibrations turn out to be equivalent to hypercovers, this result is equivalent to [43, Lemma 2.8].)

Proposition 7.12 (Axiom 5). Let $f: X \rightarrow Y$ be an acyclic fibration in $\mathrm{Gpd}_{\infty}[\mathrm{M}, \mathcal{T}]$ and $g: Z \rightarrow Y$ a morphism in $\mathrm{Gpd}_{\infty}[\mathrm{M}, \mathcal{T}]$. Then the morphism $Z \times_{Y} X \stackrel{q_{f}}{\longrightarrow} Z$ induced by pulling back $f$ along $g$ is an acyclic fibration.

Proof. Since $f: X \rightarrow Y$ is an acyclic fibration, Prop. 6.7 implies that $f$ is a hypercover. Then, by definition, $f_{0}: X_{0} \rightarrow Y_{0}$ is a cover, and hence the pullback $Z_{0} \times_{Y_{0}} X_{0}$ exists in M. Proposition 7.10 therefore implies that the morphism $Z \times_{Y} X \stackrel{q_{f}}{\longrightarrow} Z$ is a Kan fibration in $\operatorname{Gpd}_{\infty}[\mathrm{M}, \mathcal{T}]$.

Let $\mathrm{p}$ be a point. Then $\mathrm{p} f: \mathrm{p} X \rightarrow \mathrm{p} Y$ is an acyclic fibration of simplicial sets. By definition, points preserve finite limits. Hence, $\mathrm{p}\left(Z \times_{Y} X\right) \stackrel{\mathrm{p} q_{f}}{\longrightarrow} \mathrm{p} Z$ is the pullback of $\mathrm{p} f$, and is therefore a weak equivalence of simplicial sets. So we conclude $q_{f}$ is a stalkwise weak equivalence, hence an acyclic fibration.

Finally, we show that if $X \in \mathrm{Gpd}_{\infty}[\mathrm{M}, \mathcal{T}]$, then the $\infty$-groupoid $X^{\Delta^{1}}$ constructed in Prop. 7.2 is a path object for $X$. Let

$$
X^{\Delta^{1}} \stackrel{\left(d^{0 *}, d^{1 *}\right)}{\longrightarrow} X \times X \cong X^{\Delta^{0} \cup \Delta^{0}},
$$

and

$$
s^{0 *}: X \rightarrow X^{\Delta^{1}}
$$

denote the morphisms (7.2) and (7.3), respectively, induced by the inclusions $\partial \Delta^{1} \cong$ $\Delta^{0} \cup \Delta^{0} \stackrel{d^{0} \cup d^{1}}{\longrightarrow} \Delta^{1}$ and the constant map $s^{0}: \Delta^{1} \rightarrow \Delta^{0}$, respectively. 
Proposition 7.13 (Axiom 6). Let $X \in \mathrm{Gpd}_{\infty}[\mathrm{M}, \mathcal{T}]$. Then

$$
X \stackrel{s^{0 *}}{\longrightarrow} X^{\Delta^{1}} \stackrel{\left(d^{0 *}, d^{1 *}\right)}{\longrightarrow} X \times X .
$$

is a factorization of the diagonal map $X \times X$ into a stalkwise weak equivalence $s_{0}^{*}: X \rightarrow X^{\Delta^{1}}$ followed by a Kan fibration $X^{\Delta^{1}} \stackrel{\left(d^{0 *}, d^{1 *}\right)}{\longrightarrow} X \times X$.

To prove the above, we'll need the following lemma, whose proof we postpone to Appendix A.

Lemma 7.14. The inclusion of simplicial sets

$$
\Lambda_{j}^{n} \times \Delta^{1} \sqcup_{\Lambda_{j}^{n} \times \partial \Delta^{1}} \Delta^{n} \times \partial \Delta^{1} \rightarrow \Delta^{n} \times \Delta^{1}
$$

is a collapsible extension.

Proof of Prop. 7.12. First, observe that $d^{i *} \circ s^{0 *}=\mathrm{id}_{X}$. This follows from fact that the assignment $K \mapsto(\mathbf{y} X)^{K}$, where $(\mathbf{y} X)^{K}$ is the simplicial sheaf (7.1), is a contravariant functor from simplicial sets to simplicial sheaves. Hence, (7.13) is a factorization of the diagonal map.

Next, since we have an isomorphism of sheaves $\mathbf{y}\left(X^{\Delta^{1}}\right) \cong(\mathbf{y} X)^{\Delta^{1}}$, Prop. 7.1 implies that $s_{0}^{*}: X \rightarrow X^{\Delta^{1}}$ is a stalkwise weak equivalence.

Now we show $f:=\left(d^{0 *}, d^{1 *}\right): X^{\Delta^{1}} \rightarrow X \times X$ satisfies the condition $\operatorname{Kan}(n, j)$ for all $n \geq 1$ and $0 \leq j \leq n$, i.e. the morphism of sheaves

$$
X_{n}^{\Delta^{1}} \stackrel{\left(\iota_{n, j}^{*}, f_{*}\right)}{\longrightarrow} \operatorname{Hom}\left(\iota_{n, j}, f\right),
$$

where $\operatorname{Hom}\left(\iota_{n, j}, f\right):=\operatorname{Hom}\left(\Lambda_{j}^{n} \stackrel{\iota_{n, j}}{\longrightarrow} \Delta^{n}, X^{\Delta^{1}} \stackrel{f}{\rightarrow} X \times X\right)$ is represented by a cover. It follows from Prop. 4.5 that for any finitely generated simplicial sets $K$ and $L$, we have an isomorphism of sheaves

$$
\operatorname{Hom}\left(L, X^{K}\right) \cong \operatorname{Hom}(L \times K, X) .
$$

Therefore, we have the following isomorphisms of sheaves

$$
\begin{aligned}
\operatorname{Hom}\left(\iota_{n, j}, f\right) & \cong \operatorname{Hom}\left(\Lambda_{j}^{n} \times \partial \Delta^{1}, X\right) \times_{\operatorname{Hom}\left(\Lambda_{j}^{n} \times \partial \Delta^{1}, X\right)} \operatorname{Hom}\left(\Lambda_{j}^{n} \times \Delta^{1}, X\right) \\
& \cong \operatorname{Hom}\left(\Lambda_{j}^{n} \times \Delta^{1} \sqcup_{\Lambda_{j}^{n} \times \partial \Delta^{1}} \Delta^{n} \times \partial \Delta^{1}, X\right)
\end{aligned}
$$

Hence, showing that $f$ satisfies $\operatorname{Kan}(n, j)$ is equivalent to showing that the morphism of sheaves

$$
\operatorname{Hom}\left(\Delta^{n} \times \Delta^{1}, X\right) \stackrel{\left(\iota_{n, j}^{*}, f_{*}\right)}{\longrightarrow} \operatorname{Hom}\left(\Lambda_{j}^{n} \times \Delta^{1} \sqcup_{\Lambda_{j}^{n} \times \partial \Delta^{1}} \Delta^{n} \times \partial \Delta^{1}, X\right)
$$

is a cover. Lemma 7.14 implies that the inclusion $\Lambda_{j}^{n} \times \Delta^{1} \sqcup_{\Lambda_{j}^{n} \times \partial \Delta^{1}} \Delta^{n} \times \partial \Delta^{1} \rightarrow$ $\Delta^{n} \times \Delta^{1}$ is a collapsible extension. Hence, Lemma 3.7 implies that in order to show (7.14) is a cover, it suffices to show that

$$
\operatorname{Hom}\left(\iota_{n, j}, f\right) \cong \operatorname{Hom}\left(\Lambda_{j}^{n} \times \Delta^{1} \sqcup_{\Lambda_{j}^{n} \times \partial \Delta^{1}} \Delta^{n} \times \partial \Delta^{1}, X\right)
$$

is representable for all $n$ and $j$.

Consider the $n=1$ case. Then we have the pullback square

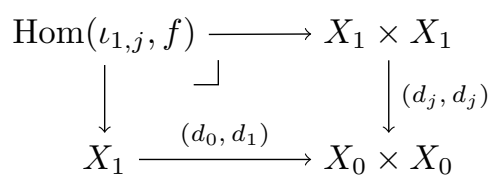


Since $X \times X$ is an $\infty$-groupoid, the map $X_{1} \times X_{1} \stackrel{d_{j}, d_{j}}{\longrightarrow} X_{0} \times X_{0}$ is a cover. Hence, the pullback $\operatorname{Hom}\left(\iota_{1, j}, f\right)$ exists and so $f$ satisfies $\operatorname{Kan}(1, j)$. Now if $n>1$ and $f$ satisfies $\operatorname{Kan}(m, j)$ for all $m<n$ and $1 \leq j \leq m$, then Lemma 3.9 implies that $\operatorname{Hom}\left(\iota_{n, j}, f\right)$ is representable.

7.2.1. Simplicial localization for $\operatorname{Gpd}_{\infty}[\mathrm{M}, \mathcal{T}]$. We note that the path object $X^{\Delta^{1}}$ used in the proof of Thm. 7.1 is functorial, in the sense of Sec. 2.2. This can be easily deduced from the fact that $X^{\Delta^{1}}$ represents the sheaf $(\mathbf{y} X)^{\Delta^{1}}$ (Prop. 7.2). The iCFO structure on $\mathrm{Lie}_{\infty}$ Gpd in particular is equipped with both functorial path objects, as well as functorial pullbacks of acyclic fibrations. Indeed, the pullbacks in this case are characterized in each simplicial dimension by the unique Banach manifold structure on the set-theoretic fiber product. (See, for example, Prop. 2.5 and Prop. 2.6 of [25].) Hence, for a small full subcategory of Lie $n$-groupoids closed under the iCFO structure, Thm. 2.13 would provide a convenient description of its simplicial localization. A potentially useful example of this sort, which will be studied in future work, is the category of $n$-groupoids internal to the category of separable Banach manifolds ${ }^{1}$.

7.3. Alternative characterization of weak equivalences. The incorporation of stalkwise weak equivalences into our iCFO structure for $\operatorname{Gpd}_{\infty}[\mathrm{M}, \mathcal{T}]$ turns out to be quite convenient for some applications. However, in general, verifying directly that a morphism is a stalkwise weak equivalence could be cumbersome. Furthermore, we have the aesthetically inelegant fact that the stalkwise weak equivalences are the only piece of the iCFO structure on $\operatorname{Gpd}_{\infty}[\mathrm{M}, \mathcal{T}]$ which requires us to leave the realm of simplicial objects in $\mathrm{M}$ for the larger world of simplicial sheaves on $\mathrm{M}$.

Fortunately, as was mentioned in Sec. 2, the weak equivalences in an iCFO are completely determined by the acyclic fibrations. This very useful fact is emphasized in the work of Behrend and Getzler [3] on CFOs for higher geometric groupoids in descent categories. What this implies in particular for the $\mathrm{iCFO}$ structure on $\operatorname{Gpd}_{\infty}[\mathrm{M}, \mathcal{T}]$, is the following: If $f: X \rightarrow Y$ is a morphism in $\operatorname{Gpd}_{\infty}[\mathrm{M}, \mathcal{T}]$, we consider the pullback diagram

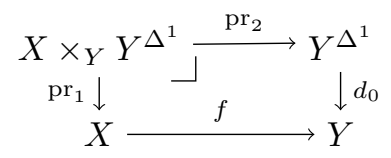

Then it follows from Lemma 2.4 and Prop. 2.5 (and Thm. 7.1) that $f: X \rightarrow Y$ is a stalkwise weak equivalence if and only if the composition

$$
p_{f}: X \times_{Y} Y^{\Delta^{1}} \stackrel{\mathrm{pr}_{2}}{\longrightarrow} Y^{\Delta^{1}} \stackrel{d_{1}}{\longrightarrow} Y
$$

is a hypercover. Moreover, the path object construction can be avoided altogether, and weak equivalences can be characterized directly in terms of $f$ and covers between representable sheaves.

To give just a simple example, denote by $\iota_{a}: \Delta^{n} \rightarrow \Delta^{1} \times \Delta^{n}$ for $a=0,1$ the inclusions $m \mapsto(a, m)$. Similarly, there are the inclusions $\partial \iota_{a}: \partial \Delta^{n} \rightarrow \Delta^{1} \times \partial \Delta^{n}$.

\footnotetext{
${ }^{1}$ We thank E. Getzler for this observation.
} 
There is the pushout diagram

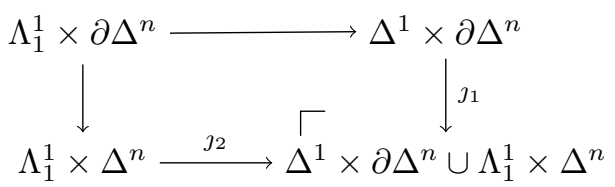

The following is parallel to the first step in the proof of Thm. 5.1 in [3]:

Proposition 7.15. A morphism $f: X \rightarrow Y$ in $\operatorname{Gpd}_{\infty}[\mathrm{M}, \mathcal{T}]$ is a stalkwise weak equivalence if and only if, for all $n \geq 0$ the morphism in $\mathrm{M}$

$\operatorname{Hom}\left(\Delta^{n} \stackrel{i_{1}}{\rightarrow} \Delta^{1} \times \Delta^{n}, X \stackrel{f}{\rightarrow} Y\right) \rightarrow \operatorname{Hom}\left(\partial \Delta^{n} \stackrel{\jmath_{1} \circ \partial \iota_{1}}{\longrightarrow} \Delta^{1} \times \partial \Delta^{n} \cup \Lambda_{1}^{1} \times \Delta^{n}, X \stackrel{f}{\rightarrow} Y\right)$

is a cover.

Proof. First, it follows from Lemma 2.4 in [43] that the sheaf

$$
\operatorname{Hom}\left(j, p_{f}\right):=\operatorname{Hom}\left(\partial \Delta^{n} \stackrel{j}{\rightarrow} \Delta^{n}, X \times_{Y} Y^{\Delta^{1}} \stackrel{p_{f}}{\longrightarrow} Y\right)
$$

is representable. From the discussion preceding the proposition, we know $f: X \rightarrow Y$ is a weak equivalence if and only if for all $n \geq 0$, the morphism in $\mathrm{M}$

$$
X_{n} \times_{Y_{n}} Y_{n}^{\Delta^{1}} \rightarrow \operatorname{Hom}\left(j, p_{f}\right)
$$

is a cover. Since $Y_{n}^{\Delta^{1}} \cong \operatorname{Hom}\left(\Delta^{1} \times \Delta^{n}, Y\right)$, there is the pullback square

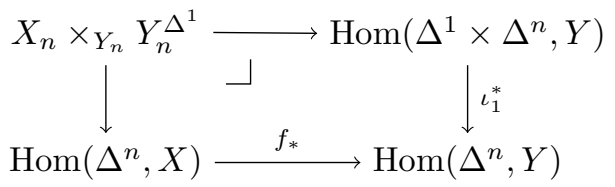

Hence,

$$
X_{n} \times_{Y_{n}} Y_{n}^{\Delta^{1}} \cong \operatorname{Hom}\left(\Delta^{n} \stackrel{i_{1}}{\rightarrow} \Delta^{1} \times \Delta^{n}, X \stackrel{f}{\rightarrow} Y\right)
$$

To complete the proof, we just need to show

$$
\operatorname{Hom}\left(j, p_{f}\right) \cong \operatorname{Hom}\left(\partial \Delta^{n} \stackrel{\jmath_{1} \circ \partial \iota_{1}}{\longrightarrow} \Delta^{1} \times \partial \Delta^{n} \cup \Lambda_{1}^{1} \times \Delta^{n}, X \stackrel{f}{\rightarrow} Y\right) .
$$

This follows from pasting together the following pullback squares:

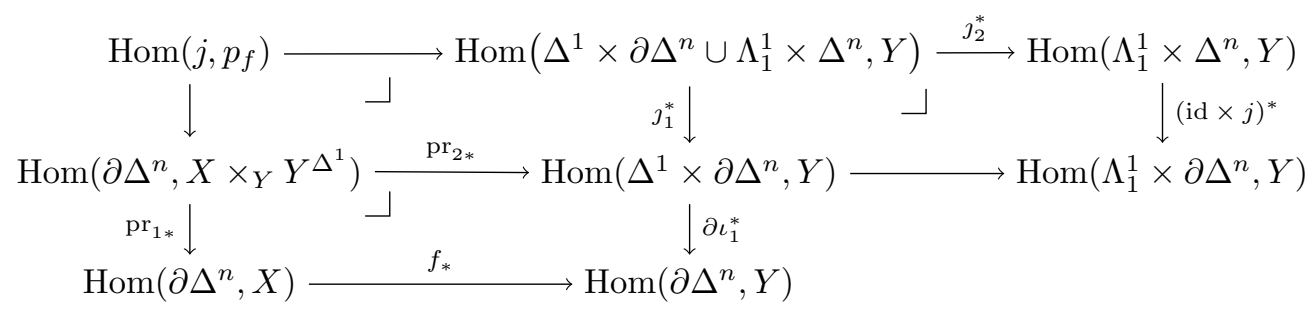

Theorem 5.1 in [3] further shows that if the category of $n$-groupoids in $(\mathrm{M}, \mathcal{T})$ form a category of fibrant objects, then $f: X \rightarrow Y$ is a weak equivalence if and only if the morphism

$$
\operatorname{Hom}\left(\Delta^{n} \rightarrow \Delta^{n+1}, X \rightarrow Y\right) \rightarrow \operatorname{Hom}\left(\partial \Delta^{n} \rightarrow \Lambda_{n+1}^{n+1}, X \rightarrow Y\right)
$$


is a cover for $n \geq 0$. This turns out to be true in our iCFO case as well.

Corollary 7.16. A morphism $f: X \rightarrow Y$ in Lie $_{\infty}$ Gpd is a stalkwise weak equivalence if and only if the natural morphism (7.16) is a cover for $n \geq 0$.

Proof. By Proposition 7.15, we see that $f: X \rightarrow Y$ in $\mathrm{Lie}_{\infty} \mathrm{Gpd}$ is a stalkwise weak equivalence if and only if the morphism (7.15) is a cover for all $n \geq 0$. The morphisms (7.15) and (7.16) are exactly the morphisms (5.2) and (5.1), respectively, in [3]. The sources and targets for the morphisms in [3] are $n$-groupoids in a descent category of spaces: a small category with finite limits, equipped with a subcategory of covers closed under pullback, which satisfy a " 2 of 3" property. (Axioms D1, D2, and D3, respectively in [3].) A category equipped with a locally stalkwise pretopology satisfies all of these axioms, except D1. Indeed, D2 is included in the definition of a pretopology, and D3 follows from Def. 6.5. Even though D1 is not satisfied in this context, the proof of Thm. 5.1 in [3] still applies. A direct verification shows that all limits appearing in the proof exist in $(\mathrm{M}, \mathcal{T})$. And clearly, the proof works for $n=\infty$.

Remark 7.17. We also mention that a characterization of weak equivalences similar to (7.16) between Lie 2-groupoids can be deduced using properties of the join construction of simplicial sets and the theory of Morita bibundles developed in Li's Ph.D. thesis [26]. This fact is generalized to all Lie $n$-groupoids in [4], which provides another interpretation of the combinatorial formula (7.16).

\section{LIE $n$-ALGEBRAS}

In this section, we summarize the main results we will need from the companion paper [36] concerning the homotopy theory of finite type Lie $n$-algebras, and we refer the reader there for complete proofs and details. Throughout this section and the remainder of this paper, we adopt the notation and conventions from [36, Sec. 2 ; Sec. 3].

8.1. $L_{\infty}$-algebras and their morphisms. We begin with a quick review of basic facts and definitions concerning $L_{\infty}$-algebras and their morphisms. We follow the presentation in [36, Sec. 3] which is based on the standard reference [24].

Recall that an $L_{\infty}$-algebra $(L, \ell)$ is a $\mathbb{Z}$-graded $\mathbb{R}$-vector space $L$ equipped with a collection $\ell=\left\{\ell_{1}, \ell_{2}, \ell_{3}, \ldots\right\}$ of graded skew-symmetric linear maps (or brackets)

$$
\ell_{k}: \Lambda^{k} L \rightarrow L, \quad 1 \leq k<\infty
$$

with $\left|\ell_{k}\right|=k-2$, satisfying an infinite sequence of Jacobi-like identities of the form:

$$
\sum_{\substack{i+j=m+1, \sigma \in \operatorname{Sh}(i, m-i)}}(-1)^{\sigma} \epsilon(\sigma)(-1)^{i(j-1)} l_{j}\left(l_{i}\left(x_{\sigma(1)}, \ldots, x_{\sigma(i)}\right), x_{\sigma(i+1)}, \ldots, x_{\sigma(m)}\right)=0
$$

for all $m \geq 1$. Above, the permutation $\sigma$ ranges over all $(i, m-i)$ unshuffles, and $\epsilon(\sigma)$ denotes the Koszul sign. In particular, Eq. 8.1 implies that $\left(L, \ell_{1}\right)$ is a (homological) chain complex.

Equivalently, a $L_{\infty}$-structure on a graded vector space $L$ is a degree -1 codifferential $\delta$ on the reduced cocommutative coalgebra $\bar{S}(\mathbf{s} L)=\bigoplus_{i \geq 1} S^{i}(\mathbf{s} L)$. See [36, Sec. 2.4; Sec. 3.1] for further details. Here $\mathbf{s} L$ denotes the suspension of the graded vector space $L$, i.e., $\mathbf{s} L_{i}:=L[-1]_{i}=L_{i-1}$. 
A (weak) $L_{\infty}$-morphism $f:(L, \ell) \rightarrow\left(L^{\prime}, \ell^{\prime}\right)$ is a collection $f=\left\{f_{1}, f_{2}, \ldots\right\}$ of graded skew-symmetric linear maps $f_{k}: \Lambda^{k} L \rightarrow L^{\prime} \quad 1 \leq k<\infty$ with $\operatorname{deg} f_{k}=k-1$, satisfying an infinite sequence of equations of the form:

$$
\begin{aligned}
& \sum_{j+k=m+1} \sum_{\sigma} \pm f_{j}\left(l_{k}\left(x_{\sigma}(1), \ldots, x_{\sigma(k)}\right), x_{\sigma(k+1)}, \ldots x_{\sigma(m)}\right) \\
& +\sum_{\substack{1 \leq t \leq m \\
i_{1}+\cdots i_{t}=m}} \sum_{\tau} \pm l_{t}^{\prime}\left(f_{i_{1}}\left(x_{\tau(1)}, \ldots, x_{\tau\left(i_{1}\right)}\right), f_{i_{2}}\left(x_{\tau\left(i_{1}+1\right)}, \ldots, x_{\tau\left(i_{1}+i_{2}\right)}\right),\right. \\
& \left.\quad \ldots, f_{i_{t}}\left(x_{\tau\left(i_{1}+\cdots+i_{t-1}+1\right)}, \ldots, x_{\tau(m)}\right)\right)=0 .
\end{aligned}
$$

Above $\sigma$ ranges over all $(k, m-k)$ unshuffles, and $\tau$ ranges through certain $\left(i_{1}, \ldots, i_{t}\right)$ unshuffles. (See, for example, [1, Def. 2.3].) More conveniently, a morphism between $L_{\infty}$-algebras $L$ and $L^{\prime}$ is equivalently a degree 0 morphism of dg coalgebras $F:(\bar{S}(\mathbf{s} L), \delta) \rightarrow\left(\bar{S}\left(\mathbf{s} L^{\prime}\right), \delta^{\prime}\right)$. See again [36, Sec. 2.4; Sec. 3.1] for further details. In particular, treating $L_{\infty}$ morphisms as dg coalgebra morphisms gives us a clear way to compose them [36, Eq. 2.6]. It is typical to consider the category $\mathrm{L}_{\infty} \mathrm{Alg}$ of $L_{\infty}$-algebras and $L_{\infty}$-morphisms as a full subcategory of the category of dg cocommutative coalgebras.

\section{Remark 8.1.}

- As in [36], we will write morphisms in $\mathrm{L}_{\infty}$ Alg using a single lower-case letter, e.g.

$$
f:(L, \ell) \rightarrow\left(L^{\prime}, \ell^{\prime}\right),
$$

and the $k$-ary map in the collection $f$ will always be denoted by $f_{k}$.

- Recall that if $f:(L, \ell) \rightarrow\left(L^{\prime}, \ell^{\prime}\right)$ is a $L_{\infty}$-morphism, then

$$
H\left(f_{1}\right):\left(H_{0}(L),[\cdot, \cdot]\right) \rightarrow\left(H_{0}\left(L^{\prime}\right),[\cdot, \cdot]^{\prime}\right)
$$

is a morphism of Lie algebras, where the above Lie brackets are induced by the bilinear brackets $\ell_{2}$ and $\ell_{2}^{\prime}$, respectively.

Next we recall several important classes of $L_{\infty}$-morphisms:

Definition 8.2. Let $f:(L, \ell) \rightarrow\left(L^{\prime}, \ell^{\prime}\right)$ be a morphism of $L_{\infty}$-algebras.

(1) We say $f$ is a $L_{\infty}$-isomorphism iff the linear map $f_{1}: L \rightarrow L^{\prime}$ is an isomorphism of graded vector spaces.

(2) We say $f$ is a $L_{\infty}$-quasi-isomorphism iff the chain map $f_{1}$ is a quasiisomorphism, i.e. the induced map on homology $H\left(f_{1}\right): H(L) \rightarrow H\left(L^{\prime}\right)$ is an isomorphism of graded vector spaces.

(3) We say $f$ is a strict $L_{\infty}$-morphism iff $f_{k}=0$ for all $k \geq 2$. In this case we write $f=f_{1}:(L, \ell) \rightarrow\left(L^{\prime}, \ell\right)$ and it follows that every $k$-ary bracket $\ell_{k}$ is preserved by the chain map $f_{1}$ :

$$
\ell_{k}^{\prime} \circ f_{1}^{\otimes k}=f_{1} \circ \ell_{k} \quad \text { for all } k \geq 1 .
$$

8.2. Finite type Lie $n$-algebras. Let $n \in \mathbb{N} \cup\{\infty\}$. We recall that a $L_{\infty}$-algebra $\left(L, \ell_{k}\right)$ is a Lie $n$-algebra iff the graded vector space $L$ is concentrated in the first $n-1$ non-negative degrees, i.e. $L=\bigoplus_{i>0}^{n-1} L_{i}$. The standard reference for Lie $n$-algebras is [6, Def. 4.3.2]. See [36, Sec. 3.2] for a list of relevant examples. For a fixed $n \in \mathbb{N} \cup\{\infty\}$, we denote by $\mathrm{Lie}_{n} \mathrm{Alg}$ the full subcategory of $\mathrm{L}_{\infty} \mathrm{Alg}$ whose objects are Lie $n$-algebras. 
If $(L, \ell)$ is a Lie $n$-algebra and each $L_{i}$ is a finite-dimensional vector space, then we say $(L, \ell)$ is a finite type Lie $n$-algebra, and we denote by $\operatorname{Lie}_{n} A l g$ fin the category whose objects are finite type Lie $n$-algebras, and whose morphisms are (weak) $L_{\infty}$-morphisms.

8.2.1. The Chevalley-Eilenberg algebra. Throughout the companion paper [36], the category $\mathrm{Lie}_{n} \mathrm{Alg}^{\mathrm{fin}}$ is taken to be a full subcategory of the category of conilpotent dg cocommutative coalgebras. For the purposes of the present paper, in order to make contact with Henriques' work [17], we now recall the dual picture. Let $(L, \ell) \in \mathrm{Lie}_{n} \mathrm{Alg}^{\text {fin }}$ be a finite type Lie $n$-algebra and $(\bar{S}(\mathbf{s} L), \delta)$ the associated $\mathrm{dg}$ coalgebra. We adjoin the ground field to obtain the corresponding coaugmented counital dg cocommutative coalgebra $(S(\mathbf{s} L), \delta)$, where $S(\mathbf{s} L)=\mathbb{R} \oplus \bar{S}(\mathbf{s} L)$ is the usual symmetric algebra and $\delta(1)=0$. (See [36, Sec. 2.4].)

We denote by

$$
\mathrm{CE}(L):=\left(S\left(\mathbf{s} L^{\vee}\right), \delta_{\mathrm{CE}}\right)
$$

the Chevalley-Eilenberg algebra of $(L, \ell)$ i.e. the $\mathbb{R}$-linear dual of $(S(\mathbf{s} L), \delta)$. It is naturally a unital commutative dg-algebra (cdga), whose differential $\delta_{\mathrm{CE}}:=\delta^{\vee}$ has degree 1. Since $(L, \ell)$ is finite type, $\operatorname{CE}(L)$ is semi-free. That is, its underlying commutative graded algebra is freely generated by the non-negatively graded vector space $\mathbf{s} L^{\vee}$, where $L_{i}^{\vee}:=\operatorname{hom}_{\mathbb{R}}\left(L_{i}, \mathbb{R}\right)$.

8.2.2. $\mathrm{Lie}_{n} \mathrm{Alg}^{\text {fin }}$ as a category of fibrant objects. As previously mentioned in Sec. 2 the only difference between our definition of an iCFO (2.1) and Brown's original definition of a category of fibrant objects is axiom 4. Brown requires the strong axiom that the pullback of a fibration always exists.

We summarize the main result of [36] in the following theorem:

Theorem 8.3 (Thm. 5.1 [36]). Let $n \in \mathbb{N} \cup\{\infty\}$. The category Lie $_{n}$ Alg $^{\text {fin }}$ of finite type Lie $n$-algebras and weak $L_{\infty}$-morphisms has the structure of a category of fibrant objects, in which a morphism $f:(L, \ell) \rightarrow\left(L^{\prime}, \ell\right)$ is:

- a weak equivalence iff it is a $L_{\infty}$-quasi-isomorphism (Def. 8.2),

- a fibration iff the chain map $f_{1}:\left(L, \ell_{1}\right) \rightarrow\left(L^{\prime}, \ell_{1}^{\prime}\right)$ is surjective in all positive degrees,

- an acyclic fibration iff $f$ is a $L_{\infty}$-quasi-isomorphism and the chain map $f_{1}:\left(L, \ell_{1}\right) \rightarrow\left(L^{\prime}, \ell_{1}^{\prime}\right)$ is surjective in all degrees.

We note that an explicit construction for path objects in $\mathrm{Lie}_{n} \mathrm{Alg}^{\text {fin }}$ is provided in [36, Sec 3.3].

Remark 8.4. Fibrations between Lie $n$-algebras, as defined in Thm. 8.3, coincide with fibrations in the projective model structure on non-negatively graded chain complexes. In contrast, the chain map $f_{1}$ associated to a fibration between unbounded $\mathbb{Z}$-graded $L_{\infty}$-algebras is required to be surjective in all degrees. (See for example [15], [35], and [39].)

In [37], Severa constructed a functor which provides a differentiation procedure Diff : $\mathrm{Lie}_{n} \mathrm{Grp}^{\mathrm{fin}} \rightarrow \mathrm{Lie}_{n} \mathrm{Alg}^{\text {fin }}$ from finite-dimensional Lie $n$-groups to Lie $n$-algebras. In certain cases, it is clear how this functor interacts with the iCFO structure (Thm. 7.1) on the category $\mathrm{Lie}_{n} \mathrm{Gpd}$, and this provides us with evidence that the notion of fibration given in Thm. 8.3 is the "correct" one for our applications. For example, suppose $\mathfrak{g}_{\bullet}$ is a simplicial Lie algebra, and let $G_{\bullet}$ denote the (level-wise) 
1-connected simplicial Lie group integrating $\mathfrak{g}_{\bullet}$. Then its classifying space $\bar{W} G_{\bullet}$ is a Lie $\infty$-group. Jurčo showed in $[23]$ that $\operatorname{Diff}\left(\bar{W} G_{\bullet}\right)$ is isomorphic to $N \mathfrak{g}_{\bullet}$, the dg Lie algebra obtained from $\mathfrak{g}_{\bullet}$ via Quillen's normalized chains functor [33]. Now suppose $f: \bar{W} G_{\bullet} \rightarrow \bar{W} G_{\bullet}^{\prime}$ is a Kan fibration between classifying spaces of 1-connected simplicial Lie groups in Lie ${ }_{\infty}$ Grp. Then, by using Jurčo's result, it is not difficult to see that $\operatorname{Diff}(f): N \mathfrak{g}_{\bullet} \rightarrow N \mathfrak{g}_{\bullet}^{\prime}$ is a dgla morphism that is surjective in all positive degrees, but not surjective, in general, in degree 0 . Therefore, if we hope to prove that Diff preserves fibrations, then the definition of fibration in the CFO structure on $\mathrm{Lie}_{n} \mathrm{Alg}^{\text {fin }}$ must be the one given in Thm. 8.3. A more detailed analysis of Ševera's functor is the subject of our future work.

8.3. Fibrations of Lie $n$-algebras. The following lemma from [36] is based on a result of Vallette [39] concerning the factorization of epimorphisms between $\mathbb{Z}$ graded homotopy algebras. The lemma allows us to dramatically simplify many constructions involving fibrations between Lie $n$-algebras. Roughly, it says that every fibration in $\mathrm{Lie}_{n} \mathrm{Alg}{ }^{\text {fin }}$ is a strict fibration up to isomorphism.

Lemma 8.5 (Lemma $3.11[36])$. Let $f:(L, \ell) \rightarrow\left(L^{\prime}, \ell^{\prime}\right)$ be a fibration between Lie $n$-algebras. Then there exists a Lie $n$-algebra $(L, \tilde{\ell})$ and an isomorphism $\phi:(L, \tilde{\ell}) \cong$ $(L, \ell)$ such that

$$
f \phi:(L, \tilde{\ell}) \rightarrow\left(L^{\prime}, \ell\right)
$$

is a strict fibration with $f \phi=(f \phi)_{1}=f_{1}$.

It turns out that not every fibration in $\mathrm{Lie}_{n} \mathrm{Alg}^{\mathrm{fin}}$ integrates to a fibration in $\mathrm{Lie}_{\infty}$ Grp (see Remark 9.11). However, we will show in Thm. 9.10 that there is a distinguished class of fibrations in $\mathrm{Lie}_{n} \mathrm{Alg}^{\text {fin }}$ that do. We call these "quasi-split fibrations".

Definition 8.6. A fibration of Lie $n$-algebras $f:(L, \ell) \rightarrow\left(L^{\prime}, \ell^{\prime}\right)$ is a quasi-split fibration iff

(1) the induced map in homology $H\left(f_{1}\right): H(L) \rightarrow H\left(L^{\prime}\right)$ is surjective in all degrees and,

(2) $H_{0}(L) \cong \operatorname{ker} H_{0}\left(f_{1}\right) \oplus H_{0}\left(L^{\prime}\right)$ in the category of Lie algebras.

Remark 8.7. Note that every acyclic fibration in $\mathrm{Lie}_{n} \mathrm{Alg}^{\text {fin }}$ is a quasi-split fibration. More generally, $f:(L, \ell) \rightarrow\left(L^{\prime}, \ell^{\prime}\right)$ is a quasi-split fibration if $\operatorname{ker} H\left(f_{1}\right)$ is central and $H\left(f_{1}\right): H(L) \rightarrow H\left(L^{\prime}\right)$ is a split epimorphism in the category of $H_{0}(L)$-modules.

Besides acyclic fibrations, there are other examples of quasi-split fibrations which naturally arise in interesting applications. In particular, the string Lie 2-algebra, whose integration was the original motivation for [17], is a special case of the following construction.

Example 8.8 (Central $n$-extensions). Let $(\mathfrak{g},[\cdot, \cdot])$ be a Lie algebra and $c: \Lambda^{n+1} \mathfrak{g} \rightarrow$ $\mathbb{R}$ a degree $n+1$ cocycle in the Chevalley-Eilenberg complex associated to $\mathfrak{g}$. From this data we obtain a Lie $n$-algebra $\widehat{\mathfrak{g}}_{c}$ whose underlying vector space is concentrated in degrees 0 and $n-1$ :

$$
\widehat{\mathfrak{g}}_{c}=\mathfrak{g} \oplus \mathbb{R}[1-n]
$$

and whose only non-trivial brackets are

$$
\begin{aligned}
\ell_{2}\left(x_{1}, x_{2}\right) & =\left[x_{1}, x_{2}\right], \quad \text { if } x_{1}, x_{2} \in \mathfrak{g} \\
\ell_{n+1}\left(x_{1}, \ldots, x_{n+1}\right) & =c\left(x_{1}, \ldots, x_{n+1}\right), \quad \text { if } x_{1}, \ldots, x_{n+1} \in \mathfrak{g}
\end{aligned}
$$


A straightforward verification shows that the linear projection $\pi: \widehat{\mathfrak{g}}_{c} \rightarrow \mathfrak{g}$ extends to a strict quasi-split fibration sequence $\mathbb{R}[1-n] \hookrightarrow \widehat{\mathfrak{g}}_{c} \stackrel{\pi}{\rightarrow} \mathfrak{g}$ in $\operatorname{Lie}_{n} \mathrm{Alg}^{\text {fin }}$.

We conclude this section with a corollary concerning the "strictification" of quasisplit fibrations. The proof follows directly from Lemma 8.5 and Def. 8.6.

Corollary 8.9. Let $f:(L, \ell) \rightarrow\left(L^{\prime}, \ell^{\prime}\right)$ be a quasi-split fibration between Lie $n$ algebras. Then there exists an isomorphism $\phi:(L, \tilde{\ell}) \cong(L, \ell)$ in $\operatorname{Lie}_{n} \mathrm{Alg}^{\mathrm{fin}}$ such that $f \phi:(L, \tilde{\ell}) \rightarrow\left(L^{\prime}, \ell\right)$ is a strict quasi-split fibration with $f \phi=(f \phi)_{1}=f_{1}$.

8.4. Postnikov tower for Lie $n$-algebras. Next, we recall the following technical results from [36, Sec. 7] concerning Postnikov towers. These will play a crucial role in our analysis of the integration functor in Sec. 9.

Let $(L, \ell)$ be a Lie $n$-algebra. Following [17, Def. 5.6], we consider two different truncations of the underlying chain complex $\left(L, d=\ell_{1}\right)$. For any $m \geq 0$, denote by $\tau_{\leq m} L$ and $\tau_{<m} L$ the following $(m+1)$-term complexes:

$$
\left(\tau_{\leq m} L\right)_{i}=\left\{\begin{array}{ll}
L_{i} & \text { if } i<m, \\
\operatorname{coker}\left(d_{m+1}\right) & \text { if } i=m, \\
0 & \text { if } i>m,
\end{array} \quad\left(\tau_{<m} L\right)_{i}= \begin{cases}L_{i} & \text { if } i<m, \\
\operatorname{im}\left(d_{m}\right) & \text { if } i=m, \\
0 & \text { if } i>m .\end{cases}\right.
$$

In degree $m$, the differentials for $\tau_{\leq m} L$ and $\tau_{<m} L$ are $d_{m}: L_{m} / \operatorname{im}\left(d_{m+1}\right) \rightarrow L_{m-1}$, and the inclusion $\operatorname{im}\left(d_{m}\right) \hookrightarrow L_{m-1}$, respectively. The homology complexes of $\tau_{\leq m} L$ and $\tau_{<m} L$ are

$$
H_{i}\left(\tau_{\leq m} L\right)=\left\{\begin{array}{ll}
H_{i}(L) & \text { if } i \leq m, \\
0 & \text { if } i>m,
\end{array} \quad H_{i}\left(\tau_{<m} L\right)= \begin{cases}H_{i}(L) & \text { if } i<m, \\
0 & \text { if } i \geq m .\end{cases}\right.
$$

We have the following obvious surjective chain maps

$$
p_{\leq m}: L \rightarrow \tau_{\leq m} L \quad p_{<m}: L \rightarrow \tau_{<m} L
$$

where in degree $m$, the map $p_{\leq m}$ is the surjection $L_{m} \rightarrow \operatorname{coker}\left(d_{m+1}\right)$, and $p_{<m}$ is the differential $d_{m}: L_{m} \rightarrow \operatorname{im}\left(d_{m}\right)$. There are also the similarly defined surjective chain maps

$$
q_{\leq m}: \tau_{\leq m} L \rightarrow \tau_{<m} L, \quad q_{<m+1}: \tau_{<m+1} L \stackrel{\sim}{\rightarrow} \tau_{\leq m} L .
$$

The map $q_{\leq m}$ in degree $m$ is the differential $d_{m}$ : coker $d_{m+1} \rightarrow \operatorname{im} d_{m}$, and the identity in all other degrees. The map $q_{<m+1}$ is the projection $L_{m} \rightarrow$ coker $d_{m+1}$ in degree $m$, the identity in all degrees $<m$, and the zero map in degree $m+1$. We note that $q_{<m+1}$ is a quasi-isomorphism of complexes.

Proposition 8.10 (Prop. $7.2[36]$ ). Let $(L, \ell)$ be a Lie n-algebra.

(1) The Lie $n$-algebra structure on $(L, \ell)$ induces Lie $(m+1)$-structures on the complexes $\tau_{\leq m} L$ and $\tau_{<m} L$ whose brackets are given by

$\tau_{\leq m} \ell_{k}\left(\bar{x}_{1}, \ldots, \bar{x}_{k}\right):=p_{\leq m} \ell_{k}\left(x_{1}, \ldots, x_{k}\right), \quad \tau_{<m} \ell_{k}\left(\bar{y}_{1}, \ldots, \bar{y}_{k}\right):=p_{<m} \ell_{k}\left(y_{1}, \ldots, y_{k}\right)$, where $\bar{x}_{i}=p_{\leq m}\left(x_{i}\right)$ and $\bar{y}_{i}=p_{<m}\left(y_{i}\right)$.

(2) The assignments $(L, \ell) \mapsto\left(\tau_{\leq m} L, \tau_{\leq m} \ell\right)$ and $(L, \ell) \mapsto\left(\tau_{<m} L, \tau_{<m} \ell\right)$ are functorial. 
(3) An $L_{\infty}$-morphism $f:(L, \ell) \rightarrow\left(L^{\prime}, \ell^{\prime}\right)$ induces a morphism of towers of Lie $n$-algebras

$$
\begin{aligned}
& \cdots \tau_{\leq m-1} L \stackrel{q_{\leq m-1}}{\longrightarrow} \tau_{<m-1} L \stackrel{q<m-1}{\longrightarrow} \tau_{\leq m-2} L \stackrel{q \leq m-2}{\longrightarrow} \cdots \longrightarrow \tau_{\leq 1} L \stackrel{q_{\leq 1}}{\longrightarrow} \tau_{<1} L \stackrel{q_{<1}}{\longrightarrow} \tau_{\leq 0} L \\
& \downarrow \tau_{<m-1} f\left|\quad \tau_{\leq m-2} f\right| \quad \tau_{\leq 1} f\left|\quad \tau_{<1} f\right| \quad \tau_{\leq 0} f \mid \\
& \cdots \tau_{\leq m-1} L_{q_{\leq m-1}^{\prime}}^{\longrightarrow \longrightarrow} \tau_{<m-1} L_{q_{<m-1}^{\prime}}^{\longrightarrow \longrightarrow} \tau_{\leq m-2} L_{q_{\leq m-2}^{\prime}}^{\longrightarrow} \cdots \longrightarrow \tau_{\leq 1} L^{\prime} \underset{q_{\leq 1}^{\prime}}{\longrightarrow} \tau_{<1} L^{\prime} \underset{q_{<1}^{\prime}}{\longrightarrow} \tau_{\leq 0} L^{\prime}
\end{aligned}
$$

in which the horizontal arrows are the strict $L_{\infty}$-morphisms induced by the surjective chain maps (8.3).

Remark 8.11. The vertical maps $\tau_{<m} f$ and $\tau_{\leq m} f$ in (8.4) induced by the morphism $f:(L, \ell) \rightarrow\left(L^{\prime}, \ell^{\prime}\right)$ are defined via the projections given in Eq. 8.2. For example,

$$
\tau_{\leq m} f_{k}\left(\bar{x}_{1}, \ldots, \bar{x}_{k}\right):=p_{\leq m}^{\prime} f_{k}\left(x_{1}, \ldots, x_{k}\right) .
$$

We also note that the Lie $n$-algebra $\tau_{\leq 0} L$ is just the Lie algebra $H_{0}(L)$ concentrated in degree zero. Given a morphism of Lie $n$-algebras $f:(L, \ell) \rightarrow\left(L^{\prime}, \ell^{\prime}\right)$, the induced morphism $\tau_{\leq 0} f: H_{0}(L) \rightarrow H_{0}\left(L^{\prime}\right)$ of Lie algebras is the morphism $H_{0}\left(f_{1}\right)$ from Remark 8.1.

8.5. Quasi-split fibrations and decomposition of towers. The commutative diagram (8.4) has a convenient decomposition in the case when $f:(L, \ell) \rightarrow\left(L^{\prime}, \ell^{\prime}\right)$ is a quasi-split fibration (8.6). In these next two results recalled from Sec. 7.2 of [36], we only consider strict quasi-split fibrations. The general case involving arbitrary quasi-split fibrations follows from applying Cor. 8.9.

Let ker $q_{<m+1}$ denote the kernel of the map $q_{<m+1}:\left(\tau_{<m+1} L, \tau_{<m+1} \ell\right) \rightarrow\left(\tau_{\leq m} L, \tau_{\leq m} \ell\right)$ defined in (8.3) Then the chain complex $\operatorname{ker} q_{<m+1}$ is an abelian Lie $n$-algebra concentrated in degrees $m$ and $m+1$ with

$$
\left(\operatorname{ker} q_{<m+1}\right)_{m}=\operatorname{im} d_{m+1}, \quad\left(\operatorname{ker} q_{<m+1}\right)_{m+1}=\operatorname{im} d_{m+1}[-1]
$$

The induced differential $\ell^{\text {ker }}=\ell_{1}$ on $\operatorname{ker} q_{<m+1}$ is simply the desuspension isomorphism.

Proposition 8.12 (Prop. $7.5[36])$. Let $f=f_{1}:(L, \ell) \rightarrow\left(L^{\prime}, \ell^{\prime}\right)$ be a strict quasisplit fibration. Then there exists morphisms in $\mathrm{Lie}_{n} \mathrm{Alg}^{\mathrm{fin}}$

$r:\left(\tau_{<m+1} L, \tau_{<m+1} \ell\right) \rightarrow\left(\operatorname{ker} q_{<m+1}, \ell^{\mathrm{ker}}\right), \quad r^{\prime}:\left(\tau_{<m+1} L^{\prime}, \tau_{<m+1} \ell^{\prime}\right) \rightarrow\left(\operatorname{ker} q_{<m+1}^{\prime}, \ell^{\prime \operatorname{ker}}\right)$

inducing $L_{\infty}$-isomorphisms

$$
\begin{aligned}
& \left(q_{<m+1}, r\right):\left(\tau_{<m+1} L, \tau_{<m+1} \ell\right) \stackrel{\cong}{\rightrightarrows}\left(\tau_{\leq m} L \oplus \operatorname{ker} q_{<m+1}, \tau_{\leq m} \ell \oplus \ell^{\mathrm{ker}}\right) \\
& \left(q_{<m+1}^{\prime}, r^{\prime}\right):\left(\tau_{<m+1} L^{\prime}, \tau_{<m+1} \ell^{\prime}\right) \cong\left(\tau_{\leq m} L^{\prime} \oplus \operatorname{ker} q_{<m+1}^{\prime}, \tau_{\leq m} \ell^{\prime} \oplus \ell^{\prime \text { ker }}\right)
\end{aligned}
$$

such that the following diagram commutes in $\mathrm{Lie}_{n} \mathrm{Alg}$ :

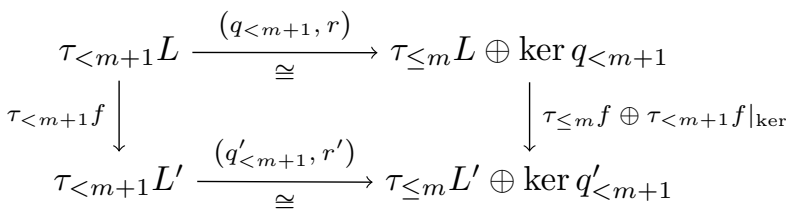


Now let $m \geq 1$. The chain map $q_{\leq m}$ defined in (8.3) induces a short exact sequence of chain complexes

$$
H_{m} \stackrel{i}{\rightarrow} \tau_{\leq m} L \stackrel{q_{\leq m}}{\rightarrow} \tau_{<m} L
$$

where $H_{m}$ is the homology group $H_{m}(L)$ concentrated in degree $m$ with trivial differential. The second decomposition result that we will need is:

Proposition 8.13 (Prop. $7.6[36])$. Let $m \geq 1$ and let $f=f_{1}:(L, \ell) \rightarrow\left(L^{\prime}, \ell^{\prime}\right)$ be a strict quasi-split fibration. Then there exists $L_{\infty}$-structures $\hat{\ell}$ and $\hat{\ell}^{\prime}$ on the graded vector spaces $\tau_{<m} L \oplus H_{m}$ and $\tau_{<m} L^{\prime} \oplus H_{m}^{\prime}$, respectively, and strict $L_{\infty}$ isomorphisms

$$
\begin{gathered}
\hat{q}:\left(\tau_{\leq m} L, \tau_{\leq m} \ell\right) \stackrel{\cong}{\longrightarrow}\left(\tau_{<m} L \oplus H_{m}, \hat{\ell}\right) \\
\hat{q}^{\prime}:\left(\tau_{\leq m} L^{\prime}, \tau_{\leq m} \ell^{\prime}\right) \stackrel{\cong}{\longrightarrow}\left(\tau_{<m} L^{\prime} \oplus H_{m}^{\prime}, \hat{\ell}^{\prime}\right)
\end{gathered}
$$

such that the following diagram of $L_{\infty}$-morphisms commutes

$$
\begin{array}{cll}
\left(\tau_{\leq m} L, \tau_{\leq m} \ell\right) \stackrel{\hat{q}}{\cong}\left(\tau_{<m} L \oplus H_{m}, \hat{\ell}\right) \\
\tau_{\leq m} f \mid & \downarrow \tau_{<m} f \oplus H(f) \\
\left(\tau_{\leq m} L^{\prime}, \tau_{\leq m} \ell^{\prime}\right) \stackrel{\hat{q}^{\prime}}{\cong}\left(\tau_{<m} L^{\prime} \oplus H_{m}^{\prime}, \hat{\ell}^{\prime}\right)
\end{array}
$$

Remark 8.14. In order to prove Thm. 9.10 in the next section, we'll need to recall from the proof of Prop. 7.6 in [36] some details concerning the isomorphisms $\hat{q}$ and $\hat{q}^{\prime}$. In degree $m$, the short exact sequence (8.7) and the analogous sequence for $q_{\leq m}^{\prime}$ gives the following commutative diagram of vector spaces.

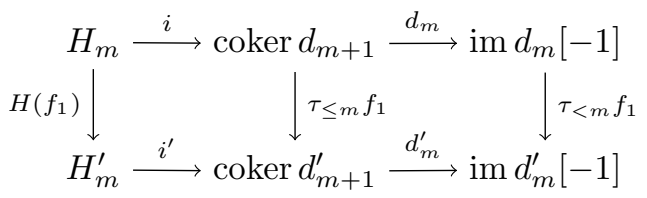

Since $f$ is a strict quasi-split fibration, there exists sections $s: \operatorname{im} d_{m}[-1] \rightarrow \operatorname{coker} d_{m+1}$, and $s^{\prime}: \operatorname{im} d_{m}^{\prime}[-1] \rightarrow$ coker $d_{m+1}^{\prime}$, of $d_{m}$ and $d_{m}^{\prime}$, respectively, such that $\tau_{\leq m} f_{1} \circ s=$ $s^{\prime} \circ \tau_{<m} f_{1}$. Let $t: \tau_{<m} L \rightarrow \tau_{\leq m} L$ and $r: \tau_{\leq m} L \rightarrow H_{m}$ be the linear maps

$$
t(x):=\left\{\begin{array}{ll}
s(x), & \text { if }|x|=m \\
x, & \text { if }|x|<m,
\end{array} \quad \hat{r}:=\mathrm{id}-t q_{\leq m}\right.
$$

respectively. Then the strict $L_{\infty}$-morphism $\hat{q}: \tau_{\leq m} L \rightarrow \tau_{<m} L \oplus H_{m}$ is defined to be

$$
\hat{q}(z):=\left(q_{\leq m}(z), \hat{r}(z)\right)
$$

The map $\hat{q}^{\prime}$ is defined in the analogous way, using the section $s^{\prime}$ instead of $s$.

Remark 8.15. As shown in Sec. 7.2.1 of [36], the structure maps $\hat{\ell}_{k}: \Lambda^{k}\left(\tau_{<m} L \oplus\right.$ $\left.H_{m}\right) \rightarrow \tau_{<m} L \oplus H_{m}$ for the $L_{\infty}$-structure on $\tau_{<m} L \oplus H_{m}$ are given by the formula:

$$
\begin{aligned}
& \hat{\ell}_{k}\left(\left(x_{1}, y_{1}\right),\left(x_{2}, y_{2}\right), \ldots,\left(x_{k}, y_{k}\right)\right)= \\
& \quad\left(\tau_{<m} \ell_{k}\left(x_{1}, x_{2}, \ldots, x_{k}\right), \hat{r} \circ \tau_{\leq m} \ell_{k}\left(t x_{1}+y_{1}, t x_{2}+y_{2}, \ldots, t x_{k}+y_{k}\right)\right),
\end{aligned}
$$


for all $x_{1}, \ldots, x_{k} \in \tau_{<m} L$ and $y_{1}, \ldots, y_{k} \in H_{m}$. The above formula implies that there is only one non-trivial structure map $\hat{\ell}_{k}$ that involves non-zero inputs from $H_{m}$, since $H_{m}$ is concentrated in top degree $m$. Namely:

$$
\hat{\ell}_{2}((x, 0),(0, y))=\left(0, \ell_{2}(x, y)\right)
$$

where $x \in L_{0}=\tau_{<m} L_{0}$ is an element of degree 0 and $y \in H_{m}$. Moreover, since $\ell_{2}$ satisfies the Leibniz rule with respect to the differential $\ell_{1}=d$, we obtain an action of the Lie algebra $H_{0}(L)=L_{0} / \operatorname{im} d_{1}$ on $H_{m}$ :

$$
\begin{array}{r}
H_{0}(L) \times H_{m} \rightarrow H_{m} \\
\left(x+\operatorname{im} d_{1}, y\right) \mapsto \ell_{2}(x, y)
\end{array}
$$

This observation will play a key role when we integrate quasi-split fibrations in Sec. 9 .

8.6. Maurer-Cartan elements. In Sec. 9, we express Henriques' integration functor in terms of Maurer-Cartan sets by applying some results from Sec. 6 of [36]. Throughout the present section, $S$ denotes a submanifold (possibly with corners) of $\mathbb{R}^{N}$, Fix an integer $r \geq 1$. Following [17, Sec. 5.1], we denote by

$$
\left(\Omega(S), d_{\mathrm{dR}}\right)
$$

the differential graded Banach algebra of " $r$-times continuously differentiable forms". By definition, a $k$-form $\alpha$ on $S$ is an element of $\Omega(S)$ if and only if both $\alpha$ and the $(k+1)$-form $d_{\mathrm{dR}} \alpha$ are $r$-times continuously differentiable.

Note that we treat $\left(\Omega(S), d_{\mathrm{dR}}\right)$ as a cochain complex with $d_{\mathrm{dR}}=\Omega(S)^{*} \rightarrow$ $\Omega(S)^{*+1}$ as usual. Let $\left(L, \ell_{k}\right) \in \operatorname{Lie}_{n} \mathrm{Alg}^{\text {fin }}$ be a finite type Lie $n$-algebra, and denote by

$$
\left(L \otimes \Omega(S), \ell^{\Omega}\right)
$$

the $\mathbb{Z}$-graded $L_{\infty}$-algebra whose underlying chain complex is $\left(L \otimes \Omega(S), \ell_{1}^{\Omega}\right)$ where

$$
\begin{aligned}
(L \otimes \Omega(S))_{m} & :=\bigoplus_{i-j=m} L_{i} \otimes \Omega(S)^{j} \\
\ell_{1}^{\Omega} & :=\ell_{1} \otimes \mathrm{id}_{\Omega(S)}+\mathrm{id}_{L} \otimes d_{\mathrm{dR}}
\end{aligned}
$$

and whose higher brackets are defined as:

$$
\ell_{k}^{\Omega}\left(x_{1} \otimes \omega_{1}, \ldots, x_{k} \otimes \omega_{k}\right):=(-1)^{\varepsilon} \ell_{k}\left(x_{1}, \ldots, x_{k}\right) \otimes \omega_{1} \omega_{2} \cdots \omega_{k},
$$

with

$$
\varepsilon:=\sum_{1 \leq i<j \leq k}\left|\omega_{i}\right|\left|x_{j}\right|
$$

It is easy to see that for every $(L, \ell) \in \operatorname{Lie}_{n} \mathrm{Alg}^{\text {fin }}$, the $L_{\infty}$-algebra $\left(L \otimes \Omega(S), \ell^{\Omega}\right)$ is "tame", in the sense of [36, Def. 6.1]. This means that the curvature of an element $a \in(L \otimes \Omega(S))_{-1}$ :

$$
\operatorname{curv}^{\Omega}(a)=\ell_{1}^{\Omega}(a)+\sum_{k \geq 2}(-1)^{\frac{k(k-1)}{2}} \frac{1}{k !} \ell_{k}^{\Omega}(a, a, \ldots, a) \in(L \otimes \Omega(S))_{-2}
$$

is well-defined, i.e., the above summation is finite. We recall that elements of the set

$$
\operatorname{MC}(L \otimes \Omega(S)):=\left\{a \in(L \otimes \Omega(S))_{-1} \mid \operatorname{curv}^{\Omega}(a)=0\right\} .
$$

are called the Maurer-Cartan elements of the $L_{\infty}$-algebra $\left(L \otimes \Omega(S), \ell^{\Omega}\right)$. 
Next, if $f:(L, \ell) \rightarrow\left(L^{\prime}, \ell^{\prime}\right)$ is a morphism of Lie $n$-algebras, then it is easy to verify that the maps $f_{k}^{\Omega}: \Lambda^{k}(L \otimes \Omega(S)) \rightarrow L^{\prime} \otimes \Omega(S)$ defined as

$$
f_{k}^{\Omega}\left(x_{1} \otimes \omega_{1}, \ldots, x_{k} \otimes \omega_{k}\right):=(-1)^{\varepsilon} f_{k}\left(x_{1}, \ldots, x_{k}\right) \otimes \omega_{1} \omega_{2} \cdots \omega_{k},
$$

assemble together to give a $L_{\infty}$-morphism $f^{\Omega}:\left(L \otimes \Omega(S), \ell^{\Omega}\right) \rightarrow\left(L^{\prime} \otimes \Omega(S), \ell^{\prime \Omega}\right)$. Furthermore, for any morphism $f:(L, \ell) \rightarrow\left(L^{\prime}, \ell^{\prime}\right)$ in $\mathrm{Lie}_{n} A \mathrm{Alg}^{\text {fin }}$, the $L_{\infty}$-morphism $f^{\Omega}:\left(L \otimes \Omega(S), \ell^{\Omega}\right) \rightarrow\left(L^{\prime} \otimes \Omega(S), \ell^{\prime \Omega}\right)$ is tame in the sense of [36, Def. 6.1]. This implies that the morphism $f^{\Omega}$ induces a well defined function

$$
f_{*}^{\Omega}:(L \otimes \Omega(S))_{-1} \rightarrow\left(L^{\prime} \otimes \Omega(S)\right)_{-1}
$$

where

$$
f_{*}^{\Omega}(a)=f_{1}^{\Omega}(a)+\sum_{k \geq 2}(-1)^{\frac{k(k-1)}{2}} \frac{1}{k !} f_{k}^{\Omega}(a, a, \ldots, a) .
$$

for all $a \in(L \otimes \Omega(S))_{-1}$. Note that if $f=f_{1}:(L, \ell) \rightarrow\left(L^{\prime}, \ell^{\prime}\right)$ is a strict morphism, then Eq. 8.12 implies that

$$
f_{*}^{\Omega}=f \otimes \operatorname{id}_{\Omega(S)}
$$

\section{Proposition 8.16.}

(1) If $f:(L, \ell) \rightarrow\left(L^{\prime}, \ell^{\prime}\right)$ is a morphism in $\mathrm{Lie}_{n} \mathrm{Alg}^{\text {fin }}$, then $f_{*}:(L \otimes \Omega(S))_{-1} \rightarrow$ $\left(L^{\prime} \otimes \Omega(S)\right)_{-1}$ restricts to a well-defined function

$$
f_{*}: \operatorname{MC}(L \otimes \Omega(S)) \rightarrow \operatorname{MC}\left(L^{\prime} \otimes \Omega(S)\right)
$$

between the corresponding Maurer-Cartan sets.

(2) The assignments $\left(L \otimes \Omega(S), \ell^{\Omega}\right) \mapsto \mathrm{MC}\left((L \otimes \Omega(S))\right.$, and $f^{\Omega} \mapsto f_{*}^{\Omega}$ define a functor

$$
\mathrm{MC}(-\otimes \Omega(S)): \operatorname{Lie}_{n} \mathrm{Alg}^{\mathrm{fin}} \rightarrow \text { Set },
$$

natural in $S \subseteq \mathbb{R}^{N}$.

(3) Let $(L, \ell) \in \mathrm{Lie}_{n} \mathrm{Alg}^{\text {fin }}$ and let $\mathrm{CE}(L)$ denote the Chevalley-Eilenberg algebra of $(L, \ell)$ defined in Sec. 8.2.1. Then the isomorphism of vector spaces

$$
\begin{array}{r}
\varphi: L \otimes \Omega(S) \stackrel{\cong}{\rightrightarrows} \operatorname{hom}_{\mathbb{R}}\left(\mathbf{s} L^{\vee}, \Omega(S)\right) \\
\varphi(x \otimes \omega)(f):=f(\mathbf{s} x) \omega
\end{array}
$$

extends to a bijection of sets

$$
\mathrm{MC}(L \otimes \Omega(S)) \stackrel{\cong}{\rightrightarrows} \operatorname{hom}_{\text {cdga }}(\mathrm{CE}(L), \Omega(S))
$$

natural in $(L, \ell) \in \operatorname{Lie}_{n} \mathrm{Alg}^{\text {fin }}$ and in $S \subseteq \mathbb{R}^{N}$.

Proof. Statements (1) and (2) follow from Prop. 6.3 and Lemma 6.4 in [36].

For (3), as mentioned in Sec. 8.2.1, $\mathrm{CE}(L)$ is freely generated as a graded commutative algebra by the vector space $\mathbf{s} L^{\vee}$. Hence, $\varphi$ induces an isomorphism $(L \otimes$ $\Omega(S))_{-1} \cong \operatorname{hom}_{\text {cga }}\left(\bar{S}\left(\mathbf{s} L^{\vee}\right), \Omega(S)\right)$. A direct calculation shows that $\operatorname{curv}^{\Omega}(x \otimes \omega)=0$ if and only if $\varphi(x \otimes \omega) \circ \delta_{\mathrm{CE}}=d_{\mathrm{dR}} \circ \varphi(x \otimes \omega)$. 


\section{INTEGRATION OF LIE $n$-ALGEBRAS}

9.1. From Lie $n$-algebras to Lie $\infty$-groups. A Lie $\infty$-group is an $\infty$-group object (Def. 3.4) in (Mfd, $\mathcal{T}_{\text {subm }}$ ). We denote by $\mathrm{Lie}_{\infty} \mathrm{Grp} \subseteq \mathrm{Lie}_{\infty} \mathrm{Gpd}$ the full subcategory of Lie $\infty$-groups. Let us recall Henriques' construction of Lie $\infty$-groups from Lie $n$-algebras of finite type. Recall that we denote by $\Omega\left(\Delta^{n}\right)$ the differential graded Banach algebra of " $r$-times continuously differentiable forms" (as defined in Sec. 8.6) on the geometric $n$-simplex.

Proposition-Definition 9.1 (Def. 5.2, Thm. 5.10 [17]). Let $L \in \operatorname{Lie}_{n} \mathrm{Alg}^{\text {fin }}$ be a finite type Lie n-algebra. The assignment

$$
L \mapsto\left(\int L\right)_{m}:=\operatorname{MC}\left(L \otimes \Omega\left(\Delta^{n}\right)\right) \cong \operatorname{hom}_{\text {cdga }}\left(\mathrm{CE}(L), \Omega\left(\Delta^{m}\right)\right)
$$

induces a functor

$$
\int: \mathrm{Lie}_{n} \mathrm{Alg}^{\mathrm{fin}} \rightarrow \mathrm{Lie}_{\infty} \mathrm{Grp}
$$

from the category of finite type Lie $n$-algebras to the category of Lie $\infty$-groups.

Remark 9.2. In [38], Ševera and Širaň prove that $\int L$ is a simplicial Banach manifold using an approach that differs from the one taken by Henriques' in his proof of [17, Thm. 5.10]. If $(L, \ell) \in \operatorname{Lie}_{n} \mathrm{Alg}^{\mathrm{fin}}$, then since $L$ is finite type, the Banach algebra structure on $\Omega\left(\Delta^{m}\right)$ naturally makes $L \otimes \Omega\left(\Delta^{m}\right)$ into a graded Banach space. Then Prop. 4.3 of [38] implies that $\operatorname{MC}\left(L \otimes \Omega\left(\Delta^{m}\right)\right) \subseteq\left(L \otimes \Omega\left(\Delta^{m}\right)_{-1}\right.$ is a Banach submanifold in the sense of $[25$, Ch. I,3]. From Eq. 8.11, we see that the curvature $\operatorname{curv}^{\Omega}:\left(L \otimes \Omega\left(\Delta^{m}\right)\right)_{-1} \rightarrow\left(L \otimes \Omega\left(\Delta^{m}\right)\right)_{-2}$ is a polynomial function and hence smooth. In other words,

$$
\operatorname{MC}\left(L \otimes \Omega\left(\Delta^{m}\right)\right) \longleftrightarrow\left(L \otimes \Omega\left(\Delta^{m}\right)\right)_{-1} \stackrel{\text { curv }^{\Omega}}{\longrightarrow}\left(L \otimes \Omega\left(\Delta^{m}\right)\right)_{-2}
$$

is an equalizer diagram in the category Mfd of Banach manifolds.

Before we proceed further, let us recall a very useful example of a Lie $\infty$-group stemming from the integration of a Lie $n$-algebra.

Example 9.3. Following [17, Example 5.5], let $(L, \ell) \in \operatorname{Lie}_{n} \operatorname{Alg}^{\text {fin }}$ and recall from Remark 8.11 that $\tau_{\leq 0} L=H_{0}(L)$ is a Lie algebra. Consider the Lie $\infty$-group $\int \tau_{\leq 0} L$. For each $m \geq 0$, we have a natural identification

$$
\left(\int \tau_{\leq 0} L\right)_{m}=\operatorname{MC}\left(\tau_{\leq 0} L \otimes \Omega^{*}\left(\Delta^{m}\right)\right) \cong\left(\tau_{\leq 0} L \otimes \Omega^{1}\left(\Delta^{m}\right)\right)^{b}
$$

between Maurer-Cartan elements and flat connections on the trivial bundle $G \times$ $\Delta^{m} \rightarrow \Delta^{m}$, where $G$ is the 1 -connected Lie group integrating $\tau_{\leq 0} L$. There is also an identification

$$
\begin{aligned}
T: \operatorname{Map}\left(\Delta^{m}, G\right) / G & \cong(\tau \leq 0 \\
\Delta^{m} & \left.\stackrel{\Gamma}{\rightarrow} G \quad \mapsto \quad T(\Gamma):=\Omega^{1}\left(\Delta^{m}\right)\right)^{b}
\end{aligned}
$$

between the set $\operatorname{Map}\left(\Delta^{m}, G\right) / G$ of $G$-valued $C^{r+1}$ maps, modulo constants, and the set of flat connections. This identification is natural in $(L, \ell)$. See Lemma 9.13. 
Remark 9.4. Note that the integration functor (9.1) a priori assigns to a Lie $n$ algebra not a Lie $n$-group but a Lie $\infty$-group. To resolve this, Henriques introduced a truncation functor [17, Def. 3.5] from Lie $\infty$-groups to simplical sheaves over Mfd. Furthermore, Henriques observed that, in general, there are obstructions to representing the output of the truncation functor as an actual Lie $n$-group. Further discussion of the truncation functor within the context of the homotopy theory of Lie $n$-groups will appear in future work.

We conclude this section with a warm-up result concerning the preservation of pullback squares by the integration functor (9.1). We address this in further detail in Thm. 9.16.

Proposition 9.5. Let $f:(L, \ell) \rightarrow\left(L^{\prime \prime}, \ell^{\prime \prime}\right)$ be a fibration and $g:\left(L^{\prime}, \ell^{\prime}\right) \rightarrow\left(L^{\prime \prime}, \ell^{\prime \prime}\right)$ be a morphism in $\mathrm{Lie}_{n} \mathrm{Alg}^{\text {fin }}$, and let $(\tilde{L}, \tilde{\ell})$ be the pullback of the diagram $\left(L^{\prime}, \ell^{\prime}\right) \stackrel{g}{\rightarrow}$ $\left(L^{\prime \prime}, \ell^{\prime \prime}\right) \stackrel{f}{\leftarrow}\left(L^{\prime \prime}, \ell^{\prime \prime}\right)$. If the pullback of

$$
\mathrm{MC}\left(L^{\prime} \otimes \Omega^{*}\left(\Delta^{m}\right)\right) \stackrel{g_{*}^{\Omega}}{\longrightarrow} \mathrm{MC}\left(L^{\prime \prime} \otimes \Omega^{*}\left(\Delta^{m}\right)\right) \stackrel{f_{*}^{\Omega}}{\longleftarrow} \mathrm{MC}\left(L \otimes \Omega^{*}\left(\Delta^{m}\right)\right)
$$

exists in the category $\mathrm{Mfd}$, then the induced commutative diagram

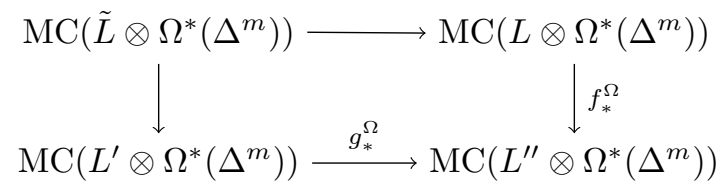

is a pullback diagram in Mfd.

Proof. Remark 9.2 implies that the Maurer-Cartan set $\operatorname{MC}\left(L \otimes \Omega^{*}\left(\Delta^{m}\right)\right)$ is an equalizer in Mfd for any $(L, \ell) \in \mathrm{Lie}_{n} \mathrm{Alg}^{\text {fin }}$. Therefore Assumption 6.6 in Sec. 6.2 of [36] is satisfied, and the proposition then follows from [36, Cor. 6.7].

We will use the following corollary in our proof of Thm. 9.10 in the next section.

Corollary 9.6. The integration functor $\int: \mathrm{Lie}_{n} \mathrm{Alg}^{\mathrm{fin}} \rightarrow \mathrm{Lie}_{\infty} \mathrm{Grp}$ preserves products.

Proof. The category Lie $\operatorname{Lrp}_{\infty}$ has finite products and every trivial morphism $(L, \ell) \rightarrow$ 0 in $\mathrm{Lie}_{n} \mathrm{Alg}^{\text {fin }}$ is a fibration.

9.2. Integrating fibrations. We begin by analyzing the naturality of a construction used by Henriques in his proof of the following result:

Proposition 9.7 (Thm. $5.10[17])$. Let $(L, \ell) \in \mathrm{Lie}_{n} \mathrm{Alg}^{\text {fin }}$. The integrations of the $L_{\infty}$-morphisms $q_{\leq m}$ and $q_{<m+1}$ defined in (8.3):

$$
\int q_{\leq m}: \int \tau_{\leq m} L \rightarrow \int \tau_{<m} L, \quad \int q_{<m+1}: \int \tau_{<m+1} L \rightarrow \int \tau_{\leq m} L
$$

are fibrations between Lie $\infty$-groups.

In what follows, the submanifold

$$
S \subseteq \mathbb{R}^{k+1}
$$

denotes either the geometric simplex $\Delta^{k}$ or a geometric horn $\Lambda_{j}^{k} \subseteq \Delta^{k}$. 
Let us fix a Lie $n$-algebra $(L, \ell) \in \operatorname{Lie}_{n} \mathrm{Alg}^{\text {fin }}$ and an integer $m \geq 1$. Our goal is to express elements of $\mathrm{MC}\left(\tau_{\leq m} L \otimes \Omega(S)\right)$ as pairs consisting of an element of $\mathrm{MC}\left(\tau_{<m} L \otimes \Omega(S)\right)$ and a $H_{m}(L)$-valued differential form. Via the strict $L_{\infty}$-isomorphism

$$
\hat{q}:\left(\tau_{\leq m} L, \tau_{\leq m} \ell\right) \stackrel{\cong}{\rightrightarrows}\left(\tau_{<m} L \oplus H_{m}, \hat{\ell}\right)
$$

given in Prop. 8.13, we have the identification

$$
\begin{aligned}
\operatorname{MC}\left(\tau_{\leq m} L \otimes \Omega(S)\right) \cong & \operatorname{MC}\left(\left(\tau_{<m} L \oplus H_{m}\right) \otimes \Omega(S)\right) \\
& \subseteq\left(\tau_{<m} L \otimes \Omega(S)\right)_{-1} \oplus H_{m} \otimes \Omega^{m+1}(S),
\end{aligned}
$$

where $H_{m}=H_{m}(L)$. Let $\widehat{\operatorname{curv}}^{\Omega}, \operatorname{curv}_{\leq m}^{\Omega}$ and $\operatorname{curv}_{<m}^{\Omega}$ denote the curvature functions (8.11) for the $L_{\infty}$-algebras

$$
\left(\left(\tau_{<m} L \oplus H_{m}\right) \otimes \Omega(S), \hat{\ell}^{\Omega}\right), \quad\left(\tau_{\leq m} L \otimes \Omega(S), \tau_{\leq m} \ell^{\Omega}\right), \quad\left(\tau_{<m} L \otimes \Omega(S), \tau_{<m} \ell^{\Omega}\right),
$$

respectively.

Since the $L_{\infty}$-isomorphism $\hat{q}^{\Omega}=\hat{q} \otimes \operatorname{id}_{\Omega(S)}$ is strict, we can easily write $\widehat{\mathrm{curv}} \Omega$ in terms of the other two curvature functions above. Indeed, consider a degree -1 element $(\sigma, \nu) \in\left(\tau_{<m} L \otimes \Omega(S)\right)_{-1} \oplus H_{m} \otimes \Omega^{m+1}(S)$. We first express its curvature as the sum of degree -2 elements: $\widehat{\operatorname{curv}}^{\Omega}(\sigma, \nu)=\sum_{i=1}^{m} \widehat{\operatorname{curv}}^{\Omega}(\sigma, \nu)_{i}$ where

$$
\widehat{\operatorname{curv}}^{\Omega}(\sigma, \nu)_{i} \in \tau_{<m} L_{i} \otimes \Omega^{i+2}(S) \text { for } i<m
$$

and

$$
\widehat{\operatorname{curv}}^{\Omega}(\sigma, \nu)_{m} \in \tau_{<m} L_{m} \otimes \Omega^{m+2}(S) \oplus H_{m} \otimes \Omega^{m+2}(S) .
$$

We then use formula (8.9) for the $L_{\infty}$-structure on $\left(\tau_{<m} L \oplus H_{m}\right)$ to obtain the equality

$$
\widehat{\operatorname{curv}} \Omega^{\Omega}(\sigma, \nu)_{i}= \begin{cases}\left(\operatorname{curv}_{<m}^{\Omega}(\sigma)_{i}, 0\right) & \text { if } i<m \\ \left(\operatorname{curv}_{<m}^{\Omega}(\sigma)_{m}, d_{\mathrm{dR}} \nu-\ell_{2}^{\Omega}\left(\sigma_{0}, \nu\right)-\kappa(\sigma)\right), & \text { if } i=m .\end{cases}
$$

Above $\sigma_{0} \in L_{0} \otimes \Omega^{1}(S)$ is the component of $\sigma$ in bidegree $(0,-1)$ and $\kappa(\sigma)$ denotes the $H_{m}$-valued $(m+2)$-form

$$
\kappa(\sigma):=-\left(\operatorname{pr}_{H_{m}} \otimes \operatorname{id}_{\Omega(S)}\right) \circ \hat{q}^{\Omega} \circ \operatorname{curv}_{\leq m}^{\Omega} \circ\left(\hat{q}^{\Omega}\right)^{-1}(\sigma, 0) \in H_{m} \otimes \Omega^{m+2}(S),
$$

where $\operatorname{pr}_{H_{m}}: \tau_{<m} L \oplus H_{m} \rightarrow H_{m}$ is the linear projection.

Hence, we have the following characterization of the Maurer-Cartan elements:

$$
\begin{aligned}
& \mathrm{MC}\left(\tau_{\leq m} L \otimes \Omega(S)\right)= \\
& \left\{(\sigma, \nu) \in \mathrm{MC}\left(\tau_{<m} L \otimes \Omega(S)\right) \oplus H_{m} \otimes \Omega^{m+1}(S) \mid d_{\mathrm{dR}} \nu-\ell_{2}^{\Omega}\left(\sigma_{0}, \nu\right)=\kappa(\sigma)\right\} .
\end{aligned}
$$

Now suppose $\sigma$ is a Maurer-Cartan element of $\tau_{<m} L \otimes \Omega(S)$ and $\nu \in H_{m} \otimes$ $\Omega^{m+1}(S)$ is a $H_{m}$-valued $(m+1)$-form. As observed by Henriques in his proof of [17, Thm. 5.10$]$, the condition

$$
d_{\mathrm{dR}} \nu-\ell_{2}^{\Omega}\left(\sigma_{0}, \nu\right)=\kappa(\sigma),
$$

can be rewritten by exploiting the action (8.10) of the Lie algebra $H_{0}(L)$ on $H_{m} \otimes$ $\Omega^{*}(S)$. We do this in the following way: Let $\alpha \in H_{0}(L) \otimes \Omega^{1}(S)$ denote the class represented by $\sigma_{0}$. Since $\operatorname{curv}_{<m}^{\Omega}(\sigma)=0$, we have $d_{\mathrm{dR}} \alpha-\frac{1}{2}[\alpha, \alpha]=0$, where $[\cdot, \cdot]$ denotes the Lie bracket on $H_{0}(L) \otimes \Omega(S)$ induced by $\ell_{2}^{\Omega}$. Hence, $\alpha$ is a flat 
$H_{0}(L)$-valued connection on $S$. Furthermore, since $\ell_{2}$ satisfies the Leibniz rule, one observes that $(\sigma, \nu)$ satisfies Eq. 9.4 if and only if

$$
d_{\mathrm{dR}} \nu-[\alpha, \nu]=\kappa(\sigma) .
$$

Let $G$ be the 1-connected Lie group integrating $H_{0}(L)$. Then $H_{m} \otimes \Omega^{*}(S)$ integrates to a $G$-module in the standard way. Via the identification (9.2) introduced in Example 9.3, there exists a unique function $\Gamma: S \rightarrow G$ such that

$$
-\alpha=-\Gamma^{-1} d \Gamma, \quad \Gamma\left(v_{0}\right)=e,
$$

where $v_{0}$ is an arbitrary fixed vertex of $S$. Then, as shown in [17, Eqs. 30-32], $(\sigma, \nu)$ satisfy Eq. 9.5 if and only if

$$
d_{\mathrm{dR}}(\Gamma \cdot \nu)=\Gamma \cdot \kappa(\sigma) .
$$

This characterization of Maurer-Cartan elements using the $H_{0}(L)$ action is natural in the following sense:

Proposition 9.8. Let $S=\Lambda_{j}^{k}$ or $\Delta^{k}$ and $m \geq 1$. Suppose $f=f_{1}:(L, \ell) \rightarrow\left(L^{\prime}, \ell^{\prime}\right)$ is a strict quasi-split fibration in $\mathrm{Lie}_{n} \mathrm{Alg}^{\text {fin }}$. Let

$$
\begin{aligned}
& \operatorname{MC}\left(\tau_{\leq m} L \otimes \Omega(S)\right) \underset{\cong}{\stackrel{\hat{q}_{*}^{\Omega}}{\cong}} \operatorname{MC}\left(\left(\tau_{<m} L \oplus H_{m}\right) \otimes \Omega(S)\right) \\
& \tau_{\leq m} f_{*}^{\Omega} \downarrow \quad \downarrow \tau_{<m} f_{*}^{\Omega} \oplus H(f) \otimes \mathrm{id}_{\Omega} \\
& \operatorname{MC}\left(\tau_{\leq m} L^{\prime} \otimes \Omega(S)\right) \underset{\hat{q}_{*}^{\prime \Omega}}{\cong} \operatorname{MC}\left(\left(\tau_{<m} L^{\prime} \oplus H_{m}^{\prime}\right) \otimes \Omega(S)\right)
\end{aligned}
$$

be the commutative diagram of smooth manifolds induced by diagram (8.8) in Prop. 8.13. If $a \in \mathrm{MC}\left(\tau_{\leq m} L \otimes \Omega(S)\right)$ is a Maurer-Cartan element and we define

$$
(\sigma, \nu):=\hat{q}_{*}^{\Omega}(a), \quad\left(\sigma^{\prime}, \nu^{\prime}\right):=\hat{q}_{*}^{\prime \Omega} \circ \tau_{\leq m} f_{*}^{\Omega}(a),
$$

then we have the following equalities of $H_{m}^{\prime}$-valued differential forms:

$$
\begin{aligned}
\left(H(f) \otimes \operatorname{id}_{\Omega(S)}\right)(\Gamma \cdot \nu) & =\Gamma^{\prime} \cdot \nu^{\prime} \\
\left(H(f) \otimes \operatorname{id}_{\Omega(S)}\right)(\Gamma \cdot \kappa(\sigma)) & =\Gamma^{\prime} \cdot \kappa^{\prime}\left(\sigma^{\prime}\right),
\end{aligned}
$$

where $\Gamma^{\prime}: S \rightarrow G^{\prime}$ is the unique integration defined in Eq. 9.6 of the flat $H_{0}\left(L^{\prime}\right)$ valued connection induced by $\sigma_{0}^{\prime} \in L_{0}^{\prime} \otimes \Omega^{1}(S)$, and $\kappa^{\prime}\left(\sigma^{\prime}\right)$ is the differential form defined via Eq. 9.3.

Proof. Since $f=f_{1}$ is a strict $L_{\infty}$-morphism, it follows from the commutativity of diagram (9.8) and the formula for $\tau_{<m} f_{*}^{\Omega}$ (Eq. 8.13) that:

$$
\left(\sigma^{\prime}, \nu^{\prime}\right)=\left(\left(\tau_{<m} f \otimes \operatorname{id}_{\Omega(S)}\right)(\sigma),\left(H(f) \otimes \operatorname{id}_{\Omega(S)}\right)(\nu)\right) .
$$

In particular, $\sigma_{0}^{\prime}=\left(f \otimes \operatorname{id}_{\Omega(S)}\right)\left(\sigma_{0}\right)$. If $\Gamma: S \rightarrow G$ is the unique integration (9.6) of the flat $H_{0}(L)$-valued connection induced by $\sigma_{0}$, then, by uniqueness, $\Gamma^{\prime}=\Phi \circ \Gamma$, where $\Phi: G \rightarrow G^{\prime}$ is the Lie group homomorphism integrating $H(f): H_{0}(L) \rightarrow$ $H_{0}\left(L^{\prime}\right)$. The linear map $H(f) \otimes \operatorname{id}_{\Omega(S)}: H_{m} \otimes \Omega(S) \rightarrow H_{m}^{\prime} \otimes \Omega(S)$ intertwines the Lie algebra actions, since $f$ is a strict $L_{\infty}$-morphism. Hence, it also intertwines the Lie group actions:

$$
\left(H(f) \otimes \operatorname{id}_{\Omega(S)}\right)(\Gamma \cdot \nu)=(\Phi \circ \Gamma) \cdot \nu^{\prime}=\Gamma^{\prime} \cdot \nu^{\prime} .
$$


HOMOTOPY THEORY FOR LIE $\infty$-GROUPOIDS \& APPLICATION TO INTEGRATION 55

Similarly, we have

$$
\left(H(f) \otimes \operatorname{id}_{\Omega(S)}\right)(\Gamma \cdot \kappa(\sigma))=\Gamma^{\prime} \cdot\left(H(f) \otimes \operatorname{id}_{\Omega(S)}\right)(\kappa(\sigma)) .
$$

It follows from the definition of $\kappa(\sigma)$ in Eq. 9.3 that:

$$
\left(H(f) \otimes \operatorname{id}_{\Omega(S)}\right)(\kappa(\sigma))=\left(\left(H(f) \operatorname{pr}_{H_{m}} \hat{q}\right) \otimes \operatorname{id}_{\Omega(S)} \circ \operatorname{curv}_{\leq m}^{\Omega} \circ\left(\hat{q}^{-1} \otimes \operatorname{id}_{\Omega(S)}\right)(\sigma, 0) .\right.
$$

The commutative diagram (8.8) implies that $H(f) \circ \operatorname{pr}_{H_{m}} \hat{q}=\operatorname{pr}_{H_{m}^{\prime}} \hat{q}^{\prime} \circ \tau_{\leq m} f$. Since $\tau_{\leq m} f$ is a strict $L_{\infty}$-morphism, we have

$$
\left(\tau_{\leq m} f \otimes \operatorname{id}_{\Omega(S)}\right) \circ \operatorname{curv}_{\leq m}^{\Omega}=\operatorname{curv}_{\leq m}^{\prime \Omega} \circ\left(\tau_{\leq m} f \otimes \operatorname{id}_{\Omega(S)}\right)
$$

where $\operatorname{curv}_{\leq m}^{\prime \Omega}$ is the curvature function for the $L_{\infty}$-algebra $\tau_{\leq m} L^{\prime} \otimes \Omega(S)$. Hence, we obtain the following equalities:

$$
\begin{aligned}
\left(H(f) \otimes \operatorname{id}_{\Omega(S)}\right)(\kappa(\sigma))= & \left(\operatorname{pr}_{H_{m}^{\prime}} \hat{q}^{\prime} \otimes \operatorname{id}_{\Omega(S)}\right) \operatorname{curv}_{\leq m}^{\prime \Omega} \circ\left(\tau_{\leq m} f \otimes \operatorname{id}_{\Omega(S)}\right)\left(\hat{q}^{-1} \otimes \operatorname{id}_{\Omega(S)}\right)(\sigma, 0) \\
= & \left(\operatorname{pr}_{H_{m}^{\prime}} \hat{q}^{\prime} \otimes \operatorname{id}_{\Omega(S)}\right) \operatorname{curv}_{\leq m}^{\prime \Omega} \circ\left(\hat{q}^{\prime-1} \otimes \operatorname{id}_{\Omega(S)}\right) \circ \\
& \quad\left(\left(\tau_{<m} f \oplus H(f)\right) \otimes \operatorname{id}_{\Omega(S)}\right)(\sigma, 0) \\
= & \left(\operatorname{pr}_{H_{m}^{\prime}} \hat{q}^{\prime} \otimes \operatorname{id}_{\Omega(S)}\right) \operatorname{curv}_{\leq m}^{\prime \Omega} \circ\left(\hat{q}^{\prime-1} \otimes \operatorname{id}_{\Omega(S)}\right)\left(\sigma^{\prime}, 0\right) \\
= & \kappa^{\prime}\left(\sigma^{\prime}\right) .
\end{aligned}
$$

By combining this last equality with Eq. 9.9, we conclude that

$$
\left(H(f) \otimes \operatorname{id}_{\Omega(S)}\right)(\Gamma \cdot \kappa(\sigma))=\Gamma^{\prime} \cdot \kappa^{\prime}\left(\sigma^{\prime}\right) .
$$

Corollary 9.9. Let $m \geq 1$ and suppose $f=f_{1}:(L, \ell) \rightarrow\left(L^{\prime}, \ell^{\prime}\right)$ is a strict quasisplit fibration as in Prop. 9.8. Let

$$
\begin{aligned}
X(S) & :=\left\{(\sigma, \rho) \in \mathrm{MC}\left(\tau_{<m} L \otimes \Omega(S)\right) \times\left(H_{m} \otimes \Omega^{m+1}(S)\right) \mid d_{\mathrm{dR}} \rho=\gamma(\sigma)\right\} \\
X^{\prime}(S) & :=\left\{\left(\sigma^{\prime}, \rho^{\prime}\right) \in \operatorname{MC}\left(\tau_{<m} L^{\prime} \otimes \Omega(S)\right) \times\left(H_{m}^{\prime} \otimes \Omega^{m+1}(S)\right) \mid d_{\mathrm{dR}} \rho^{\prime}=\gamma^{\prime}\left(\sigma^{\prime}\right)\right\},
\end{aligned}
$$

where $\gamma(\sigma)$ and $\gamma^{\prime}\left(\sigma^{\prime}\right)$ denote the $(m+2)$-forms $\Gamma \cdot \kappa(\sigma)$ and $\Gamma^{\prime} \cdot \kappa^{\prime}\left(\sigma^{\prime}\right)$, respectively, as in Prop. 9.8. Then the following diagram of smooth manifolds commutes:

$$
\begin{gathered}
\operatorname{MC}\left(\left(\tau_{<m} L \oplus H_{m}\right) \otimes \Omega(S)\right) \stackrel{\left(\mathrm{id}_{\tau_{<m} L \otimes \Omega(S)}, \Gamma \cdot-\right)}{\cong} X(S) \\
\tau_{<m} f_{*}^{\Omega} \oplus\left(H(f) \otimes \mathrm{id}_{\Omega}\right) \downarrow \\
\operatorname{MC}\left(\left(\tau_{<m} L^{\prime} \oplus H_{m}^{\prime}\right) \otimes \Omega(S)\right) \stackrel{\left(\mathrm{id}_{\tau_{<m} L^{\prime} \otimes \Omega(S)}, \Gamma^{\prime} \cdot-\right)}{\cong} X^{\prime}(S)
\end{gathered}
$$

Proof. Given a finite-dimensional $G$-module $H$ and a smooth map $g: S \rightarrow G$, the linear map

$$
H \otimes \Omega(S) \stackrel{g \cdot-}{\longrightarrow} H \otimes \Omega(S), \quad \nu \mapsto g \cdot \nu
$$

is a smooth isomorphism between Banach spaces. The rest of the corollary then follows from Prop. 9.8 and Eq. 9.7.

We now prove the main result of this section. 
Theorem 9.10. Let $f:(L, \ell) \rightarrow\left(L^{\prime}, \ell^{\prime}\right)$ be a quasi-split fibration in $\mathrm{Lie}_{n} \mathrm{Alg}^{\mathrm{fin}}$. Then the simplicial map

$$
\int f: \int L \rightarrow \int L^{\prime}
$$

is a fibration between Lie $\infty$-groups.

Proof. Corollary 8.9 implies that we can factor any quasi-split fibration into an $L_{\infty}$-isomorphism, followed by a strict quasi-split fibration. Therefore, in order to prove the theorem, we only need to consider the case when $f$ is strict.

Let $f=f_{1}:(L, \ell) \rightarrow\left(L^{\prime}, \ell^{\prime}\right)$ be a strict quasi-split fibration. Let $k>0$ and $0 \leq j \leq k$. It is straightforward to show that $\int f$ satisfies the Kan condition $\operatorname{Kan}(k, j)$ if and only if, for $m \geq k+1$, the integration of the truncated $L_{\infty^{-}}$ morphism

$$
\int \tau_{\leq m} f: \int\left(\tau_{\leq m} L, \tau_{\leq m} \ell\right) \rightarrow \int\left(\tau_{\leq m} L^{\prime}, \tau_{\leq m} \ell^{\prime}\right)
$$

satisfies $\operatorname{Kan}(k, j)$. Hence, it suffices to show that every vertical map appearing in the morphism of Postnikov towers (8.4) integrates to a fibration between Lie $\infty$-groups.

We proceed by induction on the truncation level $m$. For the base case, we need to verify that $\int \tau_{\leq 0} L \rightarrow \int \tau_{\leq 0} L^{\prime}$ is a fibration. By definition of the truncation functor $\tau_{\leq 0}$, this is equivalent to verifying that Lie algebra morphism $H(f): H_{0}(L) \rightarrow$ $H_{0}\left(L^{\prime}\right)$ integrates to a fibration between Lie $\infty$-groups of the type discussed in Example 9.2. The hypothesis on $H_{0}(f)$ implies that $H_{0}(L)$ is the trivial extension of $H_{0}\left(L^{\prime}\right)$ by ker $H_{0}(f)$. Therefore, we have the following commutative diagram of Lie algebras

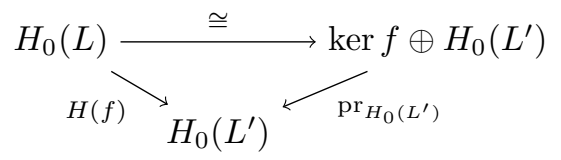

Corollary 9.6 implies that the integration functor preserves products. Since ker $f \oplus$

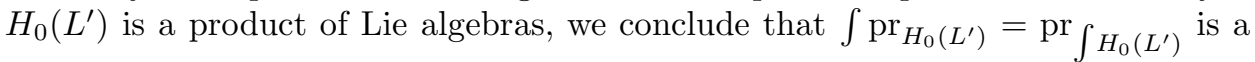
fibration, and hence, $\int H(f)$ is a fibration as well. This completes the base case.

The induction step involves two cases.

Case 1: First, let $m \geq 0$ and consider the following commutative diagram in $\operatorname{Lie}_{n}$ Alg $^{\text {fin }}$ :

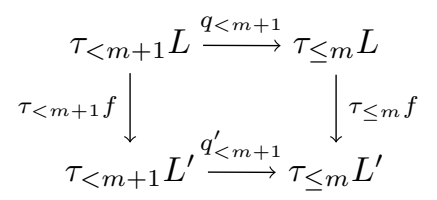

Suppose that the morphism $\int \tau_{\leq m} f$ is a fibration in Lie ${ }_{\infty}$ Grp. We will show that $\int \tau_{<m+1} f$ is also a fibration. Since $f$ is a strict quasi-split fibration, Prop. 8.12 implies that the above diagram in $\mathrm{Lie}_{n} \mathrm{Alg}^{\mathrm{fin}}$ decomposes into

$$
\begin{array}{cc}
\tau_{<m+1} L \stackrel{\left(q_{<m+1}, r\right)}{\cong} \tau_{\leq m} L \oplus \operatorname{ker} q_{<m+1} \\
\tau_{<m+1} \downarrow & \left|\tau_{\leq m} f \oplus \tau_{<m+1} f\right|_{\mathrm{ker}} \\
\tau_{<m+1} L^{\prime} \stackrel{\left(q_{<m+1}^{\prime}, r^{\prime}\right)}{\cong} \tau_{\leq m} L^{\prime} \oplus \operatorname{ker} q_{<m+1}^{\prime}
\end{array}
$$


Recall from (8.5) that $\operatorname{ker} q_{<m+1}=\operatorname{im} d_{m+1}[-1] \oplus \operatorname{im} d_{m+1}$ and $\operatorname{ker} q_{<m+1}^{\prime}=$ $\operatorname{im} d_{m+1}^{\prime}[-1] \oplus \operatorname{im} d_{m+1}^{\prime}$ are abelian Lie $n$-algebras with isomorphisms as differentials. The $L_{\infty}$-morphism $f$ is surjective in all degrees, and hence $\left.f\right|_{\operatorname{im} d_{m+1}}$ is surjective as well. We obtain the following isomorphisms directly from the definition of the integration functor:

$$
\int \operatorname{ker} q_{<m+1} \cong \operatorname{im} d_{m+1} \otimes \Omega^{m+1}\left(\Delta^{\bullet}\right), \quad \int \operatorname{ker} q_{<m+1}^{\prime} \cong \operatorname{im} d_{m+1}^{\prime} \otimes \Omega^{m+1}\left(\Delta^{\bullet}\right) .
$$

Hence, $\left.\int \tau_{<m+1} f\right|_{\mathrm{ker}}=\left.f\right|_{\mathrm{im} d_{m+1}} \otimes \mathrm{id}_{\Omega}$ is a surjective linear map between simplicial Banach spaces. Therefore from Lemma 5.9 in [17] we deduce that $\left.\int \tau_{<m+1} f\right|_{\text {ker }}$ is a fibration between Lie $\infty$-groups. This observation, when combined with the induction hypothesis and Cor. 9.6, implies that

$$
\int \tau_{\leq m} f \times\left.\int \tau_{<m+1} f\right|_{\mathrm{ker}}=\int\left(\left.\tau_{\leq m} f \oplus \tau_{<m+1} f\right|_{\mathrm{ker}}\right)
$$

is also a fibration. It then follows from the commutativity of diagram (9.11) that $\int \tau_{<m+1} f$ is also a fibration.

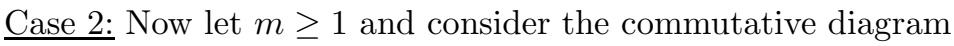

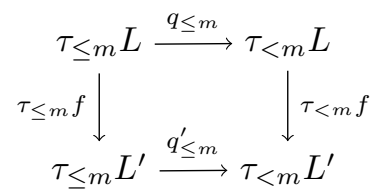

in $\mathrm{Lie}_{n} \mathrm{Alg}^{\text {fin }}$. Suppose that the morphism $\int \tau_{<m} f$ is a fibration in $\mathrm{Lie}_{\infty} \mathrm{Grp}$. We will show that $\int \tau_{\leq m} f$ is also a fibration. Proposition 8.13 gives us the following diagram of strict $L_{\infty}$-morphisms

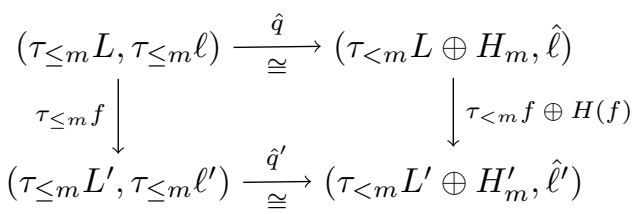

It follows from the definitions of $\hat{q}$ and $\hat{\ell}$ provided in Remarks 8.14 and 8.15, respectively, that the linear projections

$$
\begin{aligned}
\operatorname{pr}_{\tau_{<m} L} & =q_{\leq m} \circ \hat{q}^{-1}:\left(\tau_{<m} L \oplus H_{m}, \hat{\ell}\right) \rightarrow\left(\tau_{<m} L, \tau_{<m} \ell\right) \\
\operatorname{pr}_{\tau_{<m} L^{\prime}} & =q_{\leq m}^{\prime} \circ \hat{q}^{\prime-1}:\left(\tau_{<m} L^{\prime} \oplus H_{m}^{\prime}, \hat{\ell}^{\prime}\right) \rightarrow\left(\tau_{<m} L^{\prime}, \tau_{<m} \ell^{\prime}\right)
\end{aligned}
$$

are strict $L_{\infty}$-morphisms.

We will now show that $\int \tau_{<m} f \oplus H(f)$ is a fibration by applying results based on the discussion following Prop. 9.7 above. Let $k>0$ and $0 \leq j \leq k$. For the sake of brevity, let

$$
\begin{array}{rlrl}
X & :=\int \tau_{<m} L \oplus H_{m}, & X^{\prime}:=\int \tau_{<m} L^{\prime} \oplus H_{m}^{\prime} \\
Y:=\int \tau_{<m} L, & Y^{\prime}:=\int \tau_{<m} L^{\prime} .
\end{array}
$$

Via Cor. 9.9, we tacitly make the following identifications:

$$
\begin{aligned}
X_{k} & =\left\{(\sigma, \rho) \in Y_{k} \times\left(H_{m} \otimes \Omega^{m+1}\left(\Delta^{k}\right)\right) \mid d_{\mathrm{dR}} \rho=\gamma(\sigma)\right\} \\
X\left(\Lambda_{j}^{k}\right) & =\left\{(\eta, \mu) \in Y\left(\Lambda_{j}^{k}\right) \times\left(H_{m} \otimes \Omega^{m+1}\left(\Lambda_{j}^{k}\right)\right) \mid d_{\mathrm{dR}} \mu=\gamma(\eta)\right\},
\end{aligned}
$$


and the analogous identifications for $X_{k}^{\prime}$ and $X^{\prime}\left(\Lambda_{j}^{k}\right)$. Furthermore, in what follows, we adopt the notation used in the commutative diagram (9.10) in Cor. 9.9. For example, we identify $\tau_{<m} f_{*}^{\Omega} \oplus H(f) \otimes \operatorname{id}_{\Omega}$ with the map

$$
\tau_{<m} f \otimes \operatorname{id}_{\Omega} \times H(f) \otimes \operatorname{id}_{\Omega}: X_{k} \rightarrow X_{k}^{\prime} .
$$

Let $\jmath: \Lambda_{j}^{k} \hookrightarrow \Delta^{k}$ denote the inclusion. We wish to show that the map

$$
\left(\tau_{<m} f \otimes \operatorname{id}_{\Omega} \times H(f) \otimes \operatorname{id}_{\Omega}, j^{*}\right): X_{k} \rightarrow X_{k}^{\prime} \times_{X^{\prime}\left(\Lambda_{j}^{k}\right)} X\left(\Lambda_{j}^{k}\right)
$$

is a cover, i.e. a surjective submersion. Note that the pullback on the right hand side above is indeed a manifold since the projection $X_{k}^{\prime} \rightarrow X^{\prime}\left(\Lambda_{j}^{k}\right)$ is a cover. By hypothesis, $\int \tau_{<m} f$ is a fibration hence the usual map

$$
Y_{k} \rightarrow Y_{k}^{\prime} \times_{Y^{\prime}\left(\Lambda_{j}^{k}\right)} Y\left(\Lambda_{j}^{k}\right)
$$

is a cover. The integrations of the $L_{\infty}$-morphisms $\operatorname{pr}_{\tau_{<m} L}$ and $\mathrm{pr}_{\tau_{<m} L^{\prime}}$ from Eq. 9.14 induce a map $X_{k}^{\prime} \times_{X^{\prime}\left(\Lambda_{j}^{k}\right)} X\left(\Lambda_{j}^{k}\right) \rightarrow Y_{k}^{\prime} \times_{Y^{\prime}\left(\Lambda_{j}^{k}\right)} Y\left(\Lambda_{j}^{k}\right)$. Let $Z_{1}$ denote the following pullback

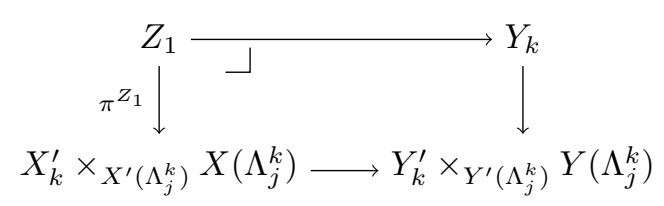

Since the vertical map on the right hand side above is a cover, so is $\pi^{Z_{1}}$. Hence, $Z_{1}$ is a manifold. Note that an element of $Z_{1}$

$$
\left(\left(\sigma^{\prime}, \rho^{\prime}\right),(\eta, \mu), \theta\right) \in Z_{1}
$$

consists of:

- a pair $\left(\sigma^{\prime}, \rho^{\prime}\right) \in \operatorname{MC}\left(\tau_{<m} L^{\prime} \otimes \Omega\left(\Delta^{k}\right)\right) \times\left(H_{m}^{\prime} \otimes \Omega^{m+1}\left(\Delta^{k}\right)\right)$,

- a pair $(\eta, \mu) \in \operatorname{MC}\left(\tau_{<m} L \otimes \Omega\left(\Lambda_{j}^{k}\right)\right) \times\left(H_{m} \otimes \Omega^{m+1}\left(\Lambda_{j}^{k}\right)\right)$, and

- a Maurer-Cartan element $\theta \in \operatorname{MC}\left(\tau_{<m} L \otimes \Omega\left(\Delta^{k}\right)\right)$

such that the following equations hold:

$$
\begin{array}{ll}
d_{\mathrm{dR}} \mu=\gamma(\eta) & d_{\mathrm{dR}} \rho^{\prime}=\gamma^{\prime}\left(\sigma^{\prime}\right) \\
\jmath^{*} \sigma^{\prime}=\left(\tau_{<m} f \otimes \operatorname{id}_{\Omega}\right)(\eta) & \jmath^{*} \rho^{\prime}=\left(H(f) \otimes \operatorname{id}_{\Omega}\right)(\mu) \\
\left(\tau_{<m} f \otimes \operatorname{id}_{\Omega}\right)(\theta)=\sigma^{\prime} & \jmath^{*} \theta=\eta .
\end{array}
$$

Next, we recall that Prop. 9.7 implies that the integration of the $L_{\infty}$-morphism $q_{\leq m}$ in diagram (9.12) is a fibration. It then follows from Eq. 9.14 that

$$
\int \operatorname{pr}_{\tau<m L}: X \rightarrow Y
$$

is also a fibration, and hence the induced map

$$
X_{k} \rightarrow Y_{k} \times_{Y\left(\Lambda_{j}^{k}\right)} X\left(\Lambda_{j}^{k}\right)
$$

is a cover. Equation 9.19 implies that the projection $Z_{1} \rightarrow X\left(\Lambda_{j}^{k}\right)$ and the vertical map $Z_{1} \rightarrow Y_{k}$ in (9.16) induce a map $Z_{1} \rightarrow Y_{k} \times_{Y\left(\Lambda_{j}^{k}\right)} X\left(\Lambda_{j}^{k}\right)$. Let $Z_{2}$ denote the 
following pullback

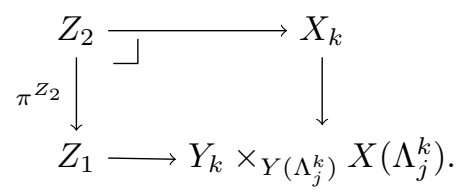

As in the previous case, it follows that $\pi^{Z_{2}}$ is a cover. An element of $Z_{2}$

$$
\left(\left(\sigma^{\prime}, \rho^{\prime}\right),(\eta, \mu),\left(\theta, \theta_{H}\right)\right) \in Z_{2}
$$

consists of an element $\left(\left(\sigma^{\prime}, \rho^{\prime}\right),(\eta, \mu), \theta\right) \in Z_{1}$ and a $(m+1)$-form $\theta_{H} \in H_{m} \otimes$ $\Omega^{m+1}\left(\Delta^{k}\right)$ such that the following equations hold:

$$
d_{\mathrm{dR}} \theta_{H}=\gamma(\theta), \quad \jmath^{*} \theta_{H}=\mu .
$$

In general, we might not have $\left(H(f) \otimes \operatorname{id}_{\Omega}\right)\left(\theta_{H}\right)=\rho^{\prime}$. However, it follows from the definition of the map (9.15) that

$$
d_{\mathrm{dR}}\left(H(f) \otimes \operatorname{id}_{\Omega}\right)\left(\theta_{H}\right)=\gamma^{\prime}\left(\left(\tau_{<m} f \otimes \operatorname{id}_{\Omega}\right) \theta\right) .
$$

Combining this with Eqs. 9.17-9.19 and Eq. 9.21, we deduce that

$$
d_{\mathrm{dR}}\left(H(f) \otimes \mathrm{id}_{\Omega}\right)\left(\theta_{H}\right)=\gamma^{\prime}\left(\sigma^{\prime}\right)=d_{\mathrm{dR}} \rho^{\prime} .
$$

Hence, we have a smooth map

$$
\phi: Z_{2} \rightarrow H_{m}^{\prime} \otimes \Omega_{\mathrm{cl}}^{m+1}\left(\Delta^{k}\right), \quad \phi\left(\left(\sigma^{\prime}, \rho^{\prime}\right),(\eta, \mu),\left(\theta, \theta_{H}\right)\right):=\rho^{\prime}-\left(H(f) \otimes \operatorname{id}_{\Omega}\right)\left(\theta_{H}\right),
$$

where $\Omega_{\mathrm{cl}}^{m+1}\left(\Delta^{k}\right)$ denotes the Banach space of closed $(m+1)$-forms on $\Delta^{k}$.

By hypothesis, $H(f): H_{m} \rightarrow H_{m}^{\prime}$ is surjective. Since $H_{m}$ and $H_{m}^{\prime}$ are finite dimensional, it follows from Lemma 5.9 in [17] that

$$
H(f) \otimes \operatorname{id}_{\Omega}: H_{m} \otimes \Omega_{\mathrm{cl}}\left(\Delta^{\bullet}\right) \rightarrow H_{m}^{\prime} \otimes \Omega_{\mathrm{cl}}\left(\Delta^{\bullet}\right)
$$

is a fibration between simplicial Banach spaces. Hence, the obvious map

$$
H_{m} \otimes \Omega_{\mathrm{cl}}\left(\Delta^{k}\right) \rightarrow H_{m}^{\prime} \otimes \Omega_{\mathrm{cl}}\left(\Delta^{k}\right) \times_{H_{m}^{\prime} \otimes \Omega_{\mathrm{cl}}\left(\Lambda_{j}^{k}\right)} H_{m} \otimes \Omega_{\mathrm{cl}}\left(\Lambda_{j}^{k}\right)
$$

is a cover. Furthermore, since $\jmath^{*}\left(H(f) \otimes \operatorname{id}_{\Omega}\right)\left(\theta_{H}\right)-\jmath^{*} \rho^{\prime}=0$, the map $\phi: Z_{2} \rightarrow$ $H_{m}^{\prime} \otimes \Omega_{\mathrm{cl}}^{m+1}\left(\Delta^{k}\right)$ together with the constant function $Z_{2} \stackrel{0}{\rightarrow} H_{m}^{\prime} \otimes \Omega_{\mathrm{cl}}^{m+1}\left(\Lambda_{j}^{k}\right)$ induce a map from $Z_{2}$ into the pullback appearing in (9.22).

Finally, let $Z_{3}$ denote the following pullback

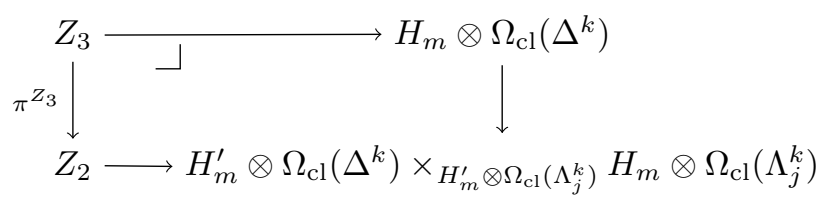

It follows that $Z_{3}$ is a manifold and that $\pi^{Z_{3}}$ is a cover. An element of $Z_{3}$

$$
\left(\left(\sigma^{\prime}, \rho^{\prime}\right),(\eta, \mu),\left(\theta, \theta_{H}\right), \rho\right) \in Z_{3}
$$

consists of an element $\left(\left(\sigma^{\prime}, \rho^{\prime}\right),(\eta, \mu),\left(\theta, \theta_{H}\right)\right) \in Z_{2}$ and a closed $(m+1)$-form $\rho \in H_{m} \otimes \Omega_{\mathrm{cl}}^{m+1}\left(\Delta^{k}\right)$ such that

$$
\left(H(f) \otimes \operatorname{id}_{\Omega}\right)(\rho)=\rho^{\prime}-\left(H(f) \otimes \operatorname{id}_{\Omega}\right)\left(\theta_{H}\right), \quad \jmath^{*} \rho=0 .
$$


Thus we have a smooth map $\chi: Z_{3} \rightarrow X_{k}$ :

$$
\chi\left(\left(\sigma^{\prime}, \rho^{\prime}\right),(\eta, \mu),\left(\theta, \theta_{H}\right), \rho\right):=\left(\theta, \theta_{H}+\rho\right)
$$

that fits into the following commutative diagram:

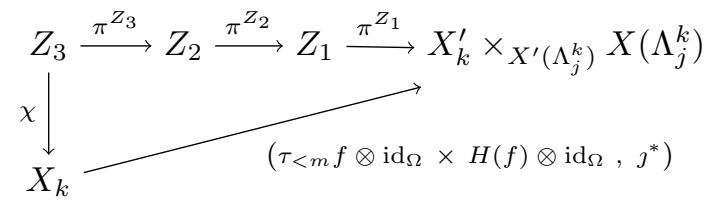

Note that $\chi$ is surjective. Indeed, if $\left(\theta, \theta_{H}\right) \in X_{k}$ then set:

$$
\begin{aligned}
\left(\sigma^{\prime}, \rho^{\prime}\right) & =\left(\tau_{<m} f \otimes \operatorname{id}_{\Omega} \times H(f) \otimes \operatorname{id}_{\Omega}\right)\left(\theta, \theta_{H}\right), \\
(\eta, \mu) & =\jmath^{*}\left(\theta, \theta_{H}\right), \\
\rho & =0,
\end{aligned}
$$

in order to obtain a pre-image of $\left(\theta, \theta_{H}\right)$. Furthermore, the composition

$$
\left(\tau_{<m} f \otimes \operatorname{id}_{\Omega} \times H(f) \otimes \operatorname{id}_{\Omega}, \jmath^{*}\right) \circ \chi
$$

is a surjective submersion, by construction. It then follows from Lemma 6.8 that $\left(\tau_{<m} f \otimes \operatorname{id}_{\Omega} \times H(f) \otimes \operatorname{id}_{\Omega}, J^{*}\right)$ is a surjective submersion. This concludes the proof of the theorem.

Remark 9.11. Not every fibration in $\operatorname{Lie}_{n} \mathrm{Alg}^{\mathrm{fin}}$ integrates to a fibration of Lie $\infty$ groups. If $f: \mathfrak{h} \rightarrow \mathfrak{g}$ is a Lie algebra morphism, then $f$ is a fibration between Lie 1 -algebras, since it is trivially surjective in all positive degrees. Since $\left(\int \mathfrak{h}\right)_{0}=$ $\left(\int \mathfrak{g}\right)_{0}=*$, the integrated map $\int f: \int \mathfrak{h} \rightarrow \int \mathfrak{g}$ satisfies the Kan condition $\operatorname{Kan}(1,1)$ if and only if $\left(\int f\right)_{1}:\left(\int \mathfrak{h}\right)_{1} \rightarrow\left(\int \mathfrak{g}\right)_{1}$ is a surjective submersion. Let $H$ and $G$ be the 1-connected Lie groups integrating $\mathfrak{h}$ and $\mathfrak{g}$, respectively. From Example 9.3 , it follows that $\left(\int f\right)_{1}$ is a surjective submersion if and only if the induced map $f^{I}: P_{e} H \rightarrow P_{e} G$ between based path spaces is a surjective submersion. So if, for example, $f: \mathfrak{h} \rightarrow \mathfrak{g}$ is the inclusion of a proper Lie subalgebra $\mathfrak{h}$ into $\mathfrak{g}$, then $\int f$ is not a fibration.

On the other hand, we presently do not have an example which demonstrates that the identification $H_{0}(L) \cong \operatorname{ker} H(f) \oplus H_{0}\left(L^{\prime}\right)$ is necessary in order for $f$ to be an integrable fibration. Although we do use this property of quasi-split fibrations when we establish the base case in the proof of Thm. 9.10, it might be possible that the integration functor is exact with respect to a larger class of surjective fibrations.

9.3. Integrating weak equivalences. Next, we show that weak equivalences in $\mathrm{Lie}_{n} \mathrm{Alg}{ }^{\text {fin }}$, i.e. $L_{\infty}$-quasi-isomorphisms, between finite type $L_{\infty}$-algebras integrate to weak equivalences in $\mathrm{Lie}_{\infty} \mathrm{Grp}$. Our proof is based on the following result of Henriques, which involves the simplicial homotopy groups $\pi_{*}^{\text {spl }}(X)$ of a $k$-group $X$ defined in Def. 5.2.

Proposition 9.12 (Thm. $6.4[17])$. Let $(L, \ell) \in \mathrm{Lie}_{n} \mathrm{Alg}^{\mathrm{fin}}$, and let $G$ be the 1connected Lie group integrating the Lie algebra $H_{0}(L)$. Then $\pi_{1}^{\mathrm{spl}}\left(\int L\right) \cong G$, and there is a long exact sequence of (sheaves of) groups

$$
\begin{array}{r}
\cdots \rightarrow \pi_{n+1}^{\mathrm{spl}}\left(\int L\right) \rightarrow \pi_{n+1}(G, e) \rightarrow H_{n-1}(L) \rightarrow \pi_{n}^{\mathrm{spl}}\left(\int L\right) \rightarrow \pi_{n}(G, e) \rightarrow \cdots \\
\cdots \rightarrow \pi_{3}^{\mathrm{spl}}\left(\int L\right) \rightarrow \pi_{3}(G, e) \rightarrow H_{1}(L) \rightarrow \pi_{2}^{\mathrm{spl}}\left(\int L\right) \rightarrow \pi_{2}(G, e) .
\end{array}
$$


The long exact sequence (9.24) is functorial with respect to morphisms of Lie $n$-algebras. Although this is not shown in [17], it does follow from the arguments made there, as we now explain.

The sequence (9.24) can be constructed by applying the integration functor of Prop/Def. 9.1 to the Postnikov tower (8.4) of the Lie $n$-algebra $L$. This gives a tower of Kan fibrations between Lie $\infty$-groups [17, Thm. 5.10]. As discussed in the remarks preceding Cor. 6.5 in [17], the spectral sequence associated to this tower is

$$
E_{m, k}^{1}=\pi_{m}^{\mathrm{spl}}\left(\int H_{k-1}(L)[k-1]\right) \Rightarrow \pi_{m+k}^{\mathrm{spl}}\left(\int L\right)
$$

where $H_{k-1}(L)[k-1]$ is the Lie $n$-algebra with only $H_{k-1}(L)$ in degree $k-1$. Prop. 8.10 implies that the spectral sequence is functorial. The spectral sequence is also sparse. As a result, it reduces to a long exact sequence, which becomes (9.24) after identifications are made between certain simplicial homotopy groups, and the Lie group $G$ and its homotopy groups. Therefore, to verify the functoriality of (9.24), it remains to check the naturality of these identifications.

Lemma 9.13. Let $f:(L, \ell) \rightarrow\left(L^{\prime}, \ell^{\prime}\right)$ be a morphism in $\operatorname{Lie}_{n} \mathrm{Alg}^{\text {fin }}$. Let $\Phi: G \rightarrow G^{\prime}$ be the unique homomorphism of 1-connected Lie groups integrating the Lie algebra morphism $\tau_{\leq 0}(f)=\tau_{\leq 0} L \rightarrow \tau_{\leq 0} L^{\prime}$. Then the following diagram commutes

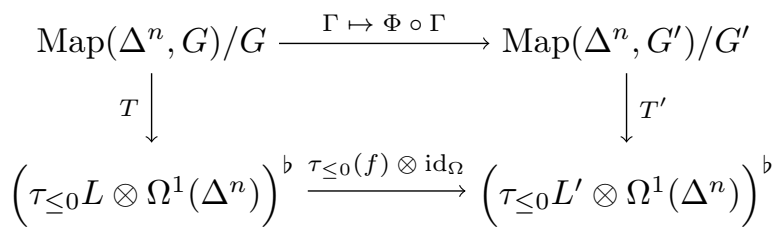

where $T$ and $T^{\prime}$ are the bijections defined in Example 9.2.

Proof. The commutativity of the diagram is verified by using the following elementary facts: (1) $\Phi$, being a homomorphism, intertwines the left multiplication on $G$ with that of $G^{\prime}$, and (2) the differential of $\Phi$ at the identity is $\tau_{\leq 0}(f)$.

Remark 9.14. We note that Lemma 9.13 also implies that the identifications made in Example 6.2 of [17]:

$$
\pi_{1}^{\mathrm{spl}}\left(\int H_{0}(L)\right) \cong G, \quad \pi_{k \geq 2}^{\mathrm{spl}}\left(\int H_{0}(L)\right) \cong \pi_{k \geq 2}(G, e) .
$$

are also natural in $(L, \ell)$.

Now that the functoriality of the long exact sequence (9.24) has been clarified, we can prove the following:

Theorem 9.15. If $f:(L, \ell) \rightarrow\left(L^{\prime}, \ell^{\prime}\right)$ is a weak equivalence in $\mathrm{Lie}_{n} \mathrm{Alg}{ }^{\text {fin }}$, then the morphism

$$
\int f: \int L \rightarrow \int L^{\prime}
$$

is a weak equivalence of Lie $\infty$-groups.

Proof. It is sufficient to show that $\int f$ induces an isomorphism of simplicial homotopy groups, i.e. the induced morphisms of group sheaves

$$
\psi_{m}:=\pi_{m}^{\mathrm{spl}}\left(\int f\right): \pi_{m}^{\mathrm{spl}}\left(\int L\right) \rightarrow \pi_{m}^{\mathrm{spl}}\left(\int L^{\prime}\right) \quad m>0
$$


are isomorphisms. Indeed, if that is the case, then Prop. 5.3 implies that $\int f$ is a stalkwise weak equivalence.

Let $G$ and $G^{\prime}$ denote the simply connected Lie groups integrating the Lie algebras $H_{0}(L)$ and $H_{0}\left(L^{\prime}\right)$, respectively. From Remark 8.1, it follows that

$$
H_{0}\left(f_{1}\right): H_{0}(L) \stackrel{\cong}{\rightarrow} H_{0}\left(L^{\prime}\right)
$$

is an isomorphism of Lie algebras. Let $\Phi: G \stackrel{\cong}{\rightarrow} G^{\prime}$ denote the corresponding isomorphism of Lie groups induced by $H_{0}\left(f_{1}\right)$. It follows from Lemma 9.13 and Remark 9.14 that we have a commuting diagram

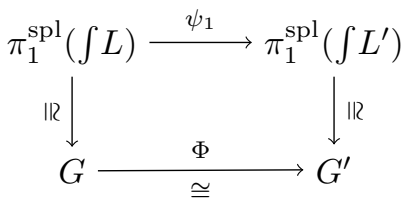

Hence, $\psi_{m}$ is an isomorphism for $m=1$.

The functoriality of the long exact sequence (9.24) gives the commutative diagram (with exact rows)

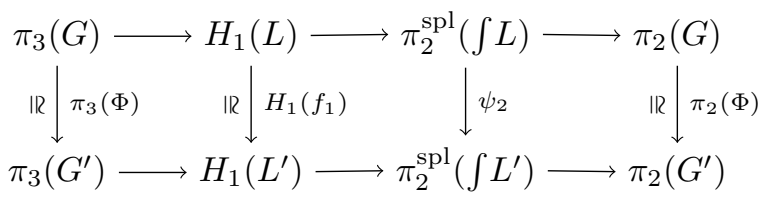

Since $\pi_{2}(G)=\pi_{2}\left(G^{\prime}\right)=0$, a simple diagram chase shows that $\psi_{2}: \pi_{2}^{\mathrm{spl}}\left(\int L\right) \rightarrow$ $\pi_{2}^{\mathrm{spl}}\left(\int L^{\prime}\right)$ is an isomorphism. For $m>2$, we can apply the 5 -lemma to

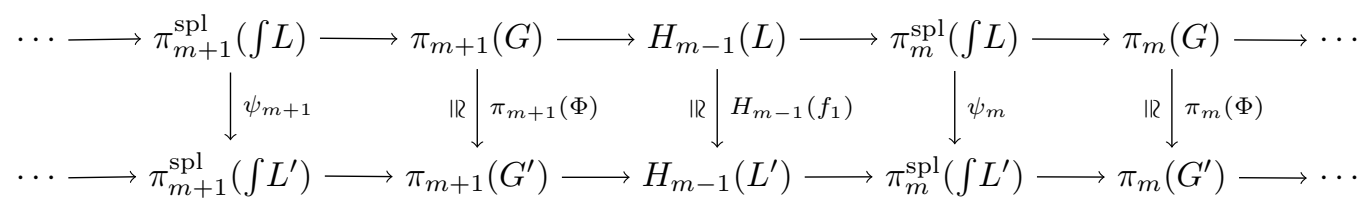

and deduce that $\psi_{m}$ is an isomorphism. This completes the proof.

9.4. The exactness of integration. Let $F: C \rightarrow C^{\prime}$ be a functor between iCFOs. We recall from Def. 2.7 and Def. 2.8 the notion of $F$ being exact with respect to a class of distinguished fibrations $S$ in C. By definition, the class $S$ is required to contain all acyclic fibrations and all morphisms into the terminal object. The functor $F$ is required to preserve the terminal object and acyclic fibrations, and for all $f \in S, F(f)$ must be a fibration in $C^{\prime}$. Finally $F$ is required to preserve pullbacks of fibrations in $S$ along arbitrary morphisms in C.

Note that it follows immediately from Def. 8.6 that the class of quasi-split fibrations in $\mathrm{Lie}_{n} \mathrm{Alg}^{\mathrm{fin}}$ is distinguished. The second main result of this paper is the following: 


\section{Theorem 9.16.}

(1) The integration functor $\int: \mathrm{Lie}_{n} \mathrm{Alg}^{\mathrm{fin}} \rightarrow \mathrm{Lie}_{\infty}$ Grp preserves finite products, weak equivalences, acyclic fibrations, and sends quasi-split fibrations to Kan fibrations.

(2) Any pullback square in $\mathrm{Lie}_{n} \mathrm{Alg}^{\text {fin }}$ of the form

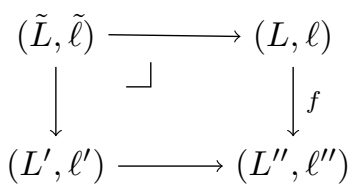

in which $f$ is a quasi-split fibration, is mapped by the integration functor to a pullback square in $\mathrm{Lie}_{\infty}$ Grp.

In particular, the functor $\int: \mathrm{Lie}_{n} \mathrm{Alg}^{\mathrm{fin}} \rightarrow \mathrm{Lie}_{\infty} \mathrm{Grp}$ is exact with respect to the class of quasi-split fibrations.

Proof. It follows from Cor. 9.6 and Thm. 9.10 that the integration functor preserves finite products and sends quasi-split fibrations to Kan fibrations. Theorem 9.15 implies that integration preserves weak equivalences. Therefore, since every acyclic fibration is a quasi-split fibration, it follows that integration preserves acyclic fibrations.

Now suppose we have a pullback diagram of finite type Lie $n$-algebras, as in (9.30), in which $f:(L, \ell) \rightarrow\left(L^{\prime}, \ell^{\prime}\right)$ is a quasi-split fibration. Hence, $\int f$ is a Kan fibration. Since Lie $\infty$-groups are reduced Lie $\infty$-groupoids, the hypotheses of Prop. 7.10 are satisfied and therefore the pullback of the diagram

$$
\int L^{\prime} \stackrel{\int g}{\longrightarrow} \int L^{\prime \prime} \stackrel{\int f}{\longleftarrow} \int L
$$

exists in $\mathrm{Lie}_{\infty}$ Grp. It then follows from Prop. 9.5 that

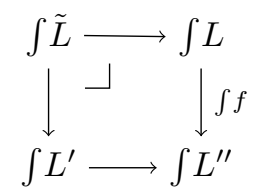

is a pullback diagram of Lie $\infty$-groups.

\section{Appendix A. Proof of Lemma 7.14}

We need to prove the following: For all $n \geq 1$ and $0 \leq j \leq n$, the natural inclusion

$$
\Lambda_{j}^{n} \times \Delta^{1} \sqcup_{\Lambda_{j}^{n} \times \partial \Delta^{1}} \Delta^{n} \times \partial \Delta^{1} \hookrightarrow \Delta^{n} \times \Delta^{1}
$$

is a collapsible extension.

Let us first establish some notation. We fix $n$. Via the usual triangulation, we write $\Delta^{n} \times \Delta^{1}$ as the union $\bigcup_{0 \leq l \leq n} x_{l}$ of $n+1(n+1)$-simplices where:

$$
x_{l}:=\Delta^{n+1}\{(0,0),(1,0), \ldots,(l, 0),(l, 1),(l+1,1), \ldots,(n, 1)\} .
$$


Also, for $0 \leq l \leq n$ and $0 \leq j \leq n$ denote by $y_{l}^{j}$ the following face of the simplex $x_{l}$ :

$$
y_{l}^{j}:= \begin{cases}d_{j+1} x_{l}, & \text { if } 0 \leq l \leq j \\ d_{j} x_{l}, & \text { if } j<l\end{cases}
$$

And for $l=-1, \ldots, n$, and $0 \leq j \leq n$, denote by $T_{j, l} \subseteq \Delta^{n} \times \Delta^{1}$ the following simplicial subsets: If $l=-1$, then

$$
T_{j,-1}:=\Lambda_{j}^{n} \times \Delta^{1} \cup \Delta^{n} \times \partial \Delta^{1}
$$

and for $l \geq 0$

$$
T_{j, l}:=T_{j, l-1} \cup x_{l} .
$$

Note that $T_{j,-1}$ is the left side of the inclusion (A.1), while $T_{j, n}=\Delta^{n} \times \Delta^{1}$. Below, we will prove the lemma by showing the inclusions $T_{j, l-1} \subseteq T_{j, l}$ are collapsible extensions.
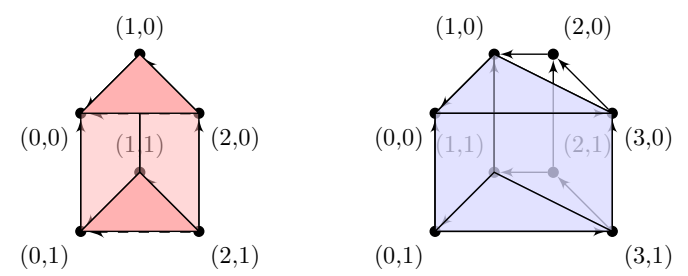

Figure 1. For $n=2$, the left-hand side depicts the geometric realization of the simplicial subset $T_{1,-1} \subseteq \Delta^{2} \times \Delta^{1}$. For $n=3$, the right-hand side depicts the realization of the "face" $d_{2} \Delta^{3} \times \Delta^{1}$ which is missing from $T_{2,-1}$.

We will also need the following simple facts:

Claim A.1. Let $0 \leq j \leq n$ and $0 \leq l \leq n$. Every face of the $(n+1)$-simplex $x_{l}$, with the possible exception of $d_{l+1} x_{l}$ and $y_{l}^{j}$, is contained in the simplicial subset $T_{j, l-1}$.

Proof. We consider the faces $d_{k} x_{l}$. There are three cases. First, if $k=l$, then it is easy to verify that:

$$
\begin{aligned}
& d_{l} x_{l} \subseteq \Delta^{n} \times \partial \Delta^{1} \subseteq T_{j, l-1}, \quad \text { if } l=0 \text { or } l=n \\
& d_{l} x_{l}=d_{l} x_{l-1} \in T_{j, l-1} \quad \text { if } 0<l<n .
\end{aligned}
$$

Now, if $k<l$, then $d_{k} x_{l} \subseteq d_{k} \Delta^{n} \times \Delta^{1}$. Hence, if $k \neq j$, then we have $d_{k} x_{l} \subseteq$ $\Lambda_{j}^{n} \times \Delta^{1} \subseteq T_{j, l-1}$. Otherwise, we have $d_{k} x_{l}=d_{j} x_{l}=y_{l}^{j}$.

Finally, if $k \geq l+2$, then $d_{k} x_{l} \subseteq d_{k-1} \Delta^{n} \times \Delta^{1}$. So if $k \neq j+1$, then $d_{k} x_{l} \subseteq$ $\Lambda_{j}^{n} \times \Delta^{1} \subseteq T_{j, l-1}$, Otherwise, we have $d_{k} x_{l}=d_{j+1} x_{l}=y_{l}^{j}$.

Claim A.2. Let $\Sigma_{j}:=d_{j} \Delta^{n}$ for $0 \leq j \leq n$. Then for $0 \leq i \leq n-1$, we have the inclusion of simplicial sets

$$
d_{i} \Sigma_{j} \times \Delta^{1} \subseteq T_{j,-1} .
$$

Proof. This follows directly from the simplicial identities for face maps. 
Claim A.3. Let $0 \leq j \leq n, 0 \leq l \leq n$, and let $z_{l}^{j}$ denote the $(n-1)$-simplex

$$
z_{l}^{j}:= \begin{cases}d_{l+1} y_{l}^{j}, & \text { if } l \leq j \\ d_{l} y_{l}^{j}, & \text { if } j<l\end{cases}
$$

where $y_{l}^{j}$ is the $n$-simplex (A.2). Every face of $y_{l}^{j}$, with the possible exception of $z_{l}^{j}$, is contained in the simplicial subset $T_{j, l-1}$.

Proof. There are a few cases to consider.

Case $k<l$ : If $0 \leq j \leq l$, then we have $d_{k} y_{l}^{j}=d_{k} d_{j+1} x_{l}=d_{j} d_{k} x_{l}$. Claim A.1 implies that $d_{k} x_{l} \subseteq T_{j, l-1}$, so therefore $d_{k} y_{l}^{j} \subseteq T_{j, l-1}$. If $j<l$, then $d_{k} y_{l}^{j}=d_{k} d_{j} x_{l}$. Hence, we have $d_{k} y_{l}^{j} \subseteq d_{k} \Sigma_{j} \times \Delta^{1}$. So Claim A.2 implies that $d_{k} y_{l}^{j} \subseteq T_{j, l-1}$.

Case $k=l$ : If $j<l$, then $d_{k} y_{l}^{j}=z_{l}^{j}$. If $l \leq j$, then we have $d_{k} y_{l}^{j}=d_{l} d_{j+1} x_{l}=$ $d_{j} d_{l} x_{l}$. Claim A.1 implies that $d_{l} x_{l} \subseteq T_{j, l-1}$, so therefore $d_{k} y_{l}^{j} \subseteq T_{j, l-1}$.

$\underline{\text { Case } k=l+1:}$ If $l \leq j$, then we have $d_{k} y_{l}^{j}=z_{l}^{j}$. If $j<l$, then we have $d_{k} \overline{y_{l}^{j}}=d_{l+1} d_{j} x_{l}=d_{j} d_{l+2} x_{l}$. Claim A.1 implies that $d_{l+2} x_{l} \subseteq T_{j, l-1}$. Hence, $d_{k} y_{l}^{j} \subseteq T_{j, l-1}$.

Case $k \geq l+2$ : If $l \leq j$, then either $d_{k} y_{l}^{j}=d_{j} d_{k} x_{l}$ or $d_{k} y_{l}^{j}=d_{j+1} d_{k+1} x_{l}$, depending on whether $k<j+1$ or $k \geq j+1$. In both cases, Claim A.1 implies that $d_{k} y_{l}^{j} \subseteq T_{j, l-1}$. Finally, if $j<l$, then $d_{k} y_{l}^{j}=d_{k} d_{j} x_{l}$, which implies that $d_{k} y_{l}^{j} \subseteq d_{k} \Sigma_{j} \times \Delta^{1}$. Hence, it follows from Claim A.2 that $d_{k} y_{l}^{j} \subseteq T_{j, l-1}$.

We now arrive at:

Proof of Lemma 7.14. Let $0 \leq j \leq n$ and $0 \leq l \leq n$. We will show that the inclusion $T_{j, l-1} \subseteq T_{j, l}$ is a collapsible extension. First, we observe that the boundary of $x_{l}$ is not contained in $T_{j, l-1}$ : either the face $y_{l}^{j}$ or the face $d_{l+1} x_{l}$ is missing. If $y_{l}^{j}$ is contained in $T_{j, l-1}$, then Claim A.1 implies that there exists a map

$$
\psi: \Lambda_{l+1}^{n+1} \rightarrow T_{j, l-1}
$$

which sends the generators of $\Lambda_{l+1}^{n+1}$ to all the faces of $x_{l}$ except $d_{l+1} x_{l}$. Then the pushout of $\Lambda_{l+1}^{n+1} \hookrightarrow \Delta^{n}$ along $\psi$ is $T_{j, l}$.

On the other hand, if $y_{l}^{j}$ is not contained in $T_{j, l-1}$ then let

$$
k= \begin{cases}l+1 & \text { if } l \leq j \\ l & \text { if } j<l .\end{cases}
$$

We observe that the boundary of $y_{l}^{j}$ is not contained in $T_{j, l-1}$. Claim A.3 implies that there exists a map $\phi: \Lambda_{k}^{n} \rightarrow T_{j, l-1}$ which sends generators of the horn to all faces of $y_{l}^{j}$ except $z_{l}^{j}$. The pushout of $\Lambda_{k}^{n} \hookrightarrow \Delta^{n}$ along $\phi$ gives a simplicial subset $S_{j, l}:=T_{j, l-1} \cup y_{l}^{j}$. If $x_{l}$ is not contained in $S_{j, l}$, then we compose $\psi$ (A.3) with the inclusion $T_{j, l-1} \subseteq S_{j, l}$. The pushout of $\Lambda_{l+1}^{n+1} \hookrightarrow \Delta^{n}$ along this composition is $T_{j, l}$. 


\section{Appendix B. Sheaves on large CATEgories}

As mentioned in Section 3.3.1, there can be set-theoretic technicalities when working with sheaves over large categories. For example, in this paper, we take colimits of representable sheaves (e.g., the simplicial homotopy groups in Def. 5.2) and this implicitly requires a sheafification functor. Unfortunately, the usual plusconstruction for producing a sheaf from a presheaf is not well-defined for large categories, since it a priori requires taking colimits over proper classes. All of this can be avoided by using Grothendieck universes, and in particular the Universe Axiom, which allows us take colimits in an ambient larger universe in which our classes are sets. However, we would like our formalism to not depend on this ambient larger universe in any way. One could have, for example, the colimit of a diagram of representables be a sheaf that takes values in sets which properly reside in the ambient larger universe.

In this appendix, we verify that colimits of representable sheaves are independent of choice of the ambient universe, for those pretopologies on large categories which admit a "small refinement" (Def. B.5). We conclude by showing that our main example of interest: the surjective submersion pretopology $\mathcal{T}_{\text {subm }}$ on the category Mfd of Banach manifolds is a pretopology which admits a small refinement.

We claim no particular originality for these results: they just provide us with an elementary way to comfortably ignore size issues. Our main reference throughout for the set theory involved is Sec. 1.1 of [8], as well as the preprint [27].

\section{B.1. Grothendieck universes.}

Definition B.1. A universe is a set $\mathcal{U}$ that satisfies the following axioms:

(1) If $x \in y$ and $y \in \mathcal{U}$, then $x \in \mathcal{U}$.

(2) If $x$ and $y$ are elements of $\mathcal{U}$, then $\{x, y\} \in \mathcal{U}$.

(3) If $x \in \mathcal{U}$ then the power set $2^{x}$ is an element of $\mathcal{U}$.

(4) If $I \in \mathcal{U}$ and $\left\{x_{\alpha}\right\}_{\alpha \in I}$ is a family of elements of $\mathcal{U}$, then the union $\bigcup_{\alpha \in I} x_{\alpha}$ is an element of $\mathcal{U}$.

(5) The set of all finite von Neumann ordinals is an element of $\mathcal{U}$.

We adopt the following universe axiom:

Assumption B.2 (Universe Axiom). For each set $x$, there exists a universe $\mathcal{U}$ with $x \in \mathcal{U}$.

Any universe $\mathcal{U}$ is a model of ZFC, so all usual set-theoretic constructions apply. A $\mathcal{U}$-set is a member of $\mathcal{U}$, and a $\mathcal{U}$-class is a subset of $\mathcal{U}$. A proper $\mathcal{U}$-class is a $\mathcal{U}$-class which is not a $\mathcal{U}$-set.

Convention B.3. We fix a universe $\mathcal{U}$ in which we consider as the "usual universe" in which we do our mathematics. We denote by Set the category of $\mathcal{U}$-sets.

B.2. Sheaves on locally $\mathcal{U}$-small categories. A category $\mathrm{M}$ is $\mathcal{U}$-small iff $\mathrm{Ob}(\mathrm{M})$ and $\operatorname{Mor}(\mathrm{M})$ are $\mathcal{U}$-sets. We say $\mathrm{M}$ is locally $\mathcal{U}$-small iff $\operatorname{hom}_{\mathrm{M}}(x, y)$ is a $\mathcal{U}$-set for all $x, y \in \mathrm{Ob}(\mathrm{M})$.

B.2.1. Universe extension. Presheaves are only well-defined over categories that are $\mathcal{U}$-small. However, by the universe axiom, there exists a universe $\tilde{\mathcal{U}}$ such that

$$
\mathcal{U} \in \tilde{\mathcal{U}}
$$


It follows from Def. B.1 that $2^{\mathcal{U}}$ is also a $\tilde{\mathcal{U}}$-set, and hence any $\mathcal{U}$-class is as well. We denote by $\widetilde{\text { Set}}$, the category of $\tilde{\mathcal{U}}$-sets. Therefore, if $\mathrm{M}$ is a locally $\mathcal{U}$-small category, then it is $\tilde{\mathcal{U}}$-small category. So, for a well-behaved theory of presheaves on $\mathrm{M}$, we consider the category PSh(M) of $\widetilde{\text { Set-valued functors }}$

$$
F: \mathrm{M}^{\mathrm{op}} \rightarrow \widetilde{\mathrm{Set}} \text {. }
$$

The entire theory of presheaves and sheaves can be applied to those on a locally $\mathcal{U}$-small category without worry of set-theoretic complications, provided we work in $\tilde{\mathcal{U}}$. Let $(\mathrm{M}, \mathcal{T})$ be a locally $\mathcal{U}$-small category equipped with a pretopology (Def. 3.1). Let $\mathcal{T}(-): \operatorname{Ob}(\mathrm{M}) \rightarrow 2^{\operatorname{Mor}(\mathrm{M})}$ denote the function which assigns to an object $X$ the $\tilde{\mathcal{U}}$-set of covers $\mathcal{T}(X)$ of $X$. (Note that $\mathcal{T}(X)$ is a priori not a $\mathcal{U}$-set.)

Let $\operatorname{Sh}(\mathrm{M})$ denote the category of sheaves on $\mathrm{M}$. We have the adjunction

$$
\ell: \mathrm{PSh}(\mathrm{M}) \leftrightarrows \mathrm{Sh}(\mathrm{M}): i
$$

where $i$ is the inclusion, and $\ell$ is the sheafification functor. As usual, the functor $\ell$ preserves finite limits, and the composite $\ell \circ i$ is naturally isomorphic to the identity functor.

B.2.2. Sheafification. Let us quickly describe the sheafification functor $\ell$ for pretopologies in the sense of Def. 3.1. Let $\alpha: U \rightarrow X$ and $\beta: V \rightarrow X$ be a covers of an object $X \in \mathrm{M}$. We say $\alpha$ refines $\beta$, and write $\alpha \prec \beta$ if there exists a morphism $f: U \rightarrow V$ such that $\beta \circ f=\alpha$. The axioms of a pretopology imply that any two covers of an object $X$ have a common refinement, hence the set $\mathcal{T}(X)$ is equipped with a directed preorder.

Let $F$ be a presheaf and $\alpha: U \rightarrow X$ a cover. A matching family for $\alpha$ is an element $x \in F(U)$ such that the following diagram commutes:

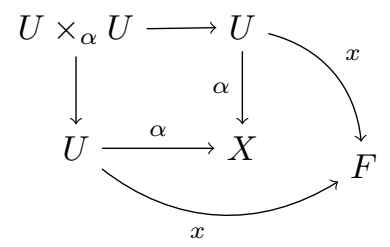

Denote by $\operatorname{Match}(\alpha, F)$ the $\tilde{\mathcal{U}}$ set of all matching families for the cover $\alpha$.

The plus construction applied to a presheaf $F$ produces a new presheaf $F^{+}$, which assigns to an object $X \in \mathrm{M}$, the $\tilde{\mathcal{U}}$-set

$$
F^{+}(X):=\operatorname{colim}_{\alpha \in \mathcal{T}(X)} \operatorname{Match}(\alpha, F) .
$$

An element of $F(X)^{+}$is an equivalence class of matching families $\overline{x_{\alpha}}$. Matching families $x_{\alpha}$ and $x_{\beta}$ for covers $\alpha: U \rightarrow X, \beta: V \rightarrow X$, respectively, are equivalent iff there exists a cover $\gamma: W \rightarrow X$ refining $\alpha$ and $\beta$ such that $x_{\alpha} \circ f=x_{\beta} \circ g$, where $f: W \rightarrow U, g: W \rightarrow V$ are morphisms such that $\gamma=\alpha \circ f=\beta \circ g$. The sheafification functor $\ell: \operatorname{PSh}(M) \rightarrow \operatorname{Sh}(M)$ is then defined as

$$
\ell(F):=\left(F^{+}\right)^{+} .
$$


B.3. Independence of choice of ambient universe. Let $F: \mathrm{M}^{\mathrm{op}} \rightarrow \widetilde{\text { Set }}$ be a presheaf. We say $F$ is a $\mathcal{U}$-presheaf iff $F(X)$ is a $\mathcal{U}$-set for all objects $X \in \mathrm{M}$. The analogous definition for sheaves is clear: A sheaf $F$ is a $\mathcal{U}$-sheaf iff the presheaf $i(F)$ is a $\mathcal{U}$-presheaf. Since $\mathrm{M}$ is locally $\mathcal{U}$-small, the representable sheaves are $\mathcal{U}$-sheaves.

We want $\mathcal{U}$-small colimits and limits involving $\mathcal{U}$-(pre)sheaves to output an object which "stays" in our universe $\mathcal{U}$, and does not depend on the non-canonical choice of the ambient universe $\tilde{\mathcal{U}}$. This is true for $\mathcal{U}$-presheaves, since the inclusion functor

$$
\text { Set } \hookrightarrow \widetilde{\text { Set }}
$$

reflects colimits and limits for all $\mathcal{U}$-small diagrams [27, Cor. 1.19]. And, indeed, this will also be true for $\mathcal{U}$-sheaves, provided we require our pretopology to satisfy a certain smallness condition.

First, we deal with limits of $\mathcal{U}$-sheaves.

Proposition B.4. Let $J$ be aU-small category and $D: J \rightarrow \mathrm{Sh}(\mathrm{M})$ a diagram such that $D(j)$ is a $\mathcal{U}$-sheaf for all objects $j \in J$. Then $\lim D$ is a $\mathcal{U}$-sheaf which can be constructed independently from the choice of ambient universe $\tilde{\mathcal{U}}$.

Proof. Since the inclusion $i: \operatorname{Sh}(C) \rightarrow \operatorname{PSh}(C)$ is a right adjoint, it preserves all $\tilde{\mathcal{U}}$-small limits. Hence, $i \lim D \cong \lim i \circ D$. Now $i \circ D$ is a $\mathcal{U}$-small limit of $\mathcal{U}$ presheaves. Such a limit is computed point-wise, and since Set is complete with respect to $\mathcal{U}$-small limits, it follows that $\lim i \circ D$ is a small presheaf.

\section{B.3.1. A smallness condition for pretopologies.}

Definition B.5. We say that a pretopology $\mathcal{T}$ on a locally $\mathcal{U}$-small category $\mathrm{M}$ admits a $(\mathcal{U}$-)small refinement $\mathcal{O}$ iff for every object $X \in \mathrm{M}$ there is a $\mathcal{U}$-small subset $\mathcal{O}(X) \subseteq \mathcal{T}(X)$ such that every cover in $\mathcal{T}(X)$ is refined by a cover in $\mathcal{O}(X)$.

An example of pretopology which admits a $\mathcal{U}$-small refinement is the surjective submersion topology on the category of Banach manifolds.

Example B.6. Let ( $\left.\mathrm{Mfd}, \mathcal{T}_{\text {subm }}\right)$ denote the category of $(\mathcal{U}$-small) Banach manifolds equipped with the surjective submersion pretopology. Let $M \in \mathrm{Mfd}$. If $\left\{U_{i}\right\}_{i \in I}$ is an open cover of $M$, then the morphism $\coprod_{i \in I} U_{i} \stackrel{\iota}{\rightarrow} M$ is a surjective submersion, where $\iota$ is the unique map induced by the inclusions $U_{i} \subseteq M$. Consider the subset of $\mathcal{T}(M)$

$$
\mathcal{O}(M):=\left\{\coprod_{i \in I} U_{i} \stackrel{\iota}{\rightarrow} M \mid\left\{U_{i}\right\}_{i \in I} \text { is an open cover of } M\right\} .
$$

Since power sets of $\mathcal{U}$-sets are $\mathcal{U}$-sets, $\mathcal{O}(M)$ is a $\mathcal{U}$-set for each $M \in \mathrm{Mfd}$. If $f: N \rightarrow M$ is a surjective submersion, then for each $x \in N$ there exists an open neighborhood $U_{x} \subseteq M$ of $f(x)$ and a map $\sigma_{x}: U_{x} \rightarrow N$ such that $\sigma_{x}(f(x))=x$ and $f \circ \sigma_{x}=\mathrm{id}_{U_{x}}$. Therefore via the universal property of the coproduct, we have a commuting diagram in Mfd

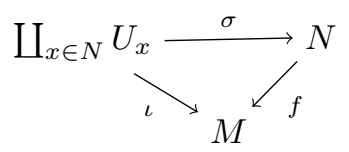

Hence, every cover in $\mathcal{T}_{\text {subm }}(M)$ is refined by a cover in $\mathcal{O}(M)$. 
Note we do not require $\mathcal{O}$ in Def. B.5 to be a pretopology. Nevertheless, taking refinements induces a directed preorder structure on $\mathcal{O}$. If $F$ is a $\mathcal{U}$-presheaf on $(\mathrm{M}, \mathcal{T})$, and $\mathcal{T}$ admits a $\mathcal{U}$-small refinement, then for each $\alpha \in \mathcal{O}(X)$, we have $\operatorname{Match}(\alpha, F) \in$ Set and moreover, we have

$$
\underset{\alpha \in \mathcal{O}(X)}{\operatorname{colim}} \operatorname{Match}(\alpha, F) \in \text { Set }
$$

since this is $\mathcal{U}$-small colimit of $\mathcal{U}$-small sets. Then we have the following:

Theorem B.7. Let $(\mathrm{M}, \mathcal{T})$ be a locally $\mathcal{U}$-small category equipped with a pretopology which admits a $\mathcal{U}$-small refinement. If $F$ is a $\mathcal{U}$-presheaf on $\mathrm{M}$, then its sheafification $\ell(F)$ is a $\mathcal{U}$-sheaf which can be constructed independently from the choice of ambient universe $\tilde{\mathcal{U}}$.

Proof. Let $X \in \mathrm{M}$ and define a new $\mathcal{U}$-presheaf $F_{\mathcal{O}}^{+}$via the colimit

$$
F_{\mathcal{O}}^{+}(X):=\operatorname{colim}_{\alpha \in \mathcal{O}(X)} \operatorname{Match}(\alpha, F) .
$$

There is the obvious function from $F_{\mathcal{O}}^{+}$to the plus construction (B.1) applied to $F$ :

$$
\begin{aligned}
F_{\mathcal{O}}^{+}(X) & \rightarrow F^{+}(X) \\
\overline{x_{\alpha}} & \mapsto \overline{x_{\alpha}},
\end{aligned}
$$

where $\overline{x_{\alpha}}$ on the right hand side above is the equivalence class in $\operatorname{colim}_{\alpha \in \mathcal{T}(X)} \operatorname{Match}(\alpha, F)$ represented by the matching family $x_{\alpha}$. The theorem is proved if this assignment is a bijection. But this follows directly from the fact that for every cover $\alpha \in \mathcal{T}(X)$, there exists a cover in $\mathcal{O}(X)$ refining $\alpha$.

Since the colimit of a diagram of sheaves is constructed by sheafifying the pointwise colimit of the underlying diagram of presheaves, the above theorem gives, as a corollary, the dual of Prop. B.4

Corollary B.8. Let $J$ be a $\mathcal{U}$-small category and $D: J \rightarrow \operatorname{Sh}(\mathrm{M})$ a diagram such that $D(j)$ is a $\mathcal{U}$-sheaf for all objects $j \in J$. Then $\operatorname{colim} D$ is a $\mathcal{U}$-sheaf which can be constructed independently of the choice of ambient universe $\tilde{\mathcal{U}}$.

\section{REFERENCES}

[1] M. P. Allocca, Homomorphisms of $L_{\infty}$ modules, J. Homotopy Relat. Struct. 9 (2014) 285-298.

[2] M. Artin, A. Grothendieck, and J. L. Verdier, Théorie des topos et cohomologie étale des schémas. Tome 2, Lecture Notes in Mathematics, Vol. 270, Springer-Verlag, Berlin, 1972. Séminaire de Géométrie Algébrique du Bois-Marie 19631964 (SGA 4).

[3] K. Behrend and E. Getzler, Geometric higher groupoids and categories. Available as arXiv:1508.02069.

[4] C. Blohmann and C. Zhu, Higher Morita equivalence for $L_{\infty}$ groupoids. In preparation.

[5] K. S. Brown, Abstract homotopy theory and generalized sheaf cohomology, Trans. Amer. Math. Soc. 186 (1973), 419-458.

[6] J. C. Baez and A. S. Crans, Higher-dimensional algebra. VI. Lie 2-algebras, Theory Appl. Categ. 12 (2004), 492-538.

[7] I. Barnea, Y. Harpaz, and G. Horel, Pro-categories in homotopy theory, Algebr. Geom. Topol. 17 (2017), 179-189.

[8] F. Borceux, Handbook of categorical algebra 1: Basic Category Theory. 3, Encyclopedia of Mathematics and its Applications, 50, Cambridge University Press, Cambridge(1994).

[9] V. A. Dolgushev, A. E. Hoffnung and C. L. Rogers, What do homotopy algebras form?, Adv. Math. 274 (2015), 562-605.

[10] E. J. Dubuc, $C^{\infty}$-schemes, Amer. J. Math. 103 (1981), no. 4, 683-690.

[11] D. Dugger, Sheaves and homotopy theory. Available as pages.uoregon.edu/ddugger/cech.html 
[12] J. Duskin, Higher-dimensional torsors and the cohomology of topoi: the abelian theory, in Applications of sheaves (Proc. Res. Sympos. Appl. Sheaf Theory to Logic, Algebra and Anal., Univ. Durham, Durham, 1977), 255-279, Lecture Notes in Math., 753, Springer, Berlin.

[13] W. G. Dwyer and D. M. Kan, Calculating simplicial localizations, J. Pure Appl. Algebra 18 (1980), no. 1, 17-35.

[14] D. S. Freed and M. J. Hopkins, Chern-Weil forms and abstract homotopy theory, Bull. Amer. Math. Soc. (N.S.) 50 (2013), no. 3, 431-468.

[15] E. Getzler, Lie theory for nilpotent $L_{\infty}$-algebras, Ann. of Math. (2) 170, 1 (2009) 271-301;

[16] P. G. Glenn, Realization of cohomology classes in arbitrary exact categories, J. Pure Appl. Algebra 25 (1982), no. 1, 33-105.

[17] A. Henriques, Integrating $L_{\infty}$-algebras, Compos. Math. 144 (2008), no. 4, 1017-1045. arXiv:math/0603563.

[18] G. Horel, Brown categories and bicategories, Homology Homotopy Appl. 18 (2016), 217-232.

[19] M. Hovey, Model categories, Mathematical Surveys and Monographs, 63, Amer. Math. Soc., Providence, RI, 1999.

[20] J. F. Jardine, Simplicial objects in a Grothendieck topos, in Applications of algebraic Ktheory to algebraic geometry and number theory, Part I, II (Boulder, Colo., 1983), 193-239, Contemp. Math., 55, Amer. Math. Soc., Providence, RI.

[21] A. Joyal, Lettre d'André Joyal à Alexandre Grothendieck. Edited by G. Maltsiniotis, 1984. Available at webusers.imj-prg.fr/ georges.maltsiniotis/ps/lettreJoyal.ps

[22] P. T. Johnstone, Sketches of an elephant: a topos theory compendium. Vol. 2, Oxford Logic Guides, 44, Oxford Univ. Press, Oxford, 2002.

[23] B. Jurčo, From simplicial Lie algebras and hypercrossed complexes to differential graded Lie algebras via 1-jets, J. Geom. Phys. 62 (2012), no. 12, 2389-2400. $200 \mathrm{~L}$

[24] T. Lada and M. Markl, Strongly homotopy Lie algebras, Comm. Algebra 23 (1995), no. 6, $2147-2161$.

[25] S. Lang, Differential and Riemannian manifolds, third edition, Graduate Texts in Mathematics, 160, Springer, New York, 1995.

[26] D. Li. Higher Groupoid Actions, Bibundles, and Differentiation. Ph.D. thesis, Georg-August University, Göttingen, 2014. Available as arXiv:1512.04209.

[27] Z. L. Low, Universes for category theory. Available as arXiv:1304.5227v2.

[28] S. Mac Lane and I. Moerdijk, Sheaves in geometry and logic, corrected reprint of the 1992 edition, Universitext, Springer, New York, 1994.

[29] T. Nikolaus, U. Schreiber and D. Stevenson, Principal $\infty$-bundles: presentations, J. Homotopy Relat. Struct. 10 (2015), no. 3, 565-622.

[30] T. Nikolaus and C. Schweigert, Equivariance in higher geometry, Adv. Math. 226 (2011), no. 4, 3367-3408.

[31] B. Noohi, Integrating morphisms of Lie 2-algebras, Compos. Math. 149 (2013), no. 2, 264294.

[32] J. P. Pridham, Presenting higher stacks as simplicial schemes, Adv. Math. 238 (2013), 184245.

[33] D. Quillen. Rational homotopy theory. Ann. of Math. (2), 90:205-295, 1969.

[34] E. Riehl, Categorical homotopy theory, New Mathematical Monographs, 24, Cambridge University Press, Cambridge, 2014.

[35] C. Rogers, Homotopical properties of the simplicial Maurer-Cartan functor. MATRIX Annals, MATRIX Book Series 1 Springer, Berlin, 2018. arXiv:1612.07868.

[36] C. Rogers, An explicit model for the homotopy theory of finite type Lie $n$-algebras. Available as arXiv:1809.05999.

[37] P. Ševera, $L_{\infty}$-algebras as first approximations, in XXVI Workshop on Geometrical Methods in Physics, 199-204, AIP Conf. Proc., 956, Amer. Inst. Phys., Melville, NY. 2007.

[38] P. Ševera and M. Širaň, Integration of differential graded manifolds. Available as arXiv:1506.04898.

[39] B. Vallette, Homotopy theory of homotopy algebras. Available as arXiv:1411.5533.

[40] W. C. Waterhouse, Basically bounded functors and flat sheaves, Pacific J. Math. 57 (1975), no. 2, 597-610.

[41] M. Weiss, Hammock localization in Waldhausen categories, J. Pure Appl. Algebra 138 (1999), 185-195. 
HOMOTOPY THEORY FOR LIE $\infty$-GROUPOIDS \& APPLICATION TO INTEGRATION 71

[42] J. Wolfson, Descent for $n$-bundles, Adv. Math. 288 (2016), 527-575. arXiv:1308.1113.

[43] C. Zhu, n-groupoids and stacky groupoids, Int. Math. Res. Not. 2009, no. 21, 4087-4141. arXiv:0801.2057.

Department of Mathematics and Statistics, University of Nevada, Reno. 1664 N. Virginia Street Reno, NV 89557-0084 USA

E-mail address: chrisrogers@unr.edu, chris.rogers.math@gmail.com

Mathematisches Institut and Courant Research Centre "Higher Order Structures", Georg-August-Universität Göttingen, Bunsenstrasse 3-5, 37073 Göttingen, Germany

E-mail address: zhu@uni-math.gwdg.de, chenchang.zhu@gmail.com 